\title{
Future Science at the Relativistic Heavy Ion Collider
}

December 30, 2006
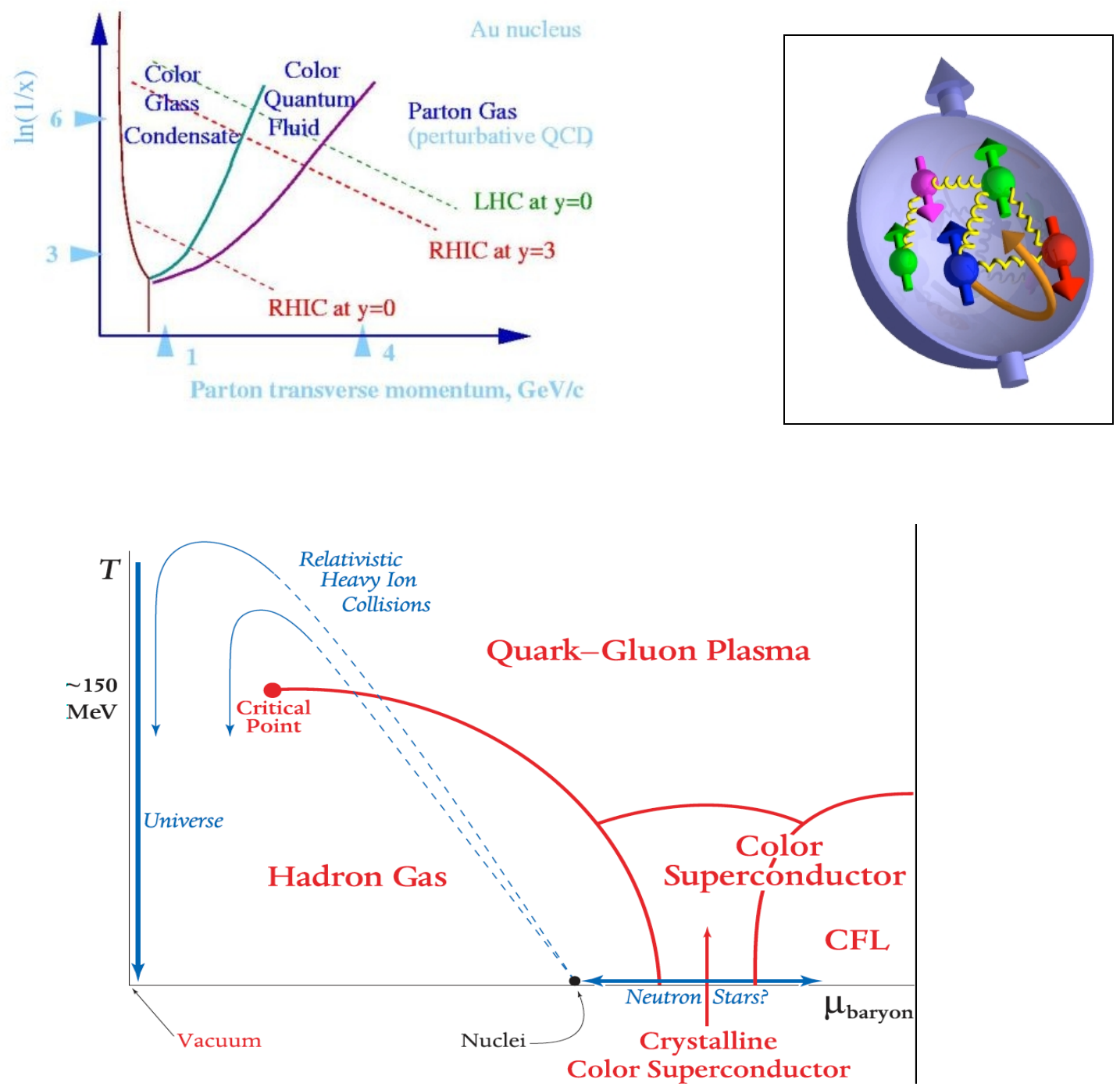

\section{Summary of the $2004-2005$ RHIC II Science Working Groups}

Notice: This manuscript has been authored by employees of Brookhaven Science Associates, LLC under Contract No. DE-AC02-98CH10886 with the U.S. Department of Energy. The publisher by accepting the manuscript for publication acknowledges that the United States Government retains a non-exclusive, paid-up, irrevocable, world-wide license to publish or reproduce the published form of this manuscript, or allow others to do so, for United States Government purposes. 


\section{Table of contents}

1. Overview $\quad 4$

2. Summary of the first 5 years at RHIC 9

2..1. Heavy ion physics 9

2..2. Spin physics 18

3. The RHIC facility - evolution and future $\quad 22$

4. Fundamental questions for the next ten years at RHIC 25

4.1. What are the phases of QCD matter?

4.2. What is the wave function of a heavy nucleus. 26

4.3. What is the wave function of the proton?

4.4. What is the nature of non-equilibrium processes in a fundamental theory? 27

5. The future physics program at RHIC 28

5.1. Equation of state and the QCD phase diagram 29

$\begin{array}{ll}\text { 5.1.1. Dynamical considerations } & 29\end{array}$

$\begin{array}{ll}\text { 5.1.1.1. Evidence for thermalization } & 29\end{array}$

5.1.1.2. Timescale for thermalization 30

5.1.1.3. Thermalization mechanism and links to experiment 30

5.1.1.4. Viscosity 31

5.1.2. Equation of state 34

$\begin{array}{ll}\text { 5.1.2.1. Measurements of energy density } & 34\end{array}$

5.1.2.2. Initial temperature: how to measure and use it? 37

5.1.3. Exploring the QCD phase diagram $\quad 40$

5.1.3.1. Search for the QCD critical point 40

5.1.3.2. Medium effects on properties of hadrons 41

5.1.4 Deconfinement $\quad 42$

5.1.5. Hadronization 48

5.2. Gluon saturation $\quad 50$

5.3. Spin structure of the nucleon $\quad 52$

5.4. Non-equilibrium processes.

6. RHIC II and other future heavy ion facilities $\quad 59$

7. Relationship to other areas $\quad 62$

7.1. Plasma physics $\quad 62$

7.2. Condensed matter physics $\quad 62$

$\begin{array}{ll}\text { 7.3. Cosmology } & 63\end{array}$

7.4. String theory $\quad 63$

$\begin{array}{ll}\text { 8. Summary } & 64\end{array}$

Appendix I: RHIC II science workshops $\quad 66$

Side Bar Summaries:

Exploring heavy ion collisions... the emerging picture 16

How do we know it flows? 17

QCD and the spin of the proton $\quad 21$

Do heavy quarks flow 33

Probing the dense medium with jets: RHIC II and LHC 39

Quarkonium yields at RHIC II and LHC $\quad 46$

$\mathrm{J} / \psi$ flow $\quad 47$ 


\section{Future Science at the Relativistic Heavy Ion Collider}

\section{Overview}

QCD was developed in the 1970's as a theory of the strong interaction describing the confinement of quarks in hadrons. An early consequence of this picture was the realization that at sufficiently high temperature, or energy density, the confining forces are overcome by color screening effects, resulting in a transition from hadronic matter to a new state - later named the Quark Gluon Plasma - whose bulk dynamical properties are determined by the quark and gluon degrees of freedom, rather than those of confined hadrons. The suggestion that this phase transition in a fundamental theory of nature might occur in the hot, dense nuclear matter created in heavy ion collisions triggered a series of experimental searches during the past two decades at CERN and at BNL, with successively higher-energy nuclear collisions. This has culminated in the present RHIC program.

In their first five years of operation, the RHIC experiments have identified a new form of thermalized matter formed in $\mathrm{Au}+\mathrm{Au}$ collisions at energy densities more than 100 times that of a cold atomic nucleus. Measurements and comparison with relativistic hydrodynamic models indicate that the matter thermalizes in an unexpectedly short time $(<1 \mathrm{fm} / \mathrm{c})$, has an energy density at least 15 times larger than needed for color deconfinement, has a temperature about 2 times the critical temperature of $\sim 170 \mathrm{MeV}$ predicted by lattice QCD, and appears to exhibit collective motion with ideal hydrodynamic properties - a "perfect liquid" that appears to flow with a near-zero viscosity to entropy ratio - lower than any previously observed fluid and perhaps close to a universal lower bound.

There are also indications that the new form of matter directly involves quarks. Comparison of measured relative hadron abundances with very successful statistical models indicates that hadrons chemically decouple at a temperature of $160-170 \mathrm{MeV}$. There is evidence suggesting that this happens very close to the quark-hadron phase transition, ie. that hadrons are born in the phase transition from quark matter, and abundance-changing interactions then quickly cease. Valence quark number scaling of the measured anisotropy parameter for all hadrons suggests that the collectively flowing matter involves quarks, not hadrons. And the striking observation of a universal, strong enhancement of baryons relative to mesons at intermediate transverse momentum has been interpreted as evidence of competition between quark coalescence of the bulk medium and jet fragmentation.

It is generally agreed that the new matter is not describable in terms of ordinary color neutral hadrons, and that many observations are consistent with models that incorporate quark and gluon degrees of freedom. The evidence is consistent with the matter being a strongly coupled quark gluon plasma (sQGP), and thus it behaves quite differently from the perturbative QCD parton gas that was expected by most people prior to RHIC data. The extraordinary properties of this new state of matter demand further measurements to better understand its behavior, properties, origin and description.

The RHIC program has also found unexpectedly low multiplicities of produced hadrons in heavy ion collisions and suppression of high transverse momentum particles in the forward direction in $\mathrm{d}+\mathrm{Au}$ collisions, both of which are consistent with gluon saturation in the colliding nuclei playing 
an important role at RHIC energies. The saturation scale represents a fundamental property of hadrons and nuclei, and so it is essential that we determine if gluon saturation is the correct explanation for the existing RHIC data. This will be addressed prior to RHIC II by new capabilities provided by detector upgrades, after which RHIC II luminosities will enable powerful, detailed tests of theory using measurements with rare probes.

Finally, the world's first polarized proton collider has been commissioned at RHIC, and the task of trying to understand the spin structure of the proton has begun. The RHIC spin goals include direct measurements of the gluon and flavor-separated quark and antiquark contributions to the proton's spin, and studies of the transverse spin and transverse motion preferences of the quarks and gluons in a transversely polarized proton. These goals will be met by a series of precision measurements using processes that can be understood well using pQCD. These measurements require very large integrated luminosity, but they have huge potential to resolve the long-standing puzzle of the origin of proton spin in terms of its constituents.

The interaction between theory and experiment has been crucial to the success of the RHIC program, beginning with the prediction of the existence of a quark gluon plasma that could be described by QCD. The rapid succession of important new experimental results from RHIC has overturned many early theoretical ideas, which has in turn led to discoveries that have been the result of an experimental program guided by new theoretical concepts. These include the mechanism of energy loss in a high-density medium of color charges, the consequences of a dense gluonic initial state, and the realization of a strongly-coupled fluid with ideal hydrodynamic properties, to name a few. In addition to these conceptual advances, experimental progress is greatly enhanced by the timely availability of certain theoretical calculations. For example, lattice QCD calculations now provide accurate descriptions of the phase properties of bulk QCD matter at finite temperature, including the equation of state. Also, realistic calculations of jet and heavy quark production, as well as detailed three-dimensional hydrodynamic simulations of heavy ion collisions are becoming an increasingly important tool for the full understanding of experimental data.

The initial years of RHIC operation have resulted in spectacular advances in our understanding of hot nuclear matter. But a fundamental understanding of the medium seen in heavy ion collisions at RHIC does not yet exist. That, and understanding the origin of the proton's spin, will require new data that will, in turn, require enhanced capabilities of the RHIC detectors and accelerator. The main focus of this report is to outline the scientific opportunities for an upgraded RHIC facility RHIC II - in the coming years.

The detector upgrades and the RHIC II luminosity upgrade are designed to address the key scientific questions regarding this newly-discovered form of QCD matter. Examples of these questions are:

\section{What are the properties of the medium.}

- What is the mechanism of the unexpectedly fast thermal equilibration?

RHIC II will enable precise measurements of the production and flow characteristics of heavy quarks (charm and beauty), which are expected to thermalize much more slowly than light partons. These measurements can be used 
to constrain microscopic transport models of the thermalization process.

- What is the initial temperature and thermal evolution of the produced matter? RHIC II will enable photon and di-lepton measurements in kinematic regimes where radiation from the hot QCD matter outshines other sources of EM signals, providing for the first time experimental measures of the temperature as the system evolves.

- What is the energy density and equation of state of the medium?

RHIC II will provide usable statistical samples of $\gamma$-jet coincidences, the "golden probe" for precisely measuring the energy loss of hard scattered partons

propagating through the medium. Existing jet energy loss data provide only a lower limit on the density.

- What is the viscosity of the produced matter?

RHIC II, along with continued progress in theory, will provide detailed comparison of measured collective flow patterns with a viscous hydrodynamic framework. High-statistics flow measurements for many identified hadrons, including multistrange baryons and mesons, charm mesons, and direct photons are needed in several symmetric and asymmetric systems to fully constrain models.

- Is there direct evidence for deconfinement, color screening, and a partonic nature of the hot, dense medium? What is the screening length?

RHIC II will provide high-statistics data on bound states of heavy quark-antiquark pairs (charmonium and bottomonium) - including excited states of the $\mathrm{J} / \psi$ and upsilon - in hot and cold nuclear matter, for comparison with theoretical calculations for melting and regeneration of these states in the presence of strong color fields.

- Is chiral symmetry restored, as predicted by QCD?

The detector upgrades and increased luminosity at all collision energies will make possible a search for medium modification of hadron properties over a range of baryon densities by studying their dilepton decays, to look for evidence of the predicted onset of chiral symmetry restoration.

- How does the new form of matter hadronize at the phase transition? Studying the interplay of recombination and fragmentation will require high precision data on identified particle distributions covering $\mathrm{p}_{\mathrm{T}} \sim 8 \mathrm{GeV} / \mathrm{c}$, with data on rare multi-strange hadrons and resonances being of particular interest. The measurement of dynamical two-particle correlations will be used to study recombination and the transition to jet fragmentation at high $\mathrm{p}_{\mathrm{T}}$. Detector upgrades and RHIC II luminosity will be required.

\section{Can we directly observe a QCD phase transition? Where is the QCD critical point?} By providing increased luminosity at lower energies, RHIC II will enable a systematic search of the QCD phase diagram to locate the QCD critical point by varying the baryochemical potential while looking for signs of proximity to a thermodynamic singularity, such as large event by event fluctuations.

\section{What is the initial state in heavy ion collisions?}

The gluon saturation scale is a fundamental property of hadrons and nuclei that is not yet 
well determined. To separate gluon saturation effects from other effects, multi-particle correlation measurements involving rare probes produced at forward rapidity by hard scattering processes in pA or dA collisions will be needed. This will need detector upgrades and RHIC II.

\section{How does the nucleon get its spin?}

The influence of sea quarks on the properties of the nucleon is still an open question. RHIC will embark on a program to determine the $\bar{u}$ and $\bar{d}$ contributions to the proton spin through measurements of spin asymmetries in $\mathrm{W}$ boson production as soon as detector upgrades are in place. RHIC II will extend these studies to allow high-precision measurements of strange quark and anti-quark spin densities in the proton through asymmetries in the production of $\mathrm{W}$ bosons in coincidence with charm particles.

The planned upgrades to the PHENIX and STAR detectors will allow direct identification of the quark flavors - up, down, strange, charm, beauty - over a wide range of final-state kinematics in RHIC collisions, and will extend the solid-angle coverage for high energy photons, hadrons, and jets. These enhancements will allow PHENIX and STAR to address some of the crucial questions enumerated above with the current capabilities of the RHIC complex. But many of the most important measurements described in this report call for data samples with sensitivity to processes that occur at the level of once per 100 million gold-gold collisions. Such data samples, along with the necessary comparison data, would take several decades to accumulate at present collider luminosities. The goal for RHIC II is to make these measurements achievable by increasing the RHIC heavy ion luminosity by an order of magnitude through electron cooling of the ion beams.

The proposed RHIC II luminosity upgrade will follow the STAR and PHENIX detector upgrades. A detailed plan to implement these upgrades has been developed by BNL in collaboration with the RHIC scientific community. This "Mid-Term Strategic Plan for RHIC" details the scientific program, facility operations, machine and detector R\&D, and capital construction during the years 2006 - 2011. The plan was submitted to DOE in February, 2006, and can be found on the web at http://www.bnl.gov/HENP/docs/RHICplanning/RHIC Mid-termplan_print.pdf.

In the longer term, BNL plans to build on these upgrades to enhance the capabilities for exploring the phenomena observed in the spin structure of nucleons and in new forms of QCD matter with the construction of an electron beam to collide with the cooled ion beams and polarized protons of RHIC II. This facility, eRHIC, will extend the RHIC complex to a full-capability QCD laboratory, with high-luminosity colliding beams in all combinations of polarized electrons, polarized protons, and the full range of ion species. The planning and R\&D for eRHIC is spelled out in the MidTerm Strategic Plan, which envisions the completion of such a facility about ten years from now.

As the RHIC II upgrades come online, the Large Hadron Collider (LHC) at CERN will be completed, accelerating heavy ion beams as heavy as $\mathrm{Pb}$ for one month each year, exploring heavy ion collisions at yet higher energy. The LHC will extend the range of initial temperatures by a factor of $\sim 2$, with the possibility of revealing entirely new phenomena. RHIC II, with its enhanced luminosity, and operation dedicated to QCD studies, will broadly explore the phase diagram with the ability to vary collision energy and beam species multiple times in a single year, while still accumulating large integrated luminosities. Throughout this report we address the 
complementary roles of RHIC II and the LHC in this exciting new era of discovery.

With the advent of RHIC II, the ion beam capability of the LHC, and ultimately eRHIC, the new landscape of QCD matter will be opened for detailed investigation, addressing a range of fundamental scientific questions that bring a new dimension to the role of Nuclear Physics: What are the phases of QCD matter? What is the nature of non-equilibrium processes in the theory of quantum chromodynamics? What are the wave functions of the nucleon, and of heavy nuclei? In this report we begin with a discussion of these questions, and then summarize in quantitative detail the measurements that motivate and define the RHIC II program.

Much of the input to this report comes from a series of community-wide RHIC II Science Workshops, convened in 2004-2005 to examine the questions raised by the RHIC measurements to date, and to provide explicit requirements for an experimental program to survey the new landscape of QCD at high temperature and density, and to investigate the spin structure of the nucleon. These workshops resulted in a set of six Working Group Reports that provide the supporting documentation for this summary. The Working Group Reports may be found at: http://www.bnl.gov/physics/rhicIIscience/ under the headings:

- $\quad$ High $\mathrm{P}_{\mathrm{T}}$

- Heavy Flavor

- Equation of State

- Electromagnetic Probes

- Forward Physics

- Spin

Detailed reviews from the four experiments, published in 2005, of the significance of the RHIC results are also of interest and can be found in:

- "Quark-gluon plasma and color glass condensate at RHIC? The perspective from the BRAHMS experiment", I. Arsene et. al. (The BRAHMS Collaboration), Nuclear Physics A $757(2005) 1$.

- "The PHOBOS perspective on discoveries at RHIC", The PHOBOS Collaboration, Nuclear Physics A 757 (2005) 28.

- "Experimental and theoretical challenges in the search for the quark-gluon plasma: The STAR Collaboration's critical assessment of the evidence from RHIC collisions", The STAR Collaboration, Nuclear Physics A 757 (2005) 102.

- "Formation of dense partonic matter in relativistic nucleus-nucleus collisions at RHIC: Experimental evaluation by the PHENIX Collaboration", The PHENIX Collaboration, Nuclear Physics A 757 (2005) 184.

This document does not contain references to the original literature. The detailed support case for the statements made here is contained in the working group reports, which contain detailed discussions and extensive references to the literature. 


\section{Summary of the first 5 years at RHIC}

In the first five runs (2000-2005), BRAHMS, PHENIX, PHOBOS and STAR have collected data from $\mathrm{Au}+\mathrm{Au}, \mathrm{d}+\mathrm{Au}, \mathrm{Cu}+\mathrm{Cu}$, and $\mathrm{p}+\mathrm{p}$ collisions, with $\mathrm{Au}+\mathrm{Au}$ collisions having been studied at four collision energies $\left(\sqrt{s}_{\mathrm{s}}=19.6,63,130\right.$ and $\left.200 \mathrm{~A} \mathrm{GeV}\right)$. The largest data samples were collected at the highest energy of $\sqrt{\mathrm{s}}_{\mathrm{s}}=200 \mathrm{~A} \mathrm{GeV}$. In addition, a spin physics program with polarized proton beams has been started. The possibility to study proton-proton, proton-nucleus and nucleus-nucleus collisions with identical center-of-mass energies at the same facility has been the key to eliminating many sources of systematic error. Nearly all observables have been studied systematically as a function of collision centrality and of the emission angle relative to the reaction plane, thereby providing complete control over the collision geometry. It is noteworthy that from the very beginning of RHIC operations there has in general been quantitative agreement between the results obtained by the four RHIC experiments wherever their acceptances overlap.

\subsection{Heavy-ion physics}

Results from the first five years of RHIC operations with heavy ions have provided solid evidence for the creation of a new state of thermalized matter at unprecedented energy densities (more than 100 times larger than that of cold atomic nuclei) which appears to exhibit almost perfect fluid dynamical collective behavior, and strong indications that this thermalized matter originated from a high energy density state of gluons with possibly universal properties. Among this evidence four fundamental new discoveries stand out:

- enormous collective motion of the medium, in agreement with near-zero viscosity hydrodynamic behavior (often characterized as "perfect fluid" behavior), pointing to rapid thermalization and strong coupling of the matter [Fig. 1],

- quenching of jets, observed via the suppression of particle production at high transverse momenta and the dramatic modification of jet correlations in central $\mathrm{Au}+\mathrm{Au}$ collisions, pointing towards dramatic energy loss of partons traversing matter of very high color charge density [Fig. 2],

- large, anomalous enhancement of baryon and anti-baryon production rates at intermediate transverse momentum relative to mesons, together with scaling of hadron production yields and their collective motion with the number of valence quarks, suggesting that hadrons form by quark coalescenceafter the flow occurs [Figs. 3, 4],

- First indications of gluon saturation in heavy ion collisions, as evidenced by reduced total particle production at RHIC energies [Fig. 5].

In addition, RHIC experiments confirmed with higher accuracy and better systematics important features of ultra-relativistic heavy-ion collisions that were previously discovered at lower energies. These include

- hadron abundance ratios that are characterized by a chemical equilibrium distribution with "chemical freeze-out" temperature $\mathrm{T}_{\text {chem }} \approx 160-170 \mathrm{MeV}$ [Fig. 6], a value that is observed to be independent of collision system and collision centrality and agrees with the value for the quark-hadron phase transition temperature predicted by Lattice QCD, 


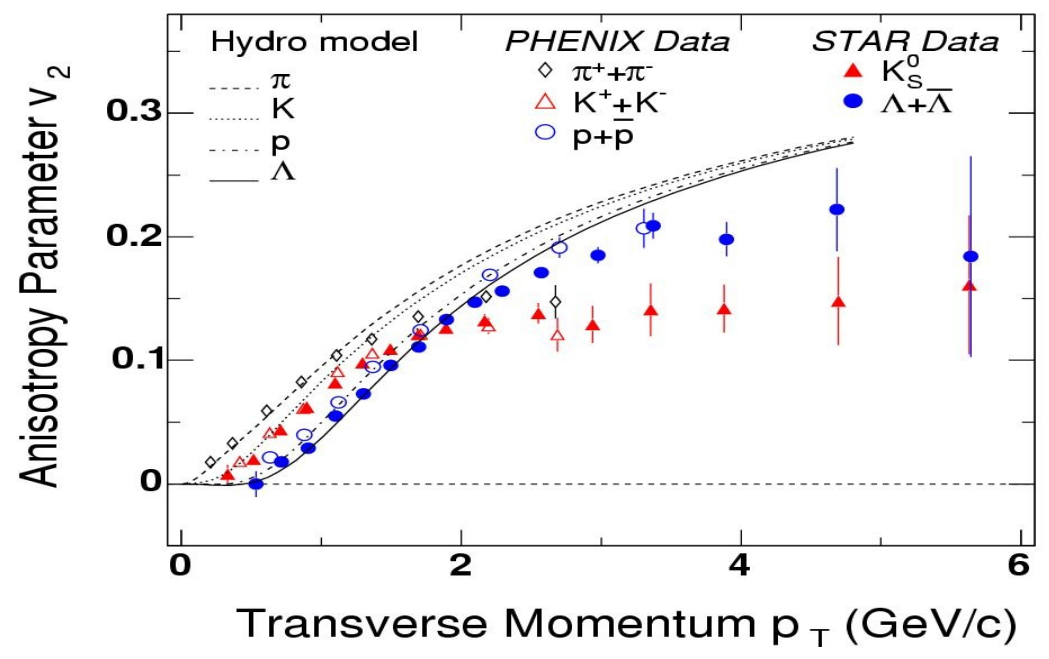

Fig. 1: Elliptic flow $\left(\mathrm{v}_{2}\right)$ plotted as a function of transverse momentum $\left(\mathrm{p}_{\mathrm{T}}\right)$ for identified hadrons.The elliptic flow is a measure of the anisotropic pressure-driven expansion in off-center collisions (see the sidebar on flow for an explanation). Compilation of STAR and PHENIX data compared with hydrodynamic predictions. Note that the bulk of the particle production is at less than $2 \mathrm{GeV} / \mathrm{c}$. Hydro is expected to break down at $\mathrm{p}_{\mathrm{T}}>1.5-2 \mathrm{GeV} / \mathrm{c}$.

- a strong transverse momentum dependence of the Hanbury Brown - Twiss (HBT) radius parameters extracted from two-particle momentum correlations between pairs of identical particles, consistent with predictions from models which incorporate fast collective expansion of the collision fireball at hadronic freeze-out,

- the observation in non-central collisions of an angular variation of these HBT radii with the azimuthal emission angle relative to the reaction plane that is qualitatively consistent with hydrodynamic models for the time-evolution of the spatial eccentricity of the collision fireball in such collisions.

First results on charmonium production and direct photon emission have been obtained, but they only begin to explore the physics to be learned from these probes. An unexpectedly strong suppression of electrons from charmed hadron decays at high transverse momenta has been observed. First studies of elliptic flow of electrons from semileptonic decays of charmed hadrons seem to indicate large flow anisotropies for charm quarks, suggesting strong interactions between even charm quarks and the collectively expanding medium. 

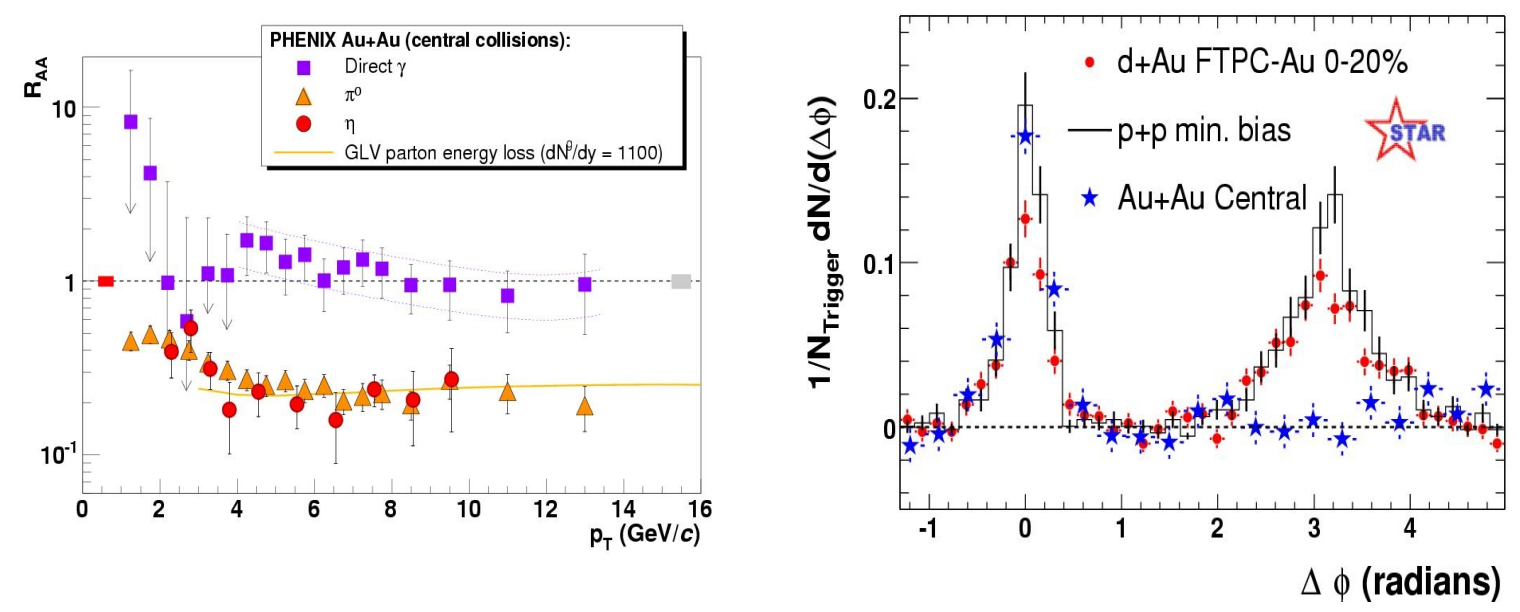

Fig. 2: Left: Nuclear modification factor $\mathrm{R}_{\mathrm{AA}}\left(\mathrm{p}_{\mathrm{T}}\right)$ for $\gamma, \pi^{0}$ and $\eta$ in central $\mathrm{Au}+\mathrm{Au}$ collisions. The nuclear modification factor is the ratio of the cross section per nucleon-nucleon collision measured in a heavy ion collision divided by the cross section measured in pp. If there were no nuclear effects it would be unity. Note the strong suppression of the mesons and the lack of suppression for the $\gamma$, which do not interact with the final state medium. Right: Correlations in azimuthal angle between high momentum hadrons showing evidence of suppression of back-toback jets in central $\mathrm{Au}+\mathrm{Au}$ relative to pp and $\mathrm{dAu}$ collisions.

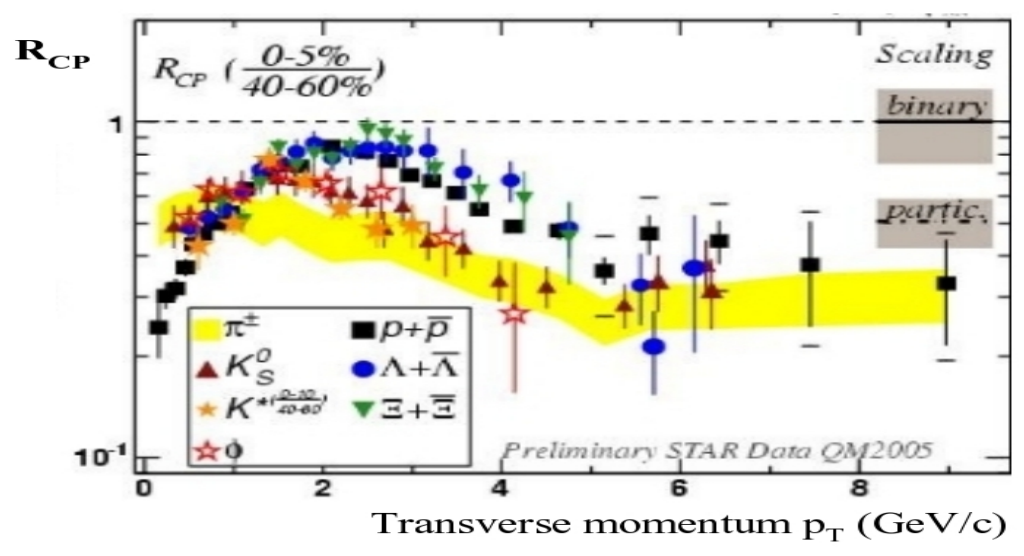

Fig. 3: Nuclear modification factor $\mathrm{R}_{\mathrm{CP}}\left(\mathrm{p}_{\mathrm{T}}\right)$ from STAR and PHENIX for identified hadrons, showing the appearance of two separate bands for baryons and mesons. $R_{C P}$ is the ratio of cross section per nucleon-nucleon collision in central events to that in peripheral events. 


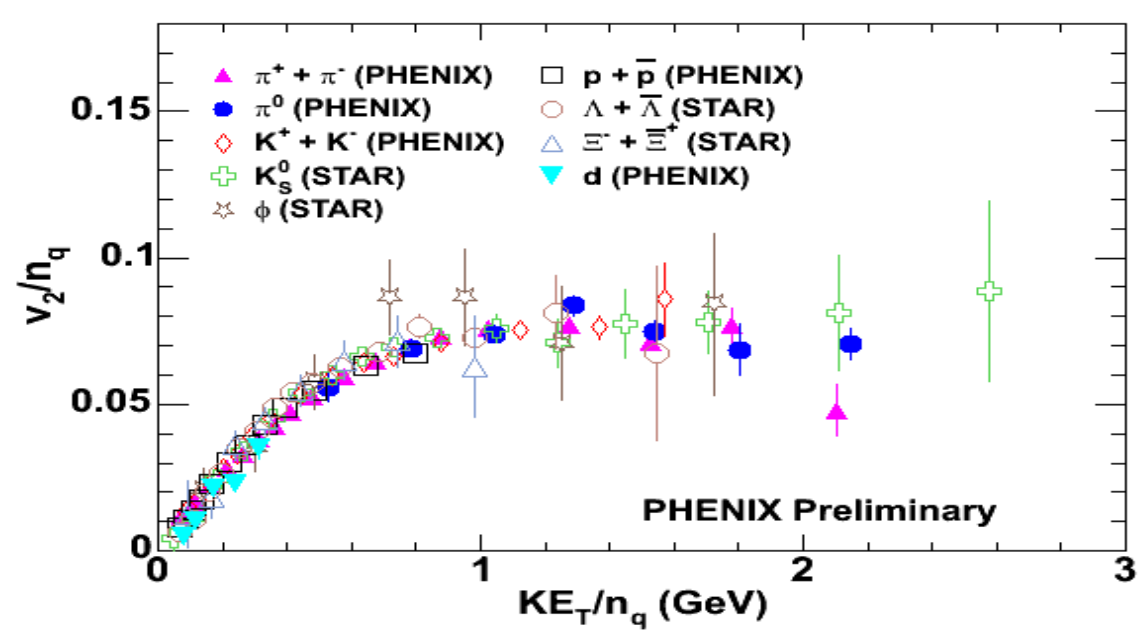

Fig. 4: The elliptic flow parameter plotted as $\mathrm{v}_{2} /$ quark against transverse energy/quark, to show the scaling with the number of valence quarks.

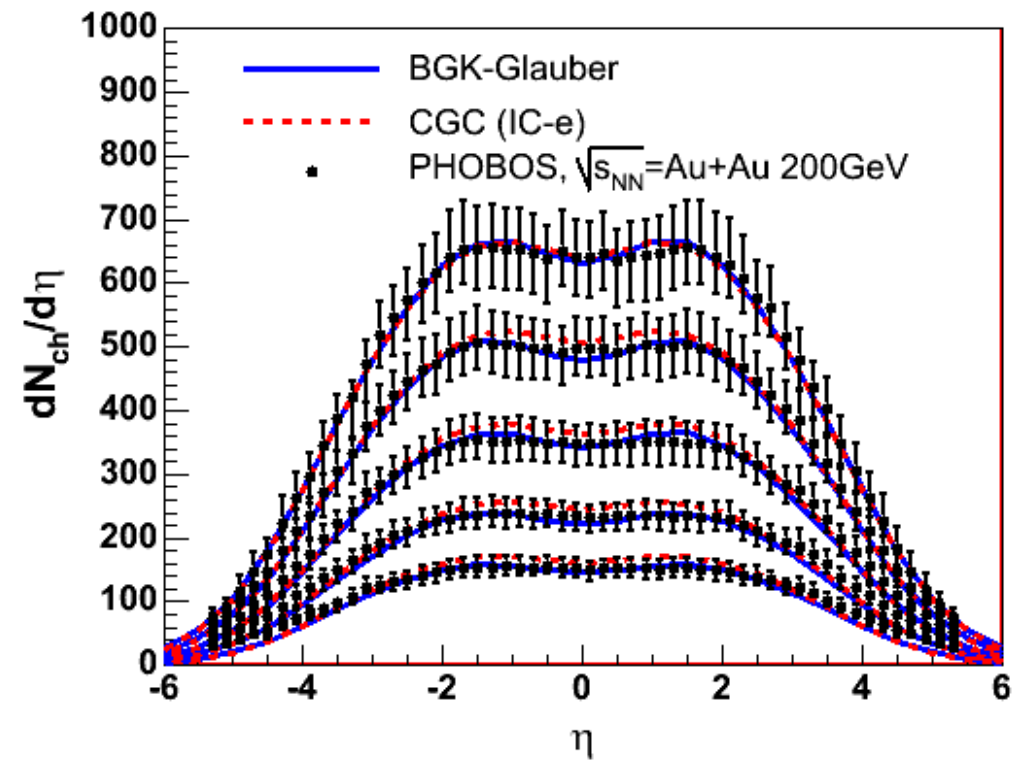

Fig. 5: $\mathrm{dN}_{\mathrm{ch}} / \mathrm{d} \eta$ as function of rapidity, $\eta$, for variety of collision centralities from PHOBOS, together with a fit using the Color Glass Condensate model, in which the saturation of the density of gluonic matter in the initial state leads to lower than expected particle multiplicity for central $\mathrm{Au}+\mathrm{Au}$ collisions at RHIC energy. 


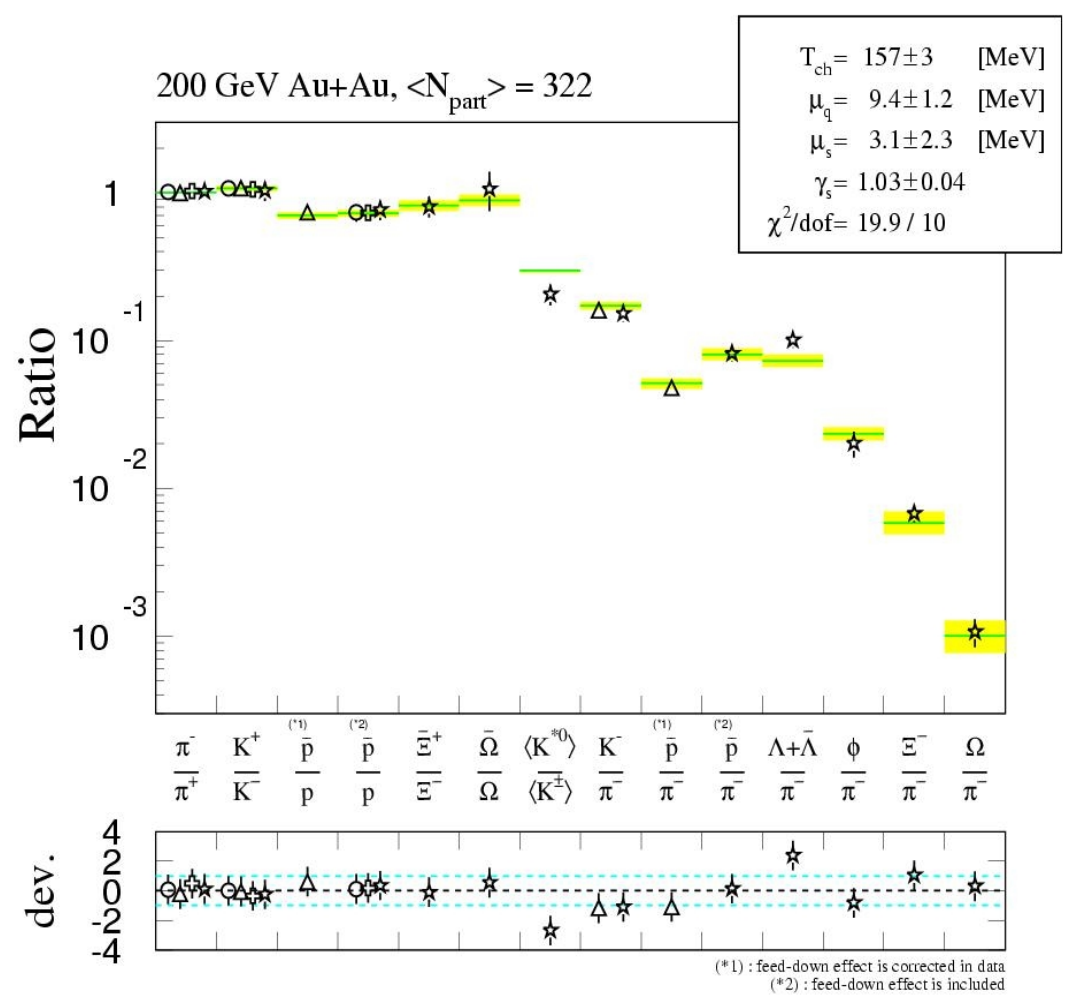

Fig. 6: Chemical equilibrium fit of hadron yield ratios (from all four RHIC experiments).

Let us now look at these results and their implications in some more detail.

Collective behavior: At midrapidity (and in not too peripheral collisions) the bulk of the produced hadrons with transverse momenta below about $2 \mathrm{GeV} / \mathrm{c}$, their abundances and the shapes of their transverse momentum spectra can be very well described by relativistic hydrodynamics for ideal fluids. While strong collective flow had been observed previously in lower energy heavy-ion collisions, hydrodynamic models were never before able to provide an equally successful quantitative description of the data. The best overall description of the RHIC data is obtained if ideal fluid dynamical evolution of a quark-gluon plasma during the early expansion stage is combined with a realistic hadronic cascade after hadronization, and if an equation of state like that obtained from Lattice QCD is employed. To reproduce the magnitude of the observed radial and elliptic flow it is necessary to assume that the produced matter thermalizes very quickly, on a timescale of less than $1 \mathrm{fm} / \mathrm{c}$, building up thermodynamic pressure whose gradients drive the collective expansion. Hydrodynamic calculations that reproduce the experimental data indicate that at thermalization time the energy densities must be at least 15 times that needed for color deconfinement and fireball temperatures are 2 times the critical temperature predicted by lattice QCD. In fact, even if one applies only the principle of energy conservation to the measured produced transverse energy in the collision, neglecting any energy lost to longitudinal work during the expansion, and uses any reasonable estimate for the initial volume of the fireball at thermalization, one also obtains a lower limit for the initial energy density which is about an order of magnitude above the critical value for deconfinement. 
The need for fast thermalization, the observation of large elliptic flow even for multi-strange (anti-) baryons and charmed hadrons, and the absence of any visible indication for a non-zero viscosity of the fluid during its early partonic (pre-hadronic) stage indicate that the extremely hot and dense medium created in the collision is a strongly coupled plasma. Its apparently almost perfect fluidity contrasts strongly with intuitive pre-RHIC expectations by most scientists in the field that the quark-gluon plasma would exhibit perturbative, gas-like behavior similar to electromagnetic plasmas known from everyday experience. The clarification of the strong-coupling mechanisms at work, the quantitative determination of the liquid's viscosity and a theoretical understanding of why it appears to be so small are key tasks for the RHIC II program.

Jet Quenching: The medium created in RHIC fireballs interacts strongly not only with its constituents, but also with hard colored internal probes, such as fast quarks and gluons created at the very beginning of the collision and propagating outward through the reaction zone. Hadrons with high transverse momenta which arise from the fragmentation of such hard partons are found to be suppressed in central $\mathrm{Au}+\mathrm{Au}$ collisions by a factor of $4-5$ relative to the experimental $\mathrm{p}+\mathrm{p}$ baseline, in contrast to direct photons which escape from the collision without further interaction and whose production rates and spectra agree well with expectations based on perturbative QCD. When triggering on a high- $\mathrm{p}_{\mathrm{T}}$ hadron with transverse momentum of up to $10 \mathrm{GeV} / \mathrm{c}$, its partner jet going off in the opposite direction is strongly quenched in central $\mathrm{Au}+\mathrm{Au}$ collisions: the yield of high- $\mathrm{p}_{\mathrm{T}}$ particles correlated with the trigger particle is reduced by a factor of 4 or more, while the energy is carried away by enhanced production of soft hadrons in the direction opposite to the fast trigger hadron. The average momentum of these soft hadrons approaches that of the thermalized medium as the collisions become more central and the fireball size increases. In addition, certain detailed structures in their angular distribution point to a possible collective or hydrodynamic response of the dense medium to the energy and momentum deposited by the quenched jet.

While not all of these features are quantitatively understood, theoretical estimates of the initial energy and particle density of the medium which are necessary to explain the observed high- $\mathrm{p}_{\mathrm{T}}$ hadron suppression agree with those required by the successful hydrodynamic description of the bulk of the matter. The important observation of an angular dependence of jet quenching relative to the reaction plane has opened the possibility to use this process as a tomographic probe for the properties of the dense medium created at RHIC. A second key component of the RHIC II program will be detailed studies of high- $\mathrm{p}_{\mathrm{T}}$ hadron production, including the detection of heavy mesons and multiple identified hadrons associated with jets, which enable us to turn jet emission tomography into a precision tool.

Hadron abundances and valence quark scaling: Whereas the kinetic freeze-out temperature (where elastic scattering stops) and the collective flow extracted from the final hadron spectra depend on collision centrality (more central collisions freeze out later, at lower temperature and with larger radial flow than peripheral collisions, consistent with theoretical ideas that describe kinetic freeze-out as a competition between local scattering and global expansion rates), the chemical decoupling temperature (where abundance changing interactions stop) extracted from the hadron abundances is found to be independent of collision centrality and thus insensitive to the expansion rate. This, together with its $160-170 \mathrm{MeV}$ value, strongly suggests that chemical freezeout is not controlled by inelastic hadronic rescattering processes, but by a phase transition in which 
the hadrons are born by a statistical (maximum entropy) process directly into a state which is relatively dilute and expands so rapidly that abundance-changing hadronic interactions cease immediately. In this way the final hadron abundances measure directly the critical temperature of the quark-hadron phase transition.

Further, evidence for an active role of deconfined, thermalized and collectively flowing quarks in hadron production comes from the observed valence quark number scaling of hadron yields and elliptic flow at intermediate $\mathrm{p}_{\mathrm{T}}$. While the ideal fluid description gradually breaks down above $\mathrm{p}_{\mathrm{T}}=$ $1.5-2 \mathrm{GeV} / \mathrm{c}$, the broadening of the baryon spectra by the strong radial flow remains visible at even larger transverse momenta, and this explains at least qualitatively the observed excess of (anti-)baryons over mesons in the intermediate $\mathrm{p}_{\mathrm{T}}$ region. A phenomenologically quite successful description is provided by the quark-coalescence model which posits that baryon production in this $\mathrm{p}_{\mathrm{T}}$ region proceeds via the coalescence of three quarks while meson production proceeds via the coalescence of a quark and an antiquark, each carrying a corresponding fraction of the final hadron's momentum. This process imprints the hydrodynamic flow characteristics of lowmomentum quarks onto the intermediate-momentum hadrons. As a result, hydrodynamic bulk particle production at low $\mathrm{p}_{\mathrm{T}}$ is separated from perturbative hard particle production at high $\mathrm{p}_{\mathrm{T}}$ by a novel and unexpectedly interesting intermediate $\mathrm{p}_{\mathrm{T}}$ region where quark coalescence and jet fragmentation compete with each other. This leads to interesting new phenomena whose detailed exploration and clarification will be a third major thrust of the RHIC II physics program.

Evidence for gluon saturation: At very high energies one is able to study the very wee parton (small-x) component of the hadron wave function. Theoretical investigations and experimental results from HERA have shown that the density of small-x gluons becomes very large. This led to the QCD based conjecture that these gluons, when measured at some fixed size scale, form a high energy density, saturated, highly coherent universal type of matter. There is a QCD based description of this matter, called the Color Glass Condensate. The earliest RHIC results on the dependence of particle multiplicity on centrality and energy were understood as arising from this high energy density state of saturated gluonic matter. Such matter can be probed in dA collisions by concentrating on kinematic regions sensitive to the small $\mathrm{x}$ gluon nuclear wave function. RHIC measurements showed a distribution of high transverse momentum particles whose dependence on rapidity, transverse momentum and centrality are consistent with the Color Glass Condensate hypothesis.

These observations are the first strong indication that gluon saturation effects play an important role in our understanding of the very early stages of heavy ion collision dynamics. Further exploration of the nature and manifestations of this novel type of physics in asymmetric collisions at forward rapidity will be a fourth key component of the RHIC II (and eventually eRHIC) physics program. 


\section{Exploring Heavy Ion Collisions... the Emerging Picture}

The time sequence of a central (head-on) gold-gold collision at RHIC

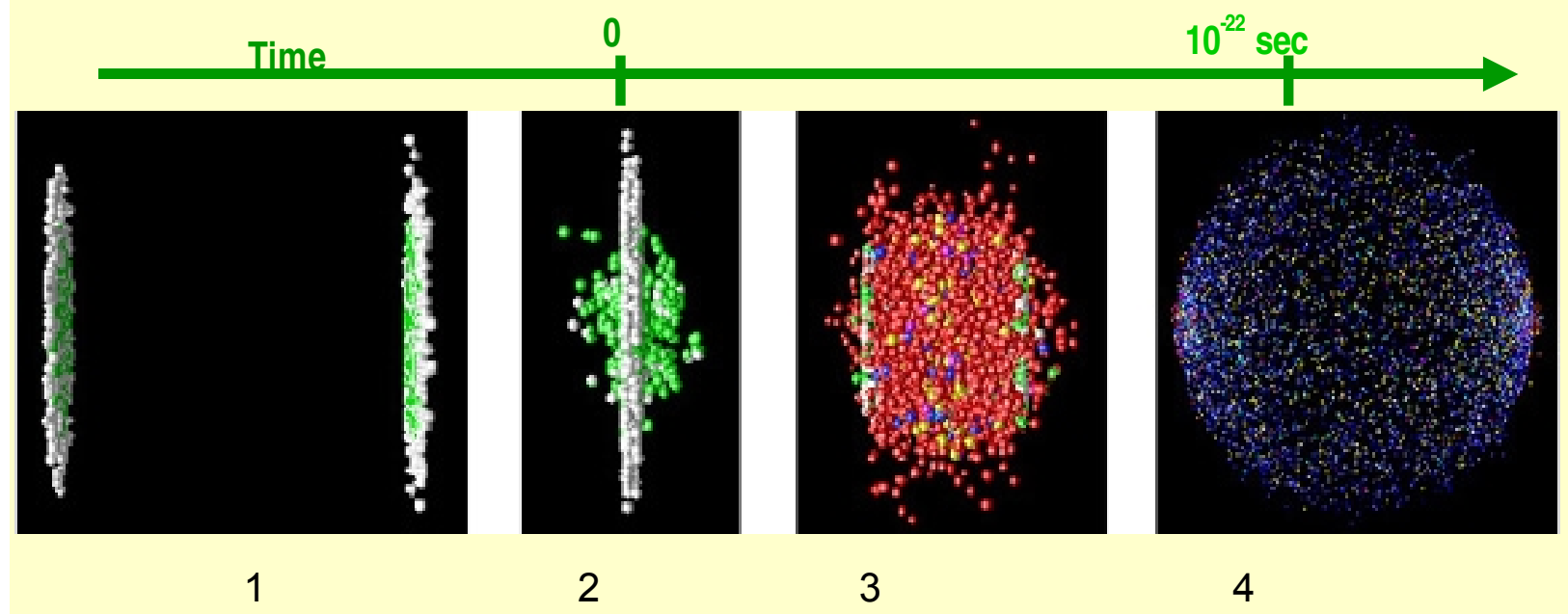

1.Initial state: The wave functions of the Lorentz-contracted incoming nuclei contain a high density of "soft" gluons. Data from high energy electron-proton collisions indicate that the density of interacting gluons grows with collision energy. In gold-gold collisions at RHIC the initial gluon density may reach a saturated value - a state described by theorists as a Color Glass Condensate. The interaction of these high-density "walls" of gluons describes the initial state of the collision.

2. Impact: The two nuclei collide, heating the local QCD vacuum through the interaction of intense color fields to produce a dense, pre-equilibrium state of quarks, anti-quarks and gluons. Sometimes high energy quarks and gluons are formed, that will produce "jets" of hadrons as they propagate through the dense matter. Pairs of heavy quarks, charm and beauty, are formed in this initial stage of the collision.

3. The plasma phase: The hot, dense matter, consisting of quarks, anti-quarks and gluons, reaches thermal equilibrium almost instantaneously. It expands and cools through very strong collective interactions behaving like an ideal hydrodynamic fluid - a "perfect liquid" with nearly zero viscosity. High-energy quarks and gluons interact with this medium, providing a probe of its density and bulk properties through the measurement of jet phenomena. Heavy quarks and antiquarks combine with the more abundant light quarks in the medium, or with each other, to form bound states whose spectrum reflects the color screening effects of the medium (analogous to Debye screening in atomic media).

4. Freeze-out: As the expanding matter cools below the QCD critical temperature, quarks and antiquarks coalesce to form hadrons, and mutual interaction quickly ceases. This hadronic final state radiates the particles seen in experimental detectors. The properties of this final state anisotropies in the spatial distribution of hadrons, and the relative abundance of particles in terms of their quark constituents -- retain a memory of the conditions at the time of freeze-out: a consequence that is analogous to the imprint of the early universe on the observed cosmic microwave background. 


\section{How Do We Know It Flows?}

Elliptic flow has proven to be very important at RHIC because it is related to the pressure of the hot, dense matter at the earliest times. When two heavy nuclei collide, they can strike each other head on in a "central" collision, they can barely graze each other in a peripheral collision, or anywhere in between. For the in-between cases, the reaction zone has an ellipsoidal "almond" shape, as seen in the figure at the right. This leads to asymmetric pressure gradients that are more effective at pushing particles out along the "reaction plane" direction (the $x$-direction in the

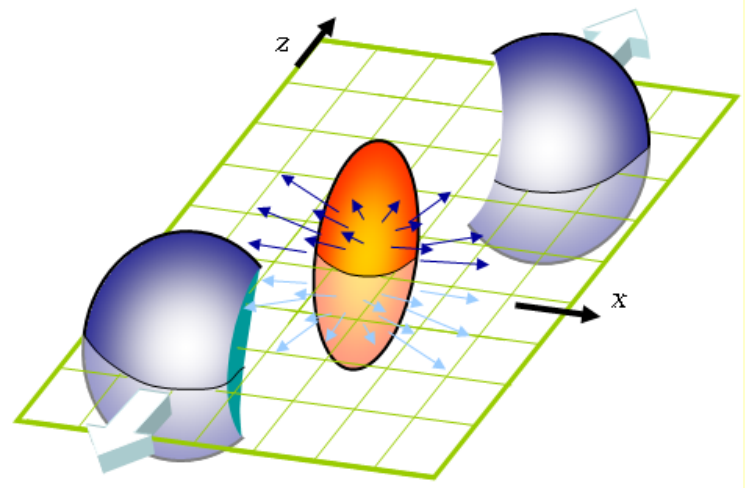
figure) rather than perpendicular to it. For collisions of identical nuclei such as $\mathrm{Au}+\mathrm{Au}$, the mid-rapidity $\left(\theta \approx 90^{\circ}\right)$ particle density at any given angle $\varphi$ can be described by:

$$
\frac{d N}{d \varphi} \propto 1+2 v_{2} \cos 2\left(\varphi-\Psi_{\text {plane }}\right)+\ldots
$$

where $\Psi_{\text {plane }}$ specifies the reaction plane angle and $v_{2}$ measures the pressure-driven asymmetry, i.e. the "elliptic flow".

At RHIC, $v_{2}$ is so large that up to $30 \%$ more particles are emitted in the reaction plane than out of it. This makes it straightforward to measure elliptic flow by dividing the produced particles for

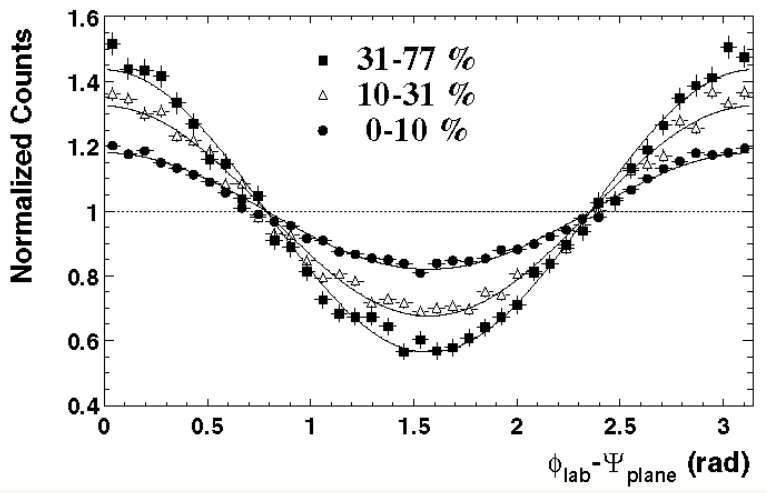
each event into two groups. The first group is used to determine the reaction plane orientation. The elliptic flow of the particles in the second group can then be measured. A typical case is illustrated in the figure on the left, where the elliptic flow of charged particles with intermediate transverse momenta $\left(2<p_{T}<6\right.$ $\mathrm{GeV} / \mathrm{c}$ ) has been measured relative to the reaction plane determined by the low transverse momentum $\left(p_{T}<2 \mathrm{GeV} / \mathrm{c}\right)$ particles. Data are shown for three centrality classes with, for example, $0-10 \%$ being the $10 \%$ of the collisions

that are most central. The cos $2 \varphi$ dependence is clearly seen. Furthermore, the asymmetry is larger for the more peripheral collisions $(31-77 \%)$, where the reaction zone eccentricity is larger, than it is for the head-on collisions $(0-10 \%)$, where the reaction zone is nearly spherical.

Why do we care? As the dense matter evolves, the asymmetric pressure gradients push it toward spherical symmetry. Thus, elliptic flow provides a window to study the equation of state of the system at early times. Hydrodynamic calculations have been found to provide a good description of the observed elliptic flow, but only by assuming the system thermalizes extremely rapidly and then evolves as a nearly perfect liquid. 


\subsection{Spin physics:}

In parallel with the above discoveries in heavy-ion collisions, RHIC has commissioned the world's first polarized proton collider. This technological achievement permits, for the first time, study of spin observables in polarized hadron-hadron collisions in the regime where perturbative QCD (pQCD) provides a reliable framework for interpretation. Such collisions provide critical complements to electromagnetic probes of nucleon spin structure, such as deep inelastic lepton scattering (DIS). The latter studies, when carried out with polarized lepton beams and polarized nucleon targets, have firmly established that, in a partonic description, only a small fraction $(\sim 20-$ $30 \%$ ) of the proton's spin can be attributed to a preferential alignment of the spins of all quarks and antiquarks in the proton. The remainder must be sought in either the preferential alignment of gluon spins or the orbital motion of quarks or gluons within the nucleon. The RHIC spin program addresses the first of these possible origins directly by probing gluon spin preferences at leading order with color forces, and illuminates the second in unique ways.

During the development of polarized collider capabilities, RHIC $p+p$ measurements have already established a few important milestones on the path toward the above goals. They have demonstrated that the cross sections for several of the reaction channels intended to provide gluon sensitivity are, indeed, well reproduced by next-to-leading-order (NLO) pQCD calculations over the entire transverse momentum range of interest. The agreement is illustrated in Fig. 7 by PHENIX results for inclusive production of $\pi^{0}$ and direct photons at $\sqrt{s}_{\mathrm{s}}=200 \mathrm{GeV}$. This success sets the stage for the extraction of new structure information by comparison of theory and experiment for polarization observables. RHIC measurements have furthermore demonstrated that sizable spin effects can be observed in hadronic collision regimes where pQCD is applicable. This is illustrated in Fig. 8 by the single-spin transverse asymmetries measured for forward $\pi^{0}$ production in STAR. These transverse asymmetries can arise from both transverse spin orientation preferences and transverse motion preferences of partons within a transversely polarized proton, with the latter preferences related to parton orbital angular momentum. Follow-up measurements planned for the next few years at RHIC will distinguish between these competing origins of transverse spin asymmetries.

Initial measurements of two-spin longitudinal asymmetries sensitive to the helicity preferences of gluons have begun at RHIC. With the statistical precision achieved in the 2005 run, these asymmetries (see Fig. 9) already appear to rule out extreme scenarios in which gluon polarization would account, even at low momentum transfer scales, for greater than $100 \%$ of the proton spin, only to be partially compensated by orbital contributions. Such scenarios had been considered in theoretical speculations concerning shielding effects that might have explained the small contributions from quark and antiquark spins.

A quantitative determination of the gluon polarization as a function of gluon momentum fraction, and eventually of the integral gluon contribution to proton spin, is anticipated from RHIC spin measurements that will span the RHIC-I era, including runs at both $\sqrt{s}_{\mathrm{s}}=200$ and $500 \mathrm{GeV}$ and including reaction channels both abundant (inclusive $\pi^{0}$ and jet production) and rare (e.g., direct photon production in coincidence with jets). A key component of the future spin physics program, which will begin as soon as the necessary detector upgrades are in place, will be measurements of 
single-spin asymmetries for $\mathrm{W}$ boson production to determine the contributions that $\bar{u}$ and $\bar{d}$ antiquarks make to the proton spin. The RHIC II luminosity upgrade will then enable these measurements to be extended to include the separate contributions made by strange quarks and antiquarks.
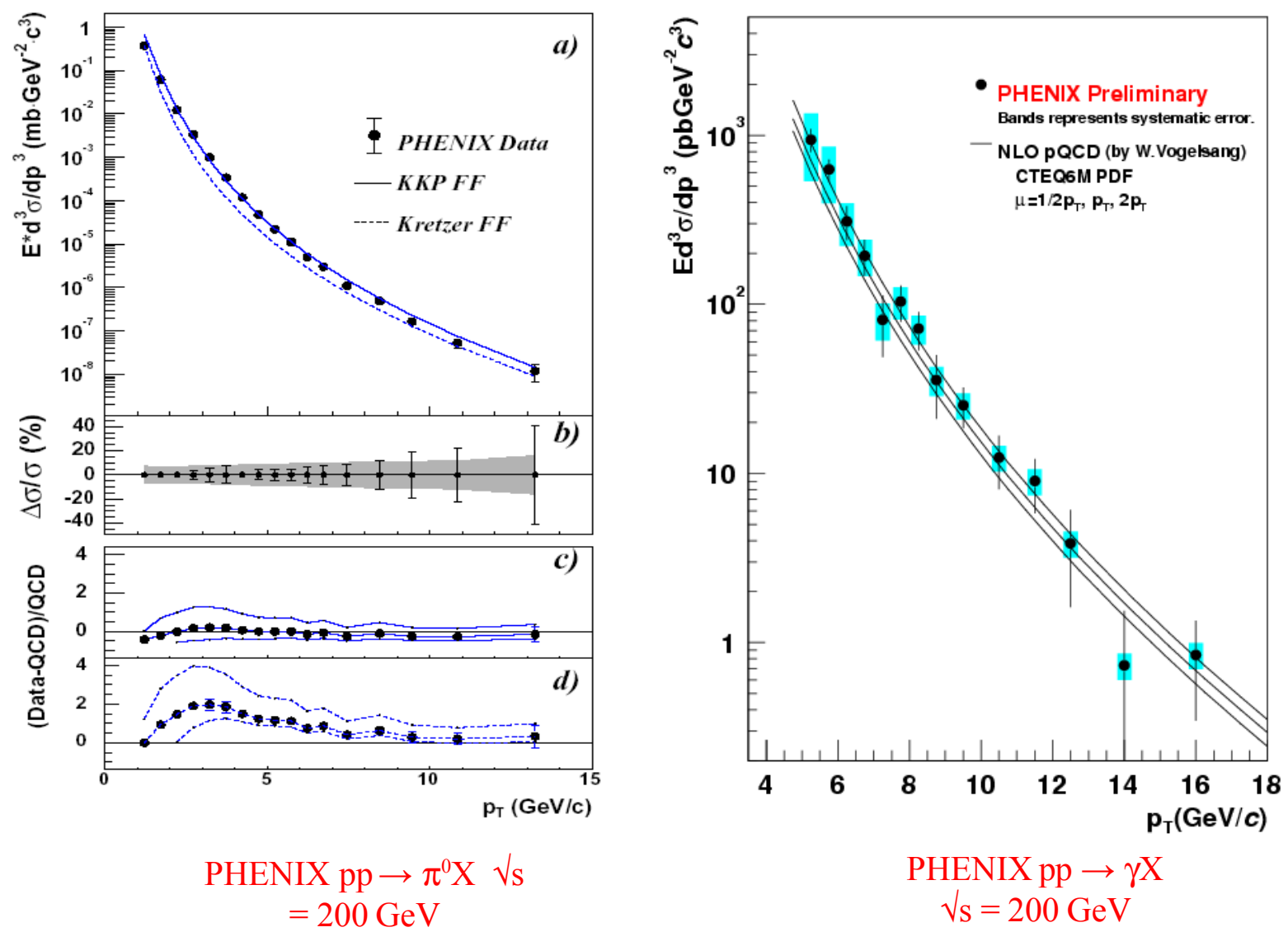

Figure 7. Comparison of differential cross sections measured by PHENIX for inclusive production of $\pi^{0}$ (left) and direct $\gamma$ (right) at $\sqrt{s}_{\mathrm{s}}=200 \mathrm{GeV}$ with next-to-leading-order pQCD calculations. The different curves utilize different parton fragmentation functions (left) or different factorization scales (right). 

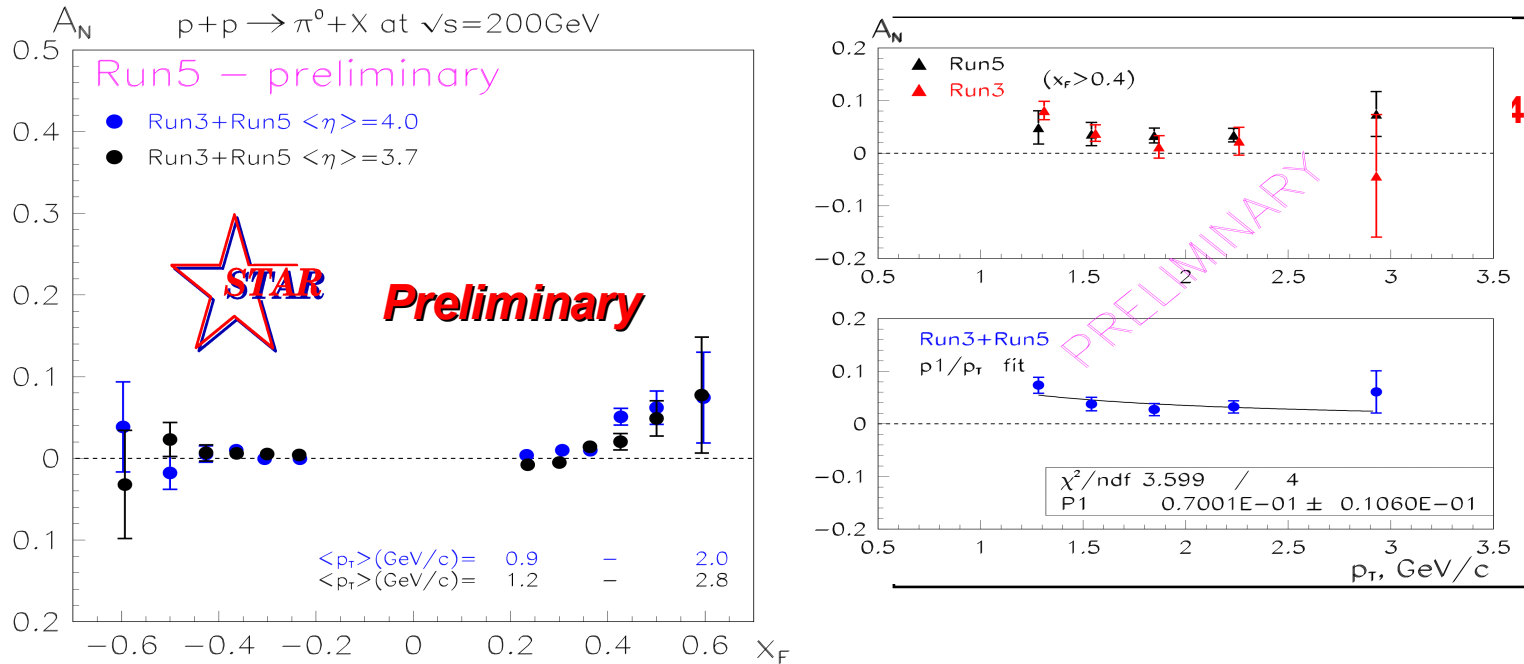

Figure 8. Transverse single-spin asymmetries for inclusive forward $\pi^{0}$ production measured at STAR, showing their dependence on $\mathrm{x}_{\text {Feynman }}$ (left) and $\mathrm{p}_{\mathrm{T}}$ (right). The value of $\mathrm{x}_{\mathrm{F}}$ is (approximately) the difference in momentum fraction of the interacting partons. The sizable asymmetries seen at positive $\mathrm{x}_{\mathrm{F}}$ are reminiscent of values measured at bombarding energies an order of magnitude lower, but at RHIC are amenable to pQCD interpretation.

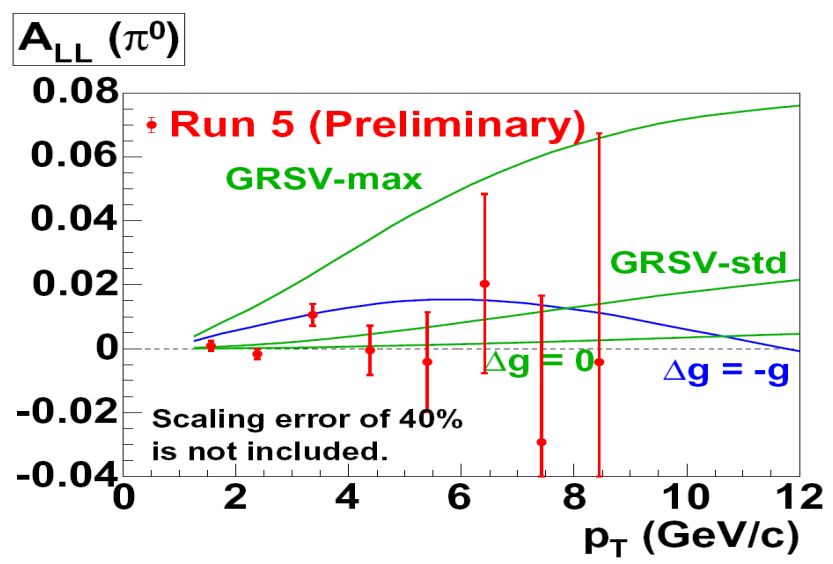

Figure 9. Preliminary PHENIX results for the two-spin longitudinal asymmetry for mid-rapidity inclusive $\pi^{0}$ production, compared to next-to-leading-order pQCD calculations assuming various scenarios for gluon polarization. Neither the blue curve nor the upper green curve, assuming, respectively, $-100 \%$ and $+100 \%$ gluon polarization at a low momentum transfer scale, are consistent with the results. At the presently available statistical precision the results do not yet discriminate among less extreme gluon polarization scenarios. 


\section{QCD and the Spin of the Proton}

Quantum chromodynamics describes the proton as containing two valence up quarks and one valence down quark, bound together by gluons. In the naïve quark model, properties of the proton like its charge and magnetic moment arise from these three valence quarks. Likewise, for many years it was assumed that the spin-1/2 of the proton arises from the sum of the spins of the three spin-1/2 quarks. Therefore, it was a great surprise in 1988 when the European Muon Collaboration announced measurements of scattering of polarized muons on polarized protons that implied only $20-30 \%$ of the proton's spin is carried by quarks and antiquarks. This result has come to be known as the "spin crisis".

So where does the spin of the proton come from? In QCD, the choices are limited. The quarks and antiquarks carry spin $1 / 2$; the gluons carry spin 1 ; the quarks and gluons can also carry orbital angular momentum contributions. The proton spin must be a combination of these.

How can we learn about the components of the proton spin? At RHIC, hard scattering cross sections in $\mathrm{p}+\mathrm{p}$ collisions have been shown to agree with predictions from perturbative QCD. In perturbative QCD, these cross sections include large contributions from gluon+gluon and quark + gluon scattering processes. It is possible to choose specific processes that are sensitive to the relative spin directions of the interacting quarks or gluons, and to measure the yields of these processes for different orientations of the spins of the colliding polarized protons. Any observed "asymmetries" in these yields are due to net polarization of the quarks, gluons or orbital angular momenta within the polarized proton.

To date, experiments have provided only very loose constraints on the gluon contribution to the proton spin. This possibility is the primary focus of the first phase of the RHIC spin physics program. Initial results make it unlikely that the gluons contribute "too much" spin to the proton, as some models had predicted. But we don't yet know whether they contribute enough to resolve the spin crisis or not.

To explore the possibility that orbital angular momentum plays a significant role in generating the proton spin, additional measurements are underway to see if the quarks and gluons in a spin-up proton have a preferential tendency to move toward beam-left vs. beam-right.

In the future, the RHIC spin program will explore the contributions that $\bar{u}$ and $\bar{d}$ antiquarks from the "Dirac sea" make to the proton spin, and the RHIC II luminosity upgrade will enable measurements that are sensitive to the contribution from strange "sea" quarks. 


\section{The RHIC facility: evolution and future}

Since the first collisions at RHIC in June 2000, the facility has compiled a series of groundbreaking measurements that have changed dramatically the scientific complexion of high energy nuclear physics. Designed to explore new extremes of collision energy, RHIC has provided the first-ever colliding beams of heavy nuclei and spin-polarized protons at high energies. These first runs were characterized by a wide range of operating modes, a rapid development of machine capability - exceeding design performance for heavy ions after the first three runs -- and a rich harvest of physics discoveries that have opened up fundamentally new avenues of research in QCD.

A key element of the successful discovery phase of the RHIC program has been the ability of the collider to switch rapidly between species and energies, providing nucleus-nucleus, nucleon (deuteron)-nucleus, and polarized proton-proton collisions efficiently and "on-demand", as called for by emerging physics results. Another has been the rapid processing, analysis, and publication of physics results from very large data samples, with detectors designed to provide sensitivity to a wide spectrum of predicted phenomena in an exploratory program of broad scope, all in a largely unknown landscape.

The initial suite of RHIC detectors consisted of two large, general purpose spectrometers of quite different design but with similar, far-reaching ability to probe the detailed characteristics of highenergy collisions with extremely high particle densities (PHENIX and STAR), and two smaller, more specialized detectors (BRAHMS and PHOBOS) designed to quickly map out specific global features of these collisions. PHENIX and STAR were among the first collider detectors to fully exploit custom integrated circuit technology for readout electronics, allowing fine-grained, high channel-count detector systems capable of fully resolving events with thousands of charged particles in the final state.

Such detectors, operating at high collision rates, provide an enormous challenge for recording, archiving, and analyzing data in great volume. The RHIC Computing Facility (RCF) has provided dedicated computing resources, tailored to the specific recording and analysis needs of each experiment, to ensure efficient and rapid processing of data volumes that have reached hundreds of Terabytes for each annual RHIC run.

The ability to quickly analyze data has been a cornerstone of the RHIC program to date. New experimental results have appeared within a few weeks or months after each data run, with important new discoveries confirmed by multiple measurements among the experiments. This has quickly galvanized the theory community to move forward with reliable guidance from experiments, and has allowed for rapid scientific feedback into the planning for RHIC operations. A notable case in point is the quick decision to collide deuterons on gold after the discovery of jet quenching in the 2002 run, thereby resolving almost immediately the question of whether this effect was the result of energy loss in a final-state medium or shadowing in the initial state.

Measurements at RHIC have now reached the stage at which rare processes, the hallmark of highenergy collider particle physics, are precisely the probes that will allow definitive measurements of the quark gluon plasma and other new phenomena in the physics of high energy density matter. 
From the outset the analysis of RHIC data has exploited hard-scattering phenomena to begin examining the properties of the hot dense matter produced in nuclear collisions, and the most recent high-statistics data sets have begun to yield information on the bulk properties of this matter through the observation of the heavy quarks, charm and beauty. This gives a clear indicator for the future evolution of RHIC.

The two small experiments, BRAHMS and PHOBOS, have achieved their goals for data collection and completed their runs. Further RHIC runs are focused on the two large experiments, STAR and PHENIX, which are now engaged in a series of significant upgrades planned for implementation over the next 5-6 years. These upgrades are aimed specifically at providing enhanced sensitivity to the rare probes described in this report, and many of them are crucial to utilizing significant improvements in machine luminosity.

Many of the fundamentally important measurements described in this report call for data samples requiring integrated luminosities corresponding to $\sim 10 \mathrm{nb}^{-1}$ in $\mathrm{Au}-\mathrm{Au}$ collisions (equivalent to $\sim 400$ $\mathrm{pb}^{-1}$ in nucleon-nucleon collisions). Such data samples, along with the necessary comparison data, would take several decades to accumulate at present luminosities. The plan for RHIC II is to make these measurements achievable by increasing the RHIC heavy ion luminosity by an order of magnitude through electron cooling of the ion beams.

An important scientific issue for which the flexibility of the facility is crucial is the effort among theorists, experimenters, and RHIC machine physicists to mount a search for the QCD critical point using the collider and the STAR and PHENIX detectors to scan collision energies below the RHIC injection energy. Theory and experiment both indicate that the matter produced in topenergy RHIC collisions, at near-zero baryo-chemical potential ( $\mu_{\mathrm{B}}$ less than $\left.\sim 50 \mathrm{MeV}\right)$ undergoes a rapid cross-over from quark gluon plasma to a hadron gas, but does not feature a first-order phase transition. The consensus at the workshop held at BNL in March, 2006 was that the critical point, marking the onset of a first-order phase transition, is accessible to heavy ion collisions if it lies in the range $\mu_{\mathrm{B}}<500 \mathrm{MeV}$, a range that could in principle be covered in collisions at RHIC. Studies are presently underway to extend the energy range of RHIC collisions downward and improve the low energy luminosity to allow such a scan with the existing detectors. The collider environment provides a significant advantage in this case because, unlike fixed-target measurements, most systematic effects are independent of beam energy. Direct measurements revealing the existence and properties of the critical point would provide an essential quantitative understanding the QCD phase diagram. (See "Can we discover the QCD critical point at RHIC?" https://www.bnl.gov/riken/QCDRhic/ )

Plans to provide higher luminosity and increased flexibility for the colliding beams are as follows:

\section{Enhanced luminosity in the near term}

Upgrades of the RHIC vacuum system are currently being undertaken that will enable increases in both the Au-Au and p-p luminosity. The target values, expected to be achieved by 2008, are as follows (luminosity averaged over one store):
$\mathrm{Au}-\mathrm{Au}$ at ${\sqrt{\mathrm{S}_{\mathrm{NN}}}}=200 \mathrm{GeV} / \mathrm{n}-\mathrm{n}$ pair
p-p at $\sqrt{s}_{\mathrm{s}}=200 \mathrm{GeV}$
$8 \times 10^{26} \mathrm{~cm}^{-1} \mathrm{sec}^{-1}$
$\mathrm{p}-\mathrm{p}$ at $\sqrt{\mathrm{s}}=500 \mathrm{GeV}$
$6 \times 10^{31} \mathrm{~cm}^{-1} \mathrm{sec}^{-1}$
(4x original design)
(16x original design)
$1.5 \times 10^{32} \mathrm{~cm}^{-1} \mathrm{sec}^{-1}$
(16x original design) 
For proton-proton collisions, the polarization is expected to reach $70 \%$.

\section{A new ion injector, EBIS}

A new Electron-Beam Ion Source, EBIS, will replace the 35-year old Tandem electrostatic accelerators, resulting in simpler, more reliable operation at reduced cost. EBIS is expected to begin construction in 2007, and should be commissioned in 2009. Among other improvements, EBIS will offer high-intensity beams of Uranium, as well as polarized ${ }^{3} \mathrm{He}$ ions.

\section{Development and implementation of electron cooling for RHIC II}

The enabling technology for RHIC II is electron cooling of the full energy beams, to mitigate the beam blow-up effects of Coulomb forces among the tightly bunched, highly charged ions. This will allow a ten-fold increase of the average heavy ion luminosity over the "enhanced" value for $\mathrm{Au}-\mathrm{Au}$ given above. For polarized protons a luminosity increase of a factor 2-3 is expected, using cooling to increase the brightness (protons/unit area) of the beams at injection. Electron cooling at RHIC is based on a high-intensity, low emittance superconducting electron gun, and an Energy-Recovery Linac (ERL), also superconducting. Both critical components are under development at BNL. Brookhaven expects to demonstrate feasibility of the RHIC II ecooling upgrade in 2006, after which construction could start in 2009, with commissioning in 2012.

With the implementation of electron-cooled ion beams, RHIC will be in a position to complete its evolution as a QCD Laboratory with the addition of an electron beam to provide high-luminosity electron-ion and polarized electron-proton collisions at high energy. Development studies are under way for this final step, the eRHIC project, which will include a dedicated intersection region and new detector for electron collisions with the RHIC beams. 


\section{Fundamental questions for the next ten years at RHIC}

The $20^{\text {th }}$ century witnessed spectacular progress in understanding the elementary constituents of matter and the forces between them. That work has culminated in the Standard Model, which describes these forces as interactions between fermions mediated by the exchange of gauge bosons. In contrast to the special case of electromagnetism, the electroweak and the strong interactions are formulated as non-Abelian gauge theories which require interactions also among the gauge fields, which in turn give rise to non-linear phenomena of fundamental importance. Among these are the matter-antimatter asymmetry of the universe (electroweak) and the origin of $98 \%$ of the mass of ordinary matter (strong). The theory of the strong interaction, Quantum Chromodynamics (QCD) is particularly challenging, since the coupling between the quanta is very large in many interesting regimes, and thus defies analysis with perturbative tools. Nonetheless, QCD has proven accessible to detailed experimental investigation at RHIC and to quantitative numerical studies using Lattice Gauge Theory. The physics opportunities to be pursued may be summarized in terms of four fundamental questions:

\subsection{What are the phases of QCD matter?}

Soon after QCD was developed, it was conjectured that a phase transformation from distinct ordinary hadronic constituents to a state of deconfined quarks and gluons should be expected at high temperatures and/or densities. Subsequent theoretical investigations have refined this conjecture, leading to predictions of a rich phase structure as a function of temperature and baryon density (see Fig. 10). The initial experimental results from the RHIC program have demonstrated unequivocal evidence for a new state of partonic matter with novel and unexpected properties, and have identified fruitful avenues for further investigation in the fundamental structure of the dense QCD medium.

While it is clear that the matter formed at RHIC has unique properties, fundamental questions remain to be investigated with an upgraded facility. What is the structure of the matter, and what are the relevant degrees of freedom? Are there bound states even above the transition temperature? Is chiral symmetry restored? What is the equation of state of the matter? What are its transport properties? Of particular interest is the ratio of viscosity to entropy, which has been conjectured to obey a universal lower bound. The prospects to study such quantities in a fundamental theory have excited interest well beyond nuclear physics, with emerging connections from ultracold atomic systems in traps to strongly coupled dusty plasmas to computations via string theory analogs of strongly coupled gauge theories.

An upgraded RHIC facility allows detailed investigation into the high temperature phase structure of QCD. Systematic exploration is needed to search for the intriguing features, such as the critical point and the subsequent appearance of a first-order phase transition, which are essential but as yet unobserved aspects of our current understanding of QCD. The key control parameter here is the baryon density, which may be increased by lowering the collision energy and/or by measuring at forward rapidities. Even higher baryon densities may be achieved by applying these same techniques to colliding beams of uranium provided by the new ion source currently under construction. 


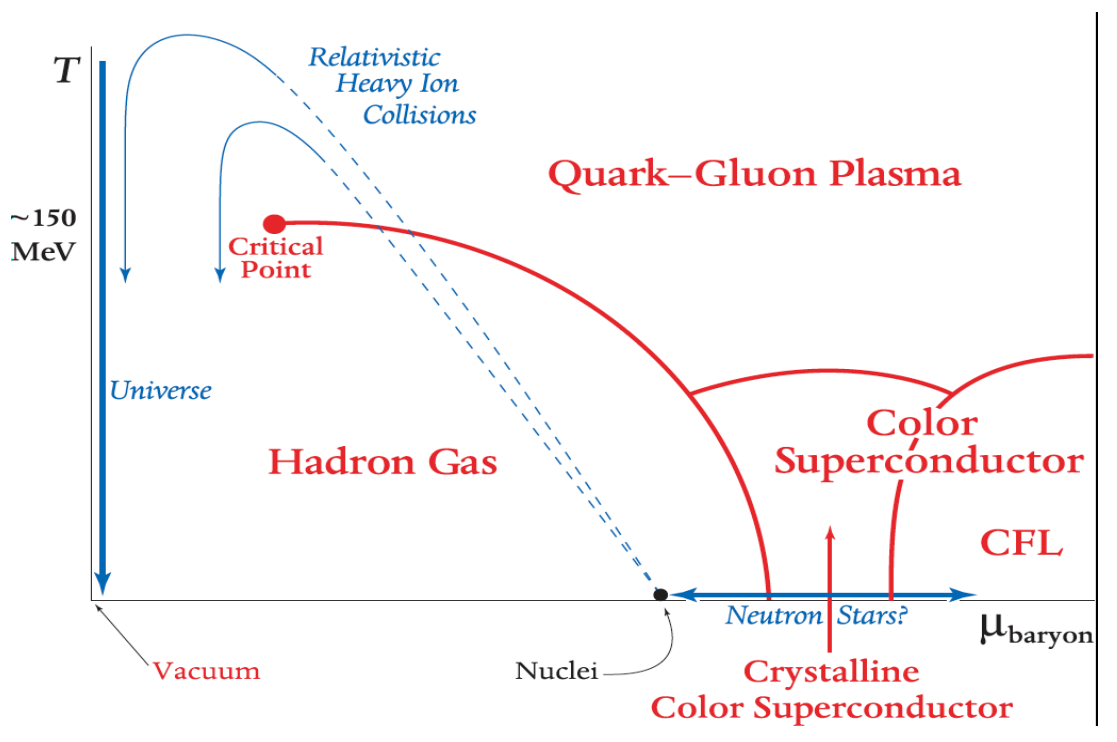

Fig. 10: The present picture of the QCD phase diagram of temperature versus baryonic chemical potential (a measure of the net baryon number). The line of first order deconfinement phase transitions terminates at the critical point where the phase transition is second order. The phase transition is a rapid crossover to the left of the critical point.

\subsection{What is the wave-function of a heavy nucleus?}

General arguments suggest that the growth of gluon number density at small momentum fraction $x$ in the proton cannot continue indefinitely, but must saturate. Heavy nuclei at high energy provide an ideal laboratory to study this phenomenon, since the overlap of gluons from the many nucleons that form the nucleus lower the scale for its onset. For example, one QCD-based theory, the Color Glass Condensate (CGC), postulates a universal wave function for gluonic matter in the saturation limit. Models based on saturation have scored successes in describing certain aspects of the RHIC data, such as the relatively low multiplicities of produced particles and the suppression of particle production in the forward direction. Verification of the CGC would establish the presence of classical gluon fields in cold nuclear matter; as such it would represent a fundamental advance in our understanding of QCD in extreme conditions and of the high energy limit of the strong interaction. Just as importantly, precise measurement of the gluon distribution in nuclei at low momentum fraction is essential for understanding the initial state for heavy ion collisions at both RHIC and LHC energies.

\subsection{What is the wave-function of the proton?}

The wave-function of the hydrogen atom was computed almost simultaneously with the introduction of the Schrodinger equation. However, 30 years after the formulation of the QCD Lagrangian, a corresponding quantitative computation of the proton's ground state remains elusive. Instead, our knowledge of the motion of quarks and gluons within the proton has been derived 
from precision measurements of deep-inelastic scattering (DIS) combined with analyses based on the evolution equations of QCD. Strikingly, this work has demonstrated that only $20-30 \%$ of the proton's spin results from the spins of the quarks within it. The search for the major contributions to the proton spin has now turned to the role of gluon spin, the polarization of the sea quarks, and orbital angular momentum. A polarized proton collider is uniquely suited for this task, because it enables us to probe the spin distributions of quarks, antiquarks, and gluons in a kinematic regime that permits sound theoretical interpretation based on perturbative QCD methods. RHIC, as the world's first and only polarized proton collider, provides an unparalleled opportunity to pursue these studies essential to our understanding of proton structure.

\subsection{What is the nature of non-equilibrium processes in a fundamental theory?}

Out-of-equilibrium processes have profound impact on the formation of structure and matter in the early universe. A spectacular example of the role of a non-thermal process is baryon number generation in the early universe, which depends in an essential way on deviations from thermal equilibrium. This phenomenon occurs at the electroweak scale, and as such is not accessible to direct experimental investigation. However, a rather precise analogy exists between baryon number generation via the electroweak phase transition, and parity and/or CP violation via the axial anomaly in the case of a second-order QCD phase transition. Unlike the electroweak phase transition, characterized by temperatures of about $100 \mathrm{GeV}$, the QCD transition temperature of "only" $170 \mathrm{MeV}$ has proven to be experimentally accessible, and in fact will be exceeded more than $10^{4}$ times per second in nuclear collisions at RHIC II luminosities.

The generation of thermal behavior, and more generally the production of entropy, from an initial quantum state remains an open and difficult question. The data from RHIC clearly establish that thermalization develops in the earliest stages of the collision, yet the precise mechanism is not understood. A variety of approaches is currently being investigated, ranging from description of the initial states as Color Glass Condensates to exponential degradation of the non-isotropic initial states via plasma instabilities to interpretations based on Unruh/Hawking radiation experienced by decelerating observers. 


\section{The future physics program at RHIC}

Each of the four fundamental questions outlined in the previous section can be associated with a set of future measurements at RHIC. In this section we briefly describe the measurements associated with each of the questions, and how they benefit from the planned detector upgrades and from the RHIC II luminosity upgrade. 


\subsection{Equation of state and the QCD phase diagram}

Here we describe future measurements addressing the first of the fundamental questions, introduced in section 4.1, "what are the phases of QCD matter?". This is a broad topic, and the following sections are organized as follows:

- Because it must be demonstrated that the system is thermalized before it makes sense to try to characterize the state of the matter, we begin with a discussion of some dynamical considerations - evidence of thermalization, thermalization timescale and mechanisms, diffusion and viscosity.

- Together, the temperature and energy density define the equation of state of the matter formed at RHIC. We discuss what can be obtained from the temperature and energy density, and how they might be determined experimentally.

- We discuss the importance of the QCD critical point in mapping out the phase diagram, and how it might be investigated by varying the baryon chemical potential using colliding beams of different energy and species.

- We describe programs to measure medium modifications of hadron properties in hadronic matter close to the transition temperature.

- We discuss the possibility that direct evidence of deconfinement can be seen in the disappearance of certain quarkonium states.

- We discuss the importance of understanding the process by which the partonic medium converts to hadrons.

\subsubsection{Dynamical considerations}

Establishing the existence of a thermalized system is an important prerequisite for a new state of matter to be formed. We examine here the evidence that a thermalized system has been formed in $\mathrm{A}+\mathrm{A}$ collisions at RHIC, the time scale for thermalization to occur, and the experimental observables that may establish links to theoretical models of thermalization. Note that the discussion in this section (5.1.1) has strong overlap with part of the discussion in section 5.4, where non-equilibrium processes are discussed.

\subsubsection{Evidence for thermalization}

Hadron abundances measured in relativistic heavy ion collisions have been successfully described using statistical thermal models (see Fig. 6). The inference from these studies is that the system is in chemical equilibrium with a chemical freeze-out temperature of $\mathrm{T}_{\text {chem }} \sim 170 \mathrm{MeV}$, independent of collision system and collision centrality. This temperature agrees with the value for the quarkhadron phase transition temperature predicted by Lattice QCD. The system expansion and hadronic re-scattering may still continue after chemical equilibration. The temperature at kinetic freeze-out, when hadrons stop interacting, is reflected in the slopes of the hadron momentum distributions and it can be extracted by comparing the measured spectral shapes of identified hadrons to a hydrodynamic model that has been tuned to reproduce the data. The model shows that it takes about $9-10 \mathrm{fm} / \mathrm{c}$ until the fireball has become sufficiently dilute to completely convert to hadronic matter, and another 7-8 fm/c to completely decouple at a temperature of $\mathrm{T}_{\text {kinetic }} \sim 100 \mathrm{MeV}$. The success of the statistical thermal models and the hydrodynamic description of single particle 
spectra and yields implies that the matter produced at RHIC has reached thermal equilibrium at some stage of its evolution.

In the future, another test of chemical equilibrium can be carried out at RHIC-II after the vertex detector upgrades, when relative abundances of the different charmed hadron states will be measured. Also, direct photon yields and photon Hanbury-Brown-Twiss (HBT) interferometry measurements at different transverse momenta can put additional constraints on models since they measure the space-time properties of the system as it evolves, even before equilibration. In particular, photon $\mathrm{HBT}$ at $\mathrm{p}_{\mathrm{T}}>2 \mathrm{GeV} / \mathrm{c}$ (mostly from hard scattering) would establish the initial size. However, the signal (effective amplitude of the HBT correlation) is only a few parts per thousand, requiring statistics (and thus luminosity) well beyond current RHIC capabilities, but within the capabilities of RHIC II.

Photon elliptic flow is another independent test of thermalization after the parent processes have been properly disentangled. If (and since) the photons from the initial scattering do not interact with the medium, their $v_{2}$ is expected to be zero. However, photons are not only produced in the initial hard scattering, but may also originate from jet partons scattering from thermal partons (jetthermal interaction) or from Brehmsstrahlung produced in scattering from a quark. These photons are expected to exhibit a negative $\mathrm{v}_{2}$ since more material is traversed out-of-plane (which is the major axis in coordinate space). On the other hand, photons from thermal radiation should reflect the dynamical evolution of the hot and dense matter with positive $v_{2}$. These measurements will require high luminosity.

\subsubsection{Timescale for thermalization}

The thermalization process is difficult to model theoretically due to its highly non-equilibrium nature. An important step in understanding the thermalization mechanism is to determine experimentally the timescale on which thermalization occurs. One way to do that is to compare a dynamical macroscopic model of the system evolution to experimental data and then infer the timescale of thermalization from the model.

Since the hydrodynamic approach is a macroscopic description of the system, it is not applicable to describe the pre-equilibrium stage of the reaction and can not give an answer on how thermal equilibrium is achieved. It can, however, give an estimate of the time it takes for the system to reach local thermal equilibrium by connecting the measured elliptic flow and the evolution of the anisotropic pressure gradients in the system. The success of ideal hydrodynamics in describing the large elliptic flow observed at RHIC requires very short thermal equilibration times, of the order $\tau_{\text {therm }}<1 \mathrm{fm} / \mathrm{c}$. In fact, at least one model that reproduces thermal photon rates measured in PHENIX (and includes jet-plasma interactions as well) assumes thermalization in $0.33 \mathrm{fm} / \mathrm{c}$. Finding the mechanism that equilibrates the system so quickly remains one of the major challenges for RHIC-II.

\subsubsection{Thermalization mechanism and links to experiment}

The thermalization process itself can only be assessed using a microscopic description of the preequilibrium system dynamics. Microscopic transport models need experimental input to constrain the transport coefficients in the medium. Heavy quarks are a good probe of the transport properties of the medium. Due to the large c-quark mass, the charm equilibration time is expected to be about 
a factor of 5 larger than the the light quark equilibration time. Theoretically, the charm diffusion coefficient has been related to the charm energy loss and momentum broadening. Utilizing transport theory, these quantities can then be used as an input for the calculation of charm elliptic flow, thus creating a relation between an experimentally accessible quantity and the diffusion coefficient.

Measurements of open charm production and flow data in heavy ion collisions are challenging due to the small production cross-sections and the experimental limitations on charm particle identification. While the $\mathrm{R}_{\mathrm{AA}}$ measurement from charm semileptonic decay data already has discriminative power, the statistical errors on the $\mathrm{v}_{2}$ results will have to be greatly improved to allow definitive tests of models. This will be achieved by a combination of detector upgrades and the RHIC II luminosity upgrade. Specifically, the vertex detector upgrades in PHENIX and STAR will make possible direct charm and beauty identification over a broad kinematic range with reduced backgrounds, and reaction plane resolution will improve by as much as a factor of two due to improved kinematic coverage.

\subsubsection{Viscosity}

The important discovery that the hot matter created in the early stage of heavy-ion collisions at RHIC flows with less viscosity than any previously known fluid poses a new challenge: to measure and calculate the actual (small) value of the shear and/or bulk viscosity coefficients for the quarkgluon plasma. Intellectually this is an exciting problem since the physics of strongly coupled plasmas and the question how to describe them theoretically transcends traditional disciplinary boundaries. The phase structure of strongly coupled many-body systems is an important issue also for very dense electromagnetic plasmas, both in the laboratory and in astrophysical environments, it plays a key role in understanding the BEC-BCS crossover in systems of cold trapped fermionic atoms, and it has found the interest of particle theorists who discovered that the tools of superstring theory can be successfully applied to the calculation of transport coefficients in systems with asymptotically large (infinite) coupling strength. Based on these calculations, one now expects that there exists a universal lower limit for the ratio of shear viscosity to entropy density, $\eta / \mathrm{s}$, and that the QGP viscosity may even approach this lower limit.

An experiment-driven approach to extracting the viscosity of the fireball matter at RHIC requires a detailed comparison of measured collective flow patterns with a viscous relativistic hydrodynamic framework which is presently being developed. The flow patterns to be studied include $v_{0}$ (the isotropic radial flow) and the anisotropic higher order flow terms $\mathrm{v}_{1}$ (directed flow), $\mathrm{v}_{2}$ (elliptic flow) and $v_{3}$. It will be necessary to measure $v_{0}, v_{1}$ and $v_{2}$ at all rapidities in collisions between equal nuclei, and $v_{1}$ and $v_{3}$ at midrapidity in collisions between unequal nuclei. Direct comparisons between data and microscopic transport simulations, followed by a theoretical extraction of the viscosity coefficients from the latter, will be helpful, too. In order to fully constrain the hydrodynamic model and to separate viscous effects from those generated by variations of the initial state and the Equation of State, a complete flow analysis of all hadrons as well as direct photons must be performed, for several (symmetric and asymmetric) collision systems at various collision energies. This requires high-statistics transverse momentum spectra and anisotropic flow measurements for a large number of identified hadrons with different masses and quark contents. Elliptic flow of multi-strange baryons and mesons such as the $\Omega, \Xi$ and $\varphi$, which are thought to be less strongly coupled to the hadronic fluid than pions, kaons and nucleons, 
should help to separate late hadronic dissipation effects from viscous effects during the early quark-gluon plasma stage. Since the heavy charmed quarks are expected to be less well thermalized than lighter quarks, the elliptic flow of charmed hadrons should yield valuable information about the microscopic thermalization mechanisms and the origin of quark-gluon plasma viscosity. To perform all these measurements, data of statistical quality similar to Run 4 must be collected for each of a dozen or so combinations of collision system and beam energy. This can only be realized in an acceptable time period once the upgraded capabilities at RHIC II, both in collider luminosity and increased data acquisition rate, become availabe. 


\section{Do Heavy Quarks Flow?}

The initial time $t_{0}$ when local equilibrium is reached and hydrodynamics becomes applicable has been treated as a free parameter in all elliptic flow calculations to date. To obtain a precise measure of the viscosity of the matter created at RHIC, we need an accurate determination of $t_{0}$. Heavy quarks require many more collisions to thermalize than light quarks, so their elliptic flow is

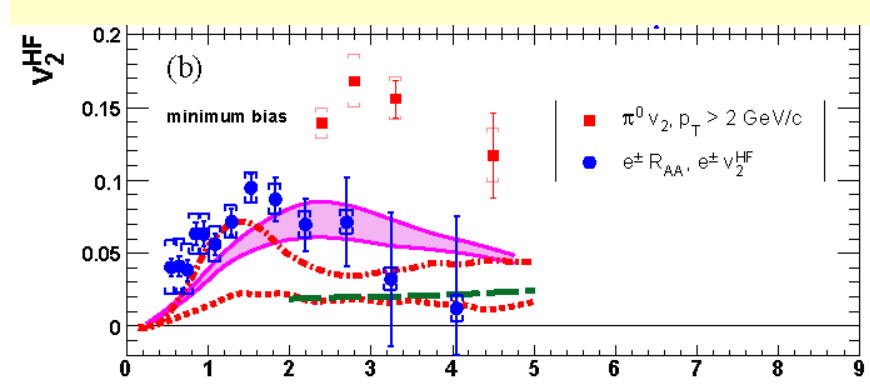

$200 \mathrm{GeV} A u+A u$ collisions, measured by PHENIX. a valuable chronometer to measure $t_{0}$. Figure 1 shows our current knowledge of heavy quark elliptic flow together with several model predictions.

PHENIX and STAR plan complementary upgrades to obtain far more precise results at RHIC II. The PHENIX Silicon Vertex Detector upgrade will dramatically reduce backgrounds by tagging electrons as arising from decays of $D$ or $B$ mesons. The STAR

Heavy Flavor Tracker upgrade will provide direct topological reconstruction of heavy flavor decays, such as $D \rightarrow K \pi$, on an event-by-event basis. Figures 2 and 3 show the precisions that are expected from a typical $\mathrm{Au}+\mathrm{Au}$ run at RHIC II luminosity.

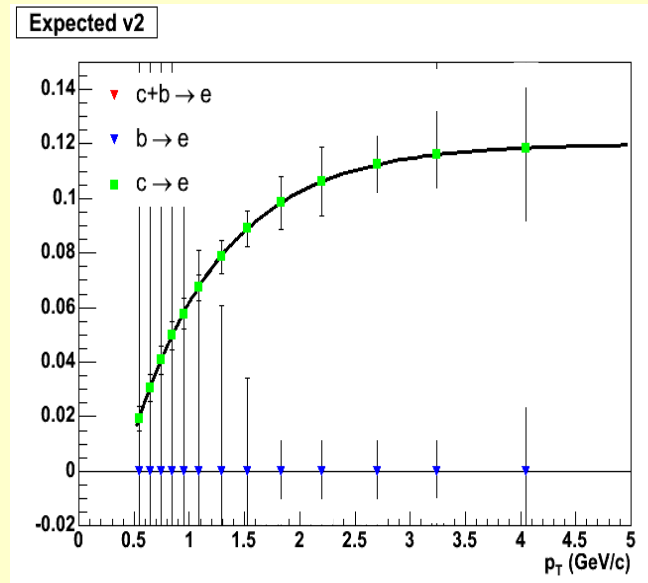

Figure 2: PHENIX sensitivity to elliptic flow of electrons from charm and bottom decays at RHIC II.
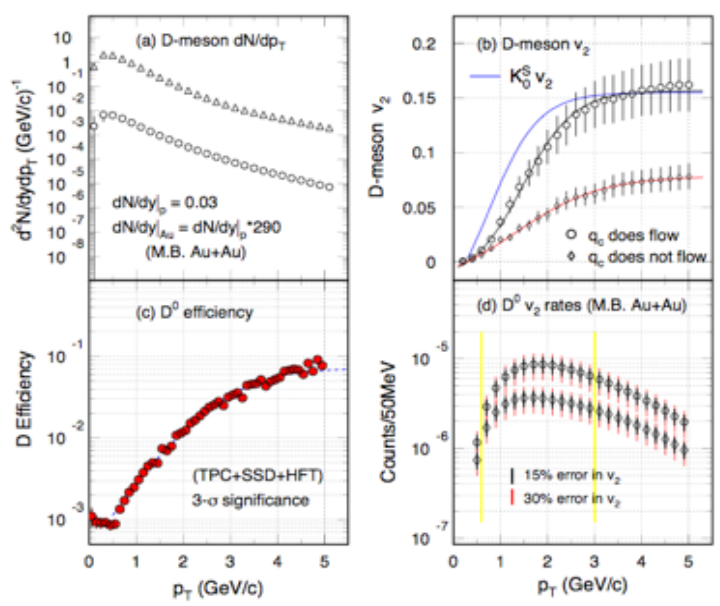

Figure 3: STAR sensitivity to elliptic flow of D mesons at RHIC II, compared to model predictions that assume either charm flows like a light quark or charm doesn't flow. 


\subsubsection{The equation of state}

For a relativistic fluid, the equation of state is specified by the temperature and the energy density (or entropy density). The pressure is not an independent intensive variable, because it adjusts to changes in the other state variables by particle production and annihilation. Thus independent measurements of the energy density and temperature are needed to characterize the matter formed at RHIC. In section 5.1.2.1 we discuss measurements of the energy density. Then in section 5.1.2.2 we describe measurements aimed at determining the temperature, and discuss how the temperature and energy density measurements might be used to infer the number of degrees of freedom.

\subsubsection{Measurements of energy density}

In heavy-ion collisions, there are a number of independent techniques to determine the initial energy density. The most straightforward method relates the observed final-state rapidity density of transverse energy, $\mathrm{dE}_{\mathrm{T}} / \mathrm{d} \eta$, to the initial energy density via the Bjorken model. This assumes boost invariant hadron spectra in the midrapidity region, and requires independent knowledge of the thermalization time, which can be estimated for example from the study of elliptic flow. Such estimates provide a lower bound on the initial energy density since they neglect work performed during longitudinal expansion. The consensus value that resulted at RHIC was $\varepsilon>5 \mathrm{GeV} / \mathrm{fm}^{3}$ at an initial time $\tau_{0} \sim 1 \mathrm{fm} / \mathrm{c}$, well above the expected transition energy density of approximately 0.7 $\mathrm{GeV} / \mathrm{fm}^{3}$.

The suppression of high $\mathrm{p}_{\mathrm{T}}$ hadron production, often referred to as "jet quenching", provides an alternative determination of the initial energy density. Jet quenching arises when energetic partons lose energy while propagating through the dense medium. It has been argued that this energy loss is dominated by gluon radiation induced by parton multiple scattering in the dense matter, in which case the energy loss is proportional to the gluon density of the medium.

Experimental data from RHIC [for example, Fig. 2] show the yields of high $\mathrm{p}_{\mathrm{T}}$ single hadrons are suppressed by factors of 4-5 in central $\mathrm{Au}+\mathrm{Au}$ collisions at ${\sqrt{\mathrm{S}_{\mathrm{NN}}}}=200 \mathrm{GeV}$. Back-to-back dihadron azimuthal correlations, which are characteristic of dijets, are also strongly suppressed. Perturbative QCD models based on parton energy loss and modified jet fragmentation functions have been very successful in describing these jet quenching observations. Phenomenological studies of the high $\mathrm{p}_{\mathrm{T}}$ results deduce an initial gluon density of about $30 / \mathrm{fm}^{3}$ at an initial time $\tau_{0} \sim$ $0.2 \mathrm{fm} / \mathrm{c}$. This corresponds to an initial energy density of $\varepsilon \sim 15 \mathrm{GeV} / \mathrm{fm}^{3}$, about 100 times the energy density in cold nuclear matter or 20 times the expected transition energy density.

Studies of jet quenching and extraction of energy density have so far relied on the assumption of the dominance of gluon radiation, which is one of the uncertainties in the theory. Theoretical uncertainties are still large due to the neglect of elastic energy loss and the complication of trigger bias associated with the measurement of single inclusive spectra. Future measurements using direct $\gamma$-jet correlations and heavy flavor tagging will provide much needed improvements.

Gamma-jets: Gamma+jet events have long been considered the "golden probe" for directly measuring the energy loss of hard-scattered partons propagating through the medium. Direct photons originate from an initial hard scattering. In direct $\gamma$-jet events, the photon provides a 
determination of the energetic parton transverse momentum before it interacts with the dense medium. The away-side jet can then be reconstructed to characterize both the partonic energy loss and the response of the medium to the deposited energy and momentum.

The challenge in selecting these $\gamma$-jet events is separating the direct photons from those that come from hadronic decays. Experimentally, there are two possible methods to make such a measurement. One method is to measure both the correlation between $\pi^{0}$ and jets and the correlation between inclusive photons and jets. The inclusive photons include those photons from hadronic decays (mostly $\pi^{0} \rightarrow \gamma+\gamma$ ). From the measurement of the $\pi^{0}$-jet correlation and a simulation of the $\pi^{0}$ decay, one can statistically subtract the jet correlation due to the $\pi^{0}$ from the inclusive $\gamma$-jet correlation. A second method is to isolate the pure direct $\gamma$ with veto-cuts that minimize the contribution of photons from $\pi^{0}$ and $\eta$ decays. The second method is more statistically limited than the first, due to the large efficiency losses necessary to achieve high purity in the direct photon sample. However, as measured by PHENIX, the fraction of direct photons is dramatically increased in central $\mathrm{A}+\mathrm{A}$ collisions due to the suppression of pions from jet fragmentation. At $\mathrm{p}_{\mathrm{T}} \sim$ $8 \mathrm{GeV} / \mathrm{c}$, approximately $60 \%$ of all photons are direct photons in the most central $(0-10 \%)$ events. Based on a PHENIX analysis of Run $4 \mathrm{Au}+\mathrm{Au}$ data, and assuming 20 times the Run 4 statistics at RHIC II, there will be approximately 10k direct photons with a purity of $60 \%$ for $\mathrm{p}_{\mathrm{T}}>8 \mathrm{GeV} / \mathrm{c}$, and $3 \mathrm{k}$ direct photons with a purity of $80 \%$ for $\mathrm{p}_{\mathrm{T}}>10 \mathrm{GeV} / \mathrm{c}$.

Jet quenching and multiple particle correlations: More differential information is provided by multi-hadron correlations within a pair of jets. If the suppression of leading high $\mathrm{p}_{\mathrm{T}}$ hadrons is caused by parton energy loss, the study of intermediate energy and soft hadrons associated with a quenched jet can shed light on the distribution of radiated gluons and, more importantly, the response of the medium to the jets. Recent experimental studies of angular correlations of soft hadrons with respect to a quenched jet have revealed an unexpected structure. In central $\mathrm{Au}+\mathrm{Au}$ collisions, soft hadrons associated with a quenched jet (in the opposite direction from a trigger hadron) are peaked at a finite angle $\Delta \Phi \sim 1$ radian away from the jet, whereas they peak in the direction of the jet in peripheral $\mathrm{Au}+\mathrm{Au}$ or $\mathrm{p}+\mathrm{p}$ collisions. This observation has led to suggestions of different scenarios for the interaction between jets and the dense medium, ranging from propagation of sound waves excited by the fast jets to Cerenkov gluon radiation from jets and deflection of jets off the surface when they propagate tangentially. The only way to elucidate the original mechanism for the observed two-hadron correlation is through multi-hadron correlations. If confirmed by detailed study of multi-hadron correlations, this could directly measure the sound velocity or the dielectric constant of the medium.

Another interaction between energetic jets and the bulk matter is recombination of jet partons with thermal partons in the medium. Recombination will modify the hadronization mechanism for jet fragmentation in the medium. Identified particle di-hadron and multi-hadron correlations will quantify this modification and shed light on the effective degrees of freedom of the medium just before hadronization. The enhanced particle identification provided by the planned PHENIX and STAR detector upgrades will make such studies practical over the entire intermediate $\mathrm{p}_{\mathrm{T}}$ region, where parton recombination and coalescence have been invoked to explain the enhanced baryon to meson ratio and constituent (ie. valence) quark scaling of elliptic flow.

Current three-hadron analyses are limited by the number of such events sampled with triggered 
high $\mathrm{p}_{\mathrm{T}}$ hadrons and also by the $\mathrm{p}_{\mathrm{T}}$ range of the correlated hadrons. Experiments at RHIC II with increased luminosity and upgraded detectors can dramatically improve upon the present experiments. Furthermore, within a sample of direct $\gamma$-jet events, one can eliminate complicated geometrical distributions associated with high $\mathrm{p}_{\mathrm{T}}$ hadron-triggered jets. Di-hadron angular correlations in the opposite direction from the direct $\gamma$ can unambiguously test geometric effects which could lead to deflection of jets near the surface of the dense matter.

Heavy quarks and associated jets: The same pQCD parton energy loss models can be applied to predict the suppression of heavy flavor meson spectra. Heavy quarks lose less energy via gluon radiation than light quarks do because of the dead-cone feature in the gluon radiation spectra. The mass dependence of the gluon formation time also changes the length dependence. First open charm spectra in heavy-ion collisions at RHIC have been obtained via the measurements of nonphotonic single electron spectra, assuming the dominant contributions come from leptonic decays of $\mathrm{D}$ and $\mathrm{B}$ mesons. The observed suppression of single electron spectra is surprisingly large, as large as the suppression of pions from the fragmentation of light quarks. This observation so far still remains a puzzle. Different theoretical studies taking into account contributions from D and B meson decays have failed to describe the data, even when including elastic energy loss for heavy quarks. The obvious approach to solve the puzzle is to measure the D meson spectra directly through $\mathrm{D} \rightarrow \mathrm{K}+\pi$ and the $\mathrm{B}$ meson spectra through $\mathrm{B} \rightarrow \mathrm{J} / \psi+\mathrm{X}$ with $\mathrm{RHIC}-\mathrm{II}$ and the upgraded experiments.

According to QCD, gluons with their larger color charge experience over twice the energy loss of quarks. Flavor-tagged jets provide an excellent way to observe this effect directly. Measurements of $\mathrm{D}$ meson suppression and D-meson tagged jets will provide direct access to a clean heavy quark sample. Jets with leading $\mathrm{J} / \psi$ (hidden charm) particles come primarily from gluon jets, where the $\mathrm{J} / \psi$ is produced promptly, or bottom-quark jets where a B-meson decays into a $\mathrm{J} / \psi$ after propagating typically for a fraction of a millimeter. In contrast, light-hadron jets arise from a mixture of leading gluons and light quarks. Thus, we can study the properties of jet samples containing different compositions of light quark, heavy quark, and gluon jets to isolate their different interactions with the dense medium.

The LHC will provide a higher jet production rate and a bigger reach in transverse momentum than is available at RHIC energies. The lower rate of jet production at RHIC will be advantageous in some ways, however. The energy loss is larger relative to the energy of the jet for the lower jet energies at RHIC, and the lower rate of jet production at RHIC translates into smaller background for the study of multi-hadron correlations. In addition, high transverse momentum measurements are not only probing the effect of energy loss per se, but also the coupling of the jets to the matter. At RHIC, the intermediate $\mathrm{p}_{\mathrm{T}}$ region shows evidence for a novel hadronization mechanism, which can be further investigated through correlations between high $\mathrm{p}_{\mathrm{T}}$ and intermediate $\mathrm{p}_{\mathrm{T}}$ particles. Thus, RHIC is an ideal place to study the collective response of the medium when excited by the propagating jets.

Several of the measurements discussed above, especially the gamma-jet and flavor tagged-jet measurements, require the large statistics data sets that will be available after the RHIC II luminosity upgrade. Furthermore, most of the measurements, particularly those involving heavy flavor, will require planned detector upgrades, including the PHENIX Silicon Vertex Tracker and 
the STAR Heavy Flavor Tracker and DAQ1000 data acquisition upgrade. In addition, the gammajet measurements will benefit from the additional calorimetry that is planned for the forward rapidity region, the PHENIX Nosecone Calorimeter and the STAR Forward Meson Spectrometer.

\subsubsection{Initial temperature: how to measure and use it ?}

Thermal emission rates for electromagnetic radiation can in principle be used to "infer" the temperature of thermalized matter in heavy-ion collisions if (i) the emission strength represented by the electromagnetic spectral function is reasonably well determined, and (ii) a kinematic window can be identified where radiation from a reasonably well-defined temperature regime prevails. Note that photons (unlike hadrons) provide direct information from before the kinetic freeze-out of hadrons. On the other hand the same property makes interpretation of photon temperatures more difficult, since the photon spectral shapes and yields will be a convolution of many processes over the lifetime of the system. However, three regimes emerge where QGP radiation outshines both hadron gas and primordial emission:

3-momentum integrated dilepton spectra at intermediate mass, $\mathrm{M} \sim 1.5-3 \mathrm{GeV}$;

direct photon spectra at intermediate $q_{t} \sim 1.5-3 \mathrm{GeV}$;

- low-mass dilepton spectra at transverse momenta above $q_{t} \sim 2 \mathrm{GeV}$ ("low virtuality" photons).

Here $q_{t}$ is the transverse momentum of the real or virtual (dilepton pair) photon. In practice a careful assessment of additional sources, including "pre-equilibrium" contributions and jet-plasma interactions, is mandatory before firm conclusions on the "thermal" component can be drawn. On the other hand jet-plasma interactions themselves give important information on energy density.

The measurement of the slope of the resulting thermal photon spectrum, $\mathrm{T}_{\text {eff, }}$ does not yet directly reflect the temperature of the hot matter, as photons are emitted throughout the space-time volume of the evolving matter implying varying temperatures, as well as blue shifts due to collective expansion. Nevertheless, a correlation between the apparent photon slope and the (maximum) temperature attained in the system persists. The measured $\mathrm{T}_{\text {eff }}$ then provides an empirical link to the effective number of degrees of freedom of the system via $d_{o f}=\left(30 / \pi^{2}\right) \varepsilon / T_{\text {eff }}{ }^{4}$ or $d_{o f}=\left(45 /\left(2 \pi^{2}\right)\right)$ $\mathrm{s} / \mathrm{T}_{\text {eff }}{ }^{3}$.

The initial (maximal) energy or entropy density $(\varepsilon, s)$ are difficult to access experimentally. Yet, information on the temperature dependence of $d_{\text {of }}$ can be obtained via centrality and ${\sqrt{\mathrm{S}_{\mathrm{NN}}}}$ dependencies of $\mathrm{T}_{\text {eff }}$ and the observables related to $\varepsilon$ and $\mathrm{s}$. At the minimum, it has been argued that it is possible to discriminate a QGP-like equation of state with fixed $d_{o f}$ above $\mathrm{T}_{\text {eff }}$ from a hadronic resonance gas with rapidly rising number of degrees of freedom, by establishing the dependence of $\mathrm{T}_{\text {eff }}$ on the pseudorapidity density of the charged particles. As a further consistency check, one can relate the degrees of freedom to $d_{\text {of }} \alpha \mathrm{s}^{4} / \varepsilon^{3}$.

While a rapid thermalization of the matter at full RHIC energy is fairly well established, its composition in terms of quark and gluon degrees of freedom (chemical equilibration) is much less clear (as are the equilibration mechanisms themselves). From the prevalence of gluons in the relevant $\mathrm{x}$-range of the incoming nuclei one might expect the early matter to be a gluon plasma 
(GP). However, recent calculations of quark-antiquark pair production within the classical fields generated by the incoming Au nuclei indicate a rather fast approach to chemical equilibrium. This, in turn, would have important consequences for disentangling the relevant mechanism(s) for energy loss of fast partons. The apparently obvious way to test these questions is by dileptons, which maximally distinguish between gluons and quarks (with zero, $1 / 3$ or $2 / 3$ electric charges, respectively). Identifying a GP (or more generally, the $d_{o f}$ above $\mathrm{T}_{\text {crit }}$ ) from thermal dileptons will have to involve a quantitative assessment of both slope and absolute magnitude of the thermal spectrum, after "removal" of non-thermal sources including Drell-Yan, and, more importantly, correlated open-charm decays as well as pre-equilibrium and jet-plasma interactions. Note that at LHC gluons will dominate whereas at RHIC both quarks and gluons will be accessible with electromagnetic probes making the LHC and RHIC measurements complementary.

Dilepton emission subsequent to the initial (hard) N-N collisions but before the assumed thermalization time (the so-called pre-equilibrium contribution) can be addressed in a parton cascade approach. Once the Landau-Pomeranchuk-Migdal (LPM) interference effects are accounted for, a recent calculation indicates that the combined initial pQCD plus pre-equilibrium yield is not very different from the pQCD contribution alone, with the thermal yield taking over for momenta below $q_{t} \approx 2.5 \mathrm{GeV}$ or so. The implicit present model dependencies will have to be further reduced to achieve enough sensitivity to discern the composition of the early matter.

We conclude that the three most promising regimes of thermal QGP radiation for inferring the earliest temperatures in the system are: (i) intermediate-mass dileptons, (ii) photons and (iii) lowmass dileptons at intermediate transverse momentum $\left(q_{t} \sim 2-4 \mathrm{GeV}\right)$. These measurements could be augmented by a $\gamma-\gamma$ correlation analysis which provides complementary temperature and fireball size information on the early stages. Note that the RHIC environment is ideal for the dilepton measurements described above, since the irreducible background from open charm is still manageable at RHIC energies.

To achieve the required background rejection and high precision charm measurements, PHENIX needs the Hadron Blind Detector and vertex detector, and STAR needs the Time-of-Flight detector, Heavy Flavor Tracker and an updated Data Acquisition System. The study of direct photon HBT correlations is a feasible experiment with STAR given DAQ 1000 and an improved calorimeter. Initial measurements of dileptons will be accomplished after those new detectors are in place. However, energy scans with high statistics require luminosity upgrades, and so does high $\mathrm{p}_{\mathrm{T}}$ photon $\mathrm{HBT}$. 


\section{Jets at RHIC II and LHC}

Correlations of two or more high momentum particles arising from jet fragmentation probe the mechanism of energy loss. This physics is probed with jets of energy $10 \mathrm{GeV}$ or higher. The correlation of one hard particle $\left(>5 \mathrm{GeV} / \mathrm{c} \mathrm{p}_{\mathrm{T}}\right)$ with one or more softer particles $\left(\mathrm{p}_{\mathrm{T}}=1-4 \mathrm{GeV} / \mathrm{c}\right)$ from the opposing jet probes the response of the medium to the energy deposited by a jet traversing it. The "golden channel" for jet probes of the hot, dense medium is correlations between $\gamma$-q pairs arising from QCD Compton scattering; the direct photon tags the jet energy, making it possible to quantify the lost energy and its fate in the medium.

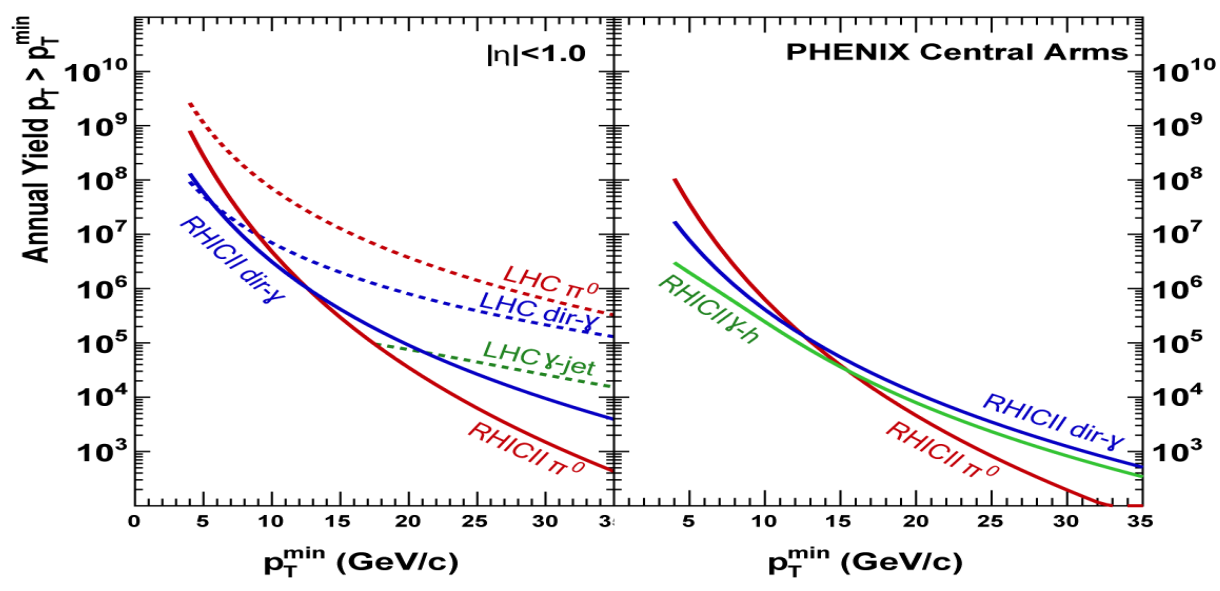

The figure shows the annual recorded number of events with neutral pions, direct photons, and photon-jet coincidences at RHIC II and LHC for $\mathrm{Au}+\mathrm{Au}$ or $\mathrm{Pb}+\mathrm{Pb}$ collisions, where the trigger particle has $\mathrm{p}_{\mathrm{T}}>\mathrm{p}_{\mathrm{T}}{ }^{\mathrm{min}}$. The left hand side shows the annual yield into rapidity $-1<\mathrm{y}<1$ (STAR and ALICE), the right hand side provides the yield into the PHENIX central arms.

Results for single direct $\gamma$ and $\pi^{0}$ are solid lines for RHIC II and dashed lines for LHC. Two striking results are immediately apparent. First, at RHIC direct photons dominate over decay photons above $\sim 12 \mathrm{GeV} / \mathrm{c} \mathrm{p}_{\mathrm{T}}$, greatly simplifying $\gamma$-jet measurements. Second, using 1000 counts as a useful minimum, we see that direct photons can be measured to $\mathrm{p}_{\mathrm{r}}>30 \mathrm{GeV} / \mathrm{c}$ at RHIC II.

Yields where hadrons of $\mathrm{p}_{\mathrm{T}} \geq 4 \mathrm{GeV} / \mathrm{c}$ are detected in coincidence with direct photons are labeled $\gamma$-h. The figure shows that RHIC II will measure direct photon-hadron coincidences for $35 \mathrm{GeV} / \mathrm{c}$ photons. The PHENIX central arms alone will access $\gamma$-h for $30 \mathrm{GeV} / \mathrm{c}$ photons, though upgrades underway will increase the PHENIX acceptance nearer to that in the left panel.

The LHC " $\gamma$-jet" curve shows yields for fully reconstructed away side jets. Because the $\gamma / \pi^{0}$ ratio remains below 1.0 , even at $\mathrm{p}_{\mathrm{T}}=35 \mathrm{GeV} / \mathrm{c}$, a larger number of events will be required at the LHC in order to enable statistical subtraction of decay photon-jet correlations. At RHIC, full jet reconstruction is not available for the relatively low energy jets measured thus far, but attempts are currently underway. 


\subsubsection{Exploring the QCD phase diagram}

The discussion so far has focused mainly on what can be learned about the properties of the QGP at $\mathrm{T}>\mathrm{T}_{\mathrm{c}}$ and low baryon chemical potential in heavy ion collisions. But a detailed understanding of the phase structure of QCD requires systematic exploration of the phase diagram over a range of temperature and baryon chemical potential. Two aspects of that systematic exploration are discussed here. We start by discussing the search for the QCD critical point and the subsequent appearance of a first order phase transition. Then we discuss the planned programs for studying the expected modifications to hadron properties in the region of the phase transition to a QGP.

\subsubsection{Search for the QCD critical point}

There are strong theoretical reasons for believing that the QCD phase diagram contains a line of first order phase transitions, starting at large baryon chemical potential and low temperature, and terminating at a critical point as shown in Fig. 10.

Very briefly, the argument is as follows: Lattice gauge calculations at vanishing baryon chemical potential, and with physical masses for the $\mathrm{u}, \mathrm{d}$ and s quarks, suggest that the phase transition is a rapid crossover in a narrow temperature range at about $170 \mathrm{MeV}$, without the singularities in the bulk thermodynamic properties that would be present for a first order phase transition. At low temperature and high baryon chemical potential, however, many QCD motivated calculations produce a strong first order phase transition with a phase coexistence region. These two limiting cases suggest that there exists a critical point (a second order phase transition) at the end of a line of first order phase transitions.

The location of the critical point still has large theoretical uncertainties, but the available estimates indicate strongly that it is within the part of the phase diagram that can be probed by heavy ion collisions at RHIC or at the lower energy FAIR facility at GSI. The key control parameter is the baryon density, which may be increased by lowering the collision energy and/or by measuring at forward rapidities. Even higher baryon densities may be achieved by applying these same techniques to colliding beams of uranium provided by the EBIS ion source currently under construction. The experimental program would require varying the baryon chemical potential and looking for signatures of the critical point, or of a first order phase transition, as the trajectory of the collision in QCD phase space passes below the critical point. The critical point is a true thermodynamic singularity at which the quark number susceptibilities diverge and the order parameter fluctuates on long wavelengths. The simplest proposed observables are event by event fluctuations of the mean transverse momentum and of the total charge multiplicity per event. It is expected that as the control parameter is varied, these signatures would strengthen and then weaken as the critical point is approached and then passed.

There is already considerable experimental data spanning ${\sqrt{\mathrm{S}_{\mathrm{NN}}}}=2.7 \mathrm{GeV} / \mathrm{c}$ to $200 \mathrm{GeV} / \mathrm{c}$ from the AGS, SPS and RHIC programs. Some interesting structure has been observed in the energy dependence of, most notably, the strangeness-to-pion ratio at SPS energies, and it has been suggested that this is an indication of deconfinement. Various studies have been performed searching for event by event fluctuations in apparent temperature, mean transverse momentum, multiplicity, net charge and momentum correlations, all with no indication of the large signals 
expected near the critical point. However the range of baryon chemical potential from about 70 to $240 \mathrm{MeV}$ remains unexplored, and there are estimates suggesting that low ${\sqrt{\mathrm{S}_{\mathrm{NN}}}}_{\mathrm{RHIC} \text { running }}$ could significantly improve the statistical and systematic errors even where there are existing data at SPS energies. Finally, the advantages of being able to make comparative measurements over a range of energies in collider geometry, and with the same detectors, are large because most systematic effects remain constant, in contrast to the situation for fixed-target measurements.

The feasibility of this program depends very strongly on the capability of RHIC to provide

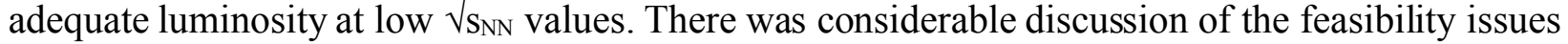
at a workshop held at BNL on March 9-10, 2006 (see "Can we discover the QCD critical point at RHIC?" https://www.bnl.gov/riken/QCDRhic). It was concluded that there are no apparent "show stoppers" for operation of RHIC at 5-50 GeV. This would extend the RHIC baryo-chemical potential range to $30-500 \mathrm{MeV}\left({\sqrt{\mathrm{S}_{\mathrm{NN}}}}\right.$ from $5 \mathrm{GeV}$ to $\left.200 \mathrm{GeV}\right)$. The possibility of using electron cooling at low energies to greatly increase the luminosity is being pursued. The baryon chemical potential coverage at FAIR will be approximately $400-800 \mathrm{MeV}$.

\subsubsection{Medium effects on properties of hadrons}

The generation of hadronic masses is a key problem in QCD and intimately related to the underlying structure of the vacuum. At zero temperature and baryon density, the presence of quark and gluon condensates is believed to create the "constituent" quark mass of $300-400 \mathrm{MeV} / \mathrm{c}^{2}$, and therefore to be at the origin of the masses of light hadrons (which, in turn, account for $\sim 99 \%$ of the visible mass in the universe). At the same time, the so-called chiral symmetry of QCD (which for massless quarks conserves right- and left-handed "chirality" states) is spontaneously broken. At the hadronic level, chiral symmetry breaking entails the splitting of spectral functions within chiral multiplets, such as $\pi-\sigma, \rho-a_{1}$ and $N-N^{*}(1535)$, creating mass gaps of typically $\sim 500 \mathrm{MeV} / \mathrm{c}^{2}$. At the critical temperature, $\mathrm{T}_{\mathrm{c}} \sim 170 \mathrm{MeV}$, lattice calculations indicate that chiral symmetry becomes restored, forcing chiral partners (multiplets) to become degenerate. Dilepton invariant-mass spectra are a unique observable to measure in-medium spectral functions of low-mass vector mesons, most notably of the $\rho$ meson, and thus probe changes in the underlying vacuum structure, especially the approach to chiral symmetry restoration. In addition, at higher invariant mass, there exists the possibility to detect putative resonant quark-antiquark states above $T_{c}$, which would give direct evidence of a strongly interacting QGP (sQGP) and reveal the scale for the thermal parton mass.

At the SPS, dilepton measurements have triggered vigorous theoretical activity; recent precision data by NA60 have enabled substantial progress in discriminating different in-medium effects on the $\rho$-meson spectral function, but open questions remain concerning possible scenarios of chiral symmetry restoration. Nuclear collisions at RHIC energies provide the unique opportunity to establish quantitative connections with lattice QCD calculations, since both predominantly operate at (almost) vanishing baryon chemical potential and the irreducible backgrounds from open charm are small enough to allow precision measurements.

Low-mass (axial-) vector meson spectroscopy close to the chiral transition: Precision dilepton data will provide detailed information on medium modifications of $\rho, \omega$ and $\varphi$ mesons, and thus illuminate the question of hadronic mass generation. "Quality control" of theoretical models via independent constraints from symmetries, QCD sum rules and phenomenology is essential to limit 
the scope of viable axial-/vector spectral functions. An excitation function will allow to discriminate effects of temperature and (net-) baryon density and thus systematically map out inmedium effects across a significant part of the QCD phase diagram. A convincing deconvolution of the vector spectral functions from the measured spectra will require systematic variations of system sizes via centrality and species scans, coupled with reliable space-time descriptions to emerge from progress in hydrodynamic and transport simulations.

A promising option to experimentally infer the approach to chiral symmetry restoration is the use of novel measurements of the axial vector spectral function via $\pi \gamma$ invariant-mass spectra. While the pion is subject to final-state absorption, the accessible density of the medium in $\pi \gamma$ spectra will be higher than in previously measured $\pi \pi$ invariant-mass spectra. On the theoretical side, chiral effective models can establish direct links between dilepton observables (i.e. vector spectral functions) and order parameters computable in lattice QCD. The key tools are chiral sum rules which relate energy-weighted moments of "vector minus axial vector" spectral functions to the pion decay constant and quark condensates at finite temperature. Unquenched lattice QCD evaluations of these quantities, as well as realistic calculations of vector and axial vector spectral functions, are therefore to be pursued with high priority.

Footprints of chiral symmetry restoration are furthermore expected at dilepton masses around the $\mathrm{a}_{1}$ mass (1-1.5 GeV), due to "chiral (vector-axial vector) mixing". To detect the pertinent continuum enhancement by a factor of $\leq 2$ requires accurate charm and background determination, providing a signal with no more than $20 \%$ total error. This background measurement can be achieved with the planned vertex detector upgrades, in combination with sufficient statistics.

QGP resonances: The only way of directly testing the exciting possibility of partonic bound states/resonances in a sQGP experimentally is to search for a resonant dilepton signal. The largest sensitivity for the discovery of pertinent vector states is in the $\mathrm{M}=2 \mathrm{GeV}$ mass region, which, in fact, coincides with current expectations from lattice QCD and effective models. RHIC energies provide optimal conditions for this search as the initial temperatures are close to the anticipated dissolution temperatures of the bound states. As is the case for signatures of chiral mixing at lower mass, an accurate determination of background and charm sources is mandatory to restrict total experimental errors to below the $\sim 30 \%$ level. If resonance states exist at masses below $\sim 1.5 \mathrm{GeV}$, their discrimination from chiral mixing effects will be more involved, increasing the demand for accuracy and for systematic centrality and excitation-function studies.

To achieve the required background rejection and high precision charm measurements, PHENIX needs a Hadron Blind Detector and vertex detector, and STAR needs a Time-of-Flight, Heavy Flavor Tracker and upgraded Data Acquisition System. Initial measurements of dileptons will be accomplished after these new detectors are in place. However, an energy scan with high statistics comparable to recent NA60 data requires the RHIC luminosity upgrade.

\subsubsection{Deconfinement}

Two ways have been suggested so far to demonstrate experimentally that deconfinement has occurred. One is to determine the number of degrees of freedom from the measured temperature and the energy density. This is discussed in section 5.1.2. The other is to experimentally verify that 
states do in fact disappear at temperatures for which lattice calculations indicate that they are no longer well defined. For the latter, we look to quarkonium (charmonium $-\mathrm{J} / \psi, \chi_{\mathrm{c}}$ and $\psi^{\prime}$ - and bottomonium - Y(1S), $\chi_{b 1}, Y(2 S), \chi_{b 2}$ and $Y(3 S)$ ) mesons. Because of their large masses, charm and bottom pairs are produced only in the initial high energy parton collisions, with a small fraction of pairs forming quarkonium states. The time scale for development of these quarkonium states is such that they form while passing through the hot dense medium produced in a heavy ion collision. Thus the yield of the quarkonium states will be affected by any changes in their properties due to the final state medium. Because the quarkonium $\mathrm{S}$ states have a large decay branch to dileptons, they are easily observed and counted. But the production cross sections are low, so very large integrated luminosity is needed for high statistics measurements, particularly for excited charmonium and bottomonium states.

At sufficiently high energy density, color screening is expected to cause quarkonium to become unbound. This dissociation will occur at different temperatures for states of different size. Lattice gauge theory calculations (or, where they do not exist, potential models) suggest that the highest temperatures to which the charmonium states remain well defined (ie. bound) are $\sim 2 \mathrm{~T}_{\mathrm{c}}(\mathrm{J} / \psi), \sim$ $1.1 \mathrm{~T}_{\mathrm{c}}\left(\chi_{\mathrm{c}}\right)$, and $\sim 1.1 \mathrm{~T}_{\mathrm{c}}\left(\psi^{\prime}\right)$. For bottomonium, they are $\sim 4 \mathrm{~T}_{\mathrm{c}}(\mathrm{Y}(1 \mathrm{~S})), \sim 2 \mathrm{~T}_{\mathrm{c}}(\mathrm{Y}(2 \mathrm{~S}))$, and $\sim 1.1 \mathrm{~T}_{\mathrm{c}}$ $(\mathrm{Y}(3 \mathrm{~S}))$. Thus the quarkonium yields could act as a thermometer of the matter created in heavy ion collisions, given sufficiently accurate lattice calculations (although it should be emphasized that there are no definitive lattice calculations yet for many of these states). While lattice calculations can provide insight as to the highest temperature at which a state remains bound, models of iquarkonium production in heavy ion collisions must include the effects of the initial conditions and of the dynamical evolution of the system. Dynamical models include cold nucleus effects shadowing in the colliding nuclei leading to a reduction in the initial charm yields, and inhibition of quarkonia formation by collisions with nucleons in the colliding nuclei - as well as final state effects from the hot/dense matter. The time dependent conditions in the medium are taken from hydrodynamics calculations while the in-medium properties of the heavy quark states are from lattice calculations. Dissociation of quarkonia caused by collisions with high energy thermal gluons in the expanding medium leads to a reduction of the yield. But any bound quarkonium state can form in the medium by coalescence of uncorrelated charm or bottom pairs, if their density is large enough. To use quarkonia as a final state probe, cold nuclear matter effects must be unfolded from final state medium effects by separate measurements in $\mathrm{p}+\mathrm{p}$ and $\mathrm{p}+\mathrm{A}(\mathrm{or} \mathrm{d}+\mathrm{A})$ collisions at the same collision energy per nucleon. The ability to do this is a strong feature of the RHIC facility.

The RHIC quarkonium results available to date are PHENIX J/ $\psi$ measurements for $p p, d+A u$, $\mathrm{Cu}+\mathrm{Cu}$ and $\mathrm{Au}+\mathrm{Au}$ collisions. The results are from the central (mid-rapidity electron) and forward and backward (muon) arms. The $\mathrm{Au}+\mathrm{Au}$ and $\mathrm{Cu}+\mathrm{Cu}$ measurements include nuclear modification factors $\mathrm{R}_{\mathrm{AA}}$, preliminary rapidity distributions, and $\left\langle\mathrm{p}_{\mathrm{T}}{ }^{2}\right\rangle$ values - all as a function of collision centrality. The measured nuclear modification factors decrease smoothly with the number of participants to a factor of $\sim 3$ suppression for the most central $\mathrm{Au}+\mathrm{Au}$ collisions. So far, the preliminary rapidity distributions and $\left\langle\mathrm{p}_{\mathrm{T}}{ }^{2}\right\rangle$ data have substantial statistical and systematic errors.

Theoretical baseline calculations of cold nuclear matter effects that describe the $d+A u$ data can explain roughly half of the observed $\mathrm{J} / \psi$ suppression in heavy ion collisions. Most models containing cold nuclear matter effects plus suppression due to the presence of hot dense matter 
alone overpredict the observed suppression by a factor of three or more. Adding production of $\mathrm{J} / \psi$ by coalescence to the models increases the model yields to approximately the observed values. But since the $\mathrm{J} / \psi$ nuclear modification factor is predicted to be a balance between competing effects, this agreement cannot be decisive on its own. In fact, there are dissenting views on the importance of coalescence, so that independent experimental tests that are sensitive to the mechanism are required. In principle, the primordial and coalescence components will have different kinematic distributions since a generic feature of the coalescence process is that the regenerated $\mathrm{J} / \psi$ transverse momentum and rapidity distributions are narrower than those for the primordial $\mathrm{J} / \psi$. But, as noted earlier, the rapidity and $\left\langle\mathrm{p}_{\mathrm{T}}{ }^{2}\right\rangle$ data still have substantial uncertainties. In addition, theory predictions of the kinematic distributions are not yet very mature, leaving the issue unsettled.

More powerful and comprehensive tools for studying the effects of coalescence will require the luminosity upgrade. An important tool will be measurement of the $\mathrm{J} / \psi$ elliptic flow parameter $\mathrm{v}_{2}$, which has been very illuminating for hadrons containing lighter valence quarks. Also, the relative importance of $\mathrm{J} / \psi$ suppression and regeneration will change as a function of collision energy. The $\mathrm{J} / \psi$ yield in central collisions is predicted to be dominated by primordial production at SPS energy, and by regeneration at RHIC full energy. The luminosity upgrade will make it feasible to measure accurate $\mathrm{J} / \psi \mathrm{p}_{\mathrm{T}}$ and rapidity distributions for a range of collision energies. It should also make it feasible to measure $v_{2}$ in a long run at an intermediate collision energy, say at $62 \mathrm{GeV}$, where the primordial $\mathrm{J} / \psi$ yield is expected to dominate. The ability to make charmonium measurements at such energies will be unique to RHIC II.

Excited charmonium - the $\chi_{c}$ and $\psi^{\prime}$ states - cannot be studied at RHIC without the luminosity upgrade. Lattice calculations predict that these excited states should not exist in the QGP at RHIC. Therefore comparison of the $\chi_{c}$ and $\psi^{\prime}$ yields with the $\mathrm{J} / \psi$ yield as a function of centrality is considered to be a strong test for deconfinement.

The study of bottomonium states, the $\mathrm{Y}$ family, is also possible only at RHIC II luminosities. Bottomonium differs in two important ways from charmonium. First, the $\mathrm{Y}(1 \mathrm{~S})$ binding energy is higher so it should dissociate at higher temperatures. Second, there are only $\sim 0.05$ bottom pairs per central $\mathrm{Au}+\mathrm{Au}$ collision, making bottomonium production by coalescence much less likely. Thus bottomonium production at RHIC II will provide a very different window on color screening effects than charmonium production. The bottomonium yields at RHIC II should be sufficient for measurements of $\mathrm{R}_{\mathrm{AA}}$ as a function of centrality.

The larger cross sections at the LHC are approximately balanced by the increased luminosity and running times at RHIC II so that the heavy flavor yields per year are similar. Thus the types and quality of measurements that can be made at the two facilities will also be similar. However, there will be important differences in the physics environments at the two facilities that will make the programs nicely complementary.

The higher initial energy density at the LHC means that the QGP will be created at a significantly higher temperature. In addition, the factor of $\sim 10$ increase in charm pairs and the factor of $\sim 100$ increase in bottom pairs per central collision at the LHC will have a major impact on the interpretation of heavy flavor measurements. At the LHC, all of the charmonium states should be 
unbound at the highest temperatures. Thus the prompt charmonium yields at the LHC should be large and should reflect only coalescence, with no contribution from the primordial $\mathrm{J} / \psi$ production (except in very peripheral collisions). Thus measurements at RHIC and the LHC will resolve the ambiguities in interpretation of the data caused by the balance between destruction and coalescence formation of charmonium at RHIC.

Because of its higher binding energy, bottomonium at the LHC should behave similarly to charmonium at RHIC. The Y(1S) may remain bound at the highest temperatures at the LHC while the other bottomonium states will melt. Given $\sim 5$ bottom pairs per central $\mathrm{Pb}+\mathrm{Pb}$ collision (compared to $\sim 10$ charm pairs at RHIC), the Y yield at the LHC is predicted to reflect a balance between dissociation and coalescence reminiscent of the RHIC J/ $\psi$ production models. However, at RHIC the bottomonium dissociation rates will be significantly different. The Y(1S) is predicted to be bound, and the $\mathrm{Y}(2 \mathrm{~S})$ may be too. Only the $\mathrm{Y}(3 \mathrm{~S})$ is likely to melt at RHIC. Also, no significant bottomonium production by coalescence is expected. Thus the bottomonium yields at RHIC II should reflect only QGP suppression. Figure 11 shows predictions from one model for the relative suppression and recombination rates for Upsilons at RHIC and LHC. The measurements at RHIC II and the LHC will thus resolve the ambiguities in interpretation caused by the expected balance of bottomonium destruction and coalescence at the LHC.
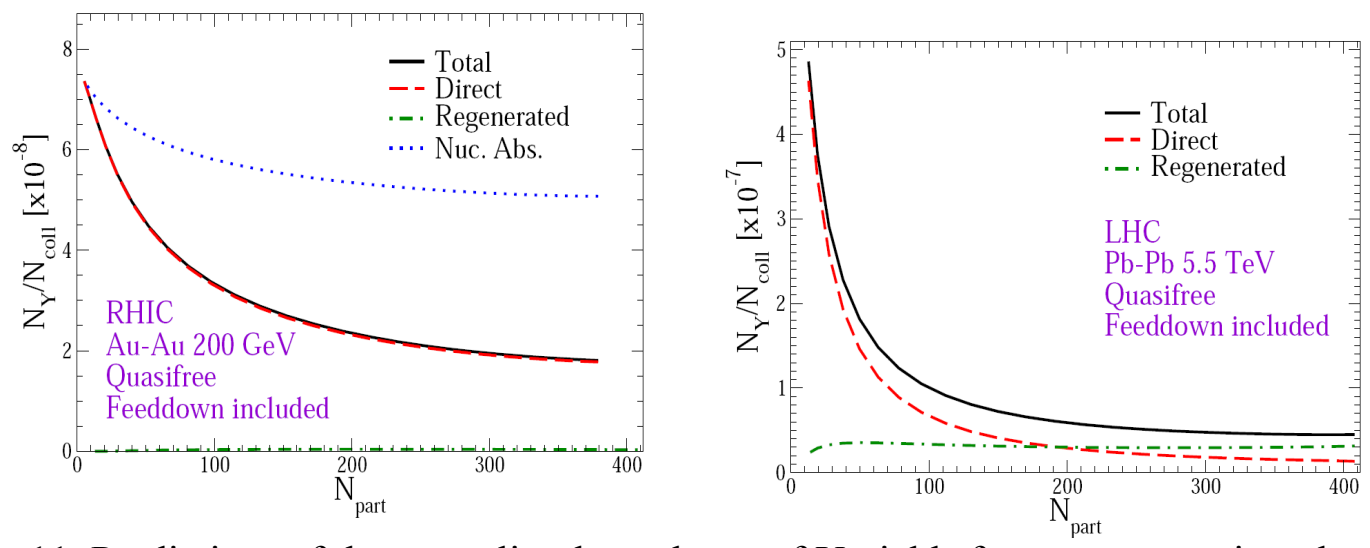

Fig. 11. Predictions of the centrality dependence of $Y$ yields from a suppression plus coalescence model at RHIC top energy (left) and at LHC top energy (right). The line labeled "Nucl. abs." in the left panel shows the estimated effect of cold nuclear matter processes only. 


\section{Charmonium and bottomonium at RHIC II and the LHC}

The lowest mass charm-anticharm (charmonium) and bottom-antibottom (bottomonium) mesons are bound so tightly that, according to lattice gauge results, they resist being destroyed by color screening until well above the phase transition temperature. The more weakly bound excited states are predicted to disappear at progressively lower temperatures. Along with their large decay branches into di-leptons, this makes the charmonium and bottomonium states powerful probes of the conditions in the QGP. For the charmonium states, the dissolution temperatures are estimated by lattice gauge or potential models to be $\sim 2 \mathrm{~T}_{\mathrm{c}}(\mathrm{J} / \psi), \sim 1.1 \mathrm{~T}_{\mathrm{c}}\left(\chi_{\mathrm{c}}\right)$, and $\sim 1.1 \mathrm{~T}_{\mathrm{c}}\left(\psi^{\prime}\right)$. For bottomonium, they are $\sim 4 \mathrm{~T}_{\mathrm{c}}(\mathrm{Y}(1 \mathrm{~S})), \sim 2 \mathrm{~T}_{\mathrm{c}}(\mathrm{Y}(2 \mathrm{~S}))$, and $\sim 1.1 \mathrm{~T}_{\mathrm{c}}(\mathrm{Y}(3 \mathrm{~S}))$. Here, $\mathrm{T}_{\mathrm{c}}$ is the critical temperature for the deconfinement phase transition.

A comprehensive program of measurements for charmonium and bottomonium states is planned at RHIC II and at the LHC. The higher production cross sections at the LHC are more than balanced by the higher luminosity and longer running times at RHIC II, and so the type and quality of the measurements will be similar at the two facilities. The figure shows the estimated annual recorded yield of several heavy flavor signals for PHENIX and STAR at RHIC II, and for ALICE and CMS at the LHC.

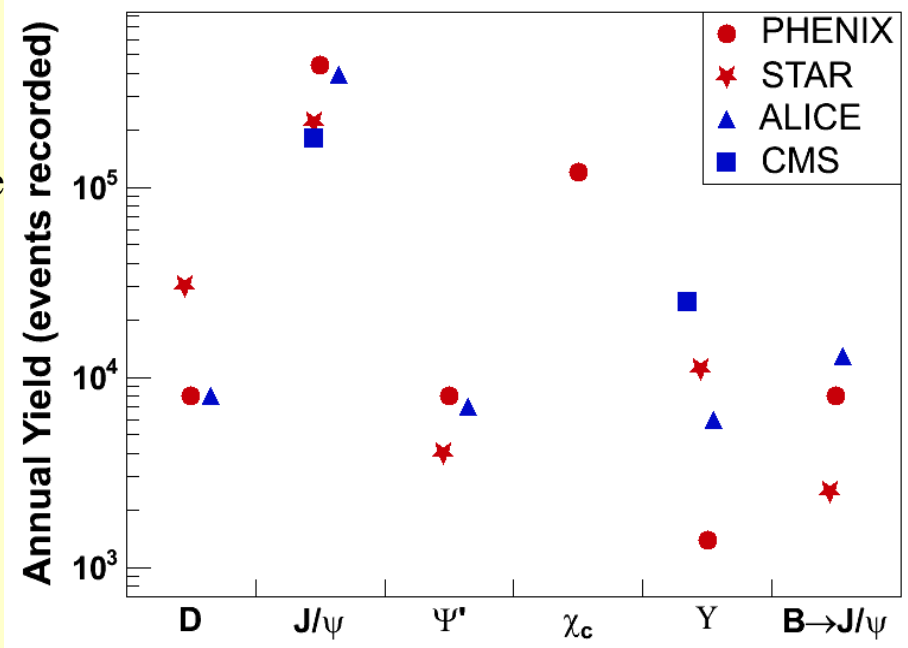

The physics environments, however, will be quite different at RHIC and LHC energies. The initial temperature will be higher at the LHC $\left(\sim 4 \mathrm{~T}_{\mathrm{c}}\right)$ than at RHIC $\left(\sim 2 \mathrm{~T}_{\mathrm{c}}\right)$, so that only the $\mathrm{Y}(1 \mathrm{~S})$ may remain bound at all temperatures at the LHC. The cross sections will be larger for charm pairs (x10) and bottom pairs (x100) at the LHC, and this will have a major impact on the interpretation of heavy flavor measurements. For example, $\mathrm{J} / \psi$ production at RHIC is expected to be due to both primordial production (in the initial nucleon-nucleon collisions) and coalescence of uncorrelated charm and anti-charm quark pairs in the QGP, while at the LHC it should be due entirely to coalescence. On the other hand, Y(1S) production at the LHC is expected to be due to a mix of primordial production and coalescence, while at RHIC only primordial production is expected to be important. Thus measurements at the LHC should help to resolve ambiguities due to competing mechanisms at RHIC, and vice versa, making the two programs highly complementary. 


\section{J/ $\psi$ elliptic flow measurements at RHIC II}

A diminished rate for $\mathrm{J} / \psi$ resonance production in $\mathrm{Au}-\mathrm{Au}$ collisions has long been predicted as a signature of deconfinement of quark-antiquark bound states in a quark gluon plasma. However, the number of observed $\mathrm{J} / \psi$ may also be influenced by the coalescence of uncorrelated pairs of charm quarks and antiquarks throughout the plasma volume. With the enhanced luminosity of RHIC II, these competing effects may be sorted out by measuring the $\mathrm{J} / \psi$ flow parameter (see sidebar, p. 16).

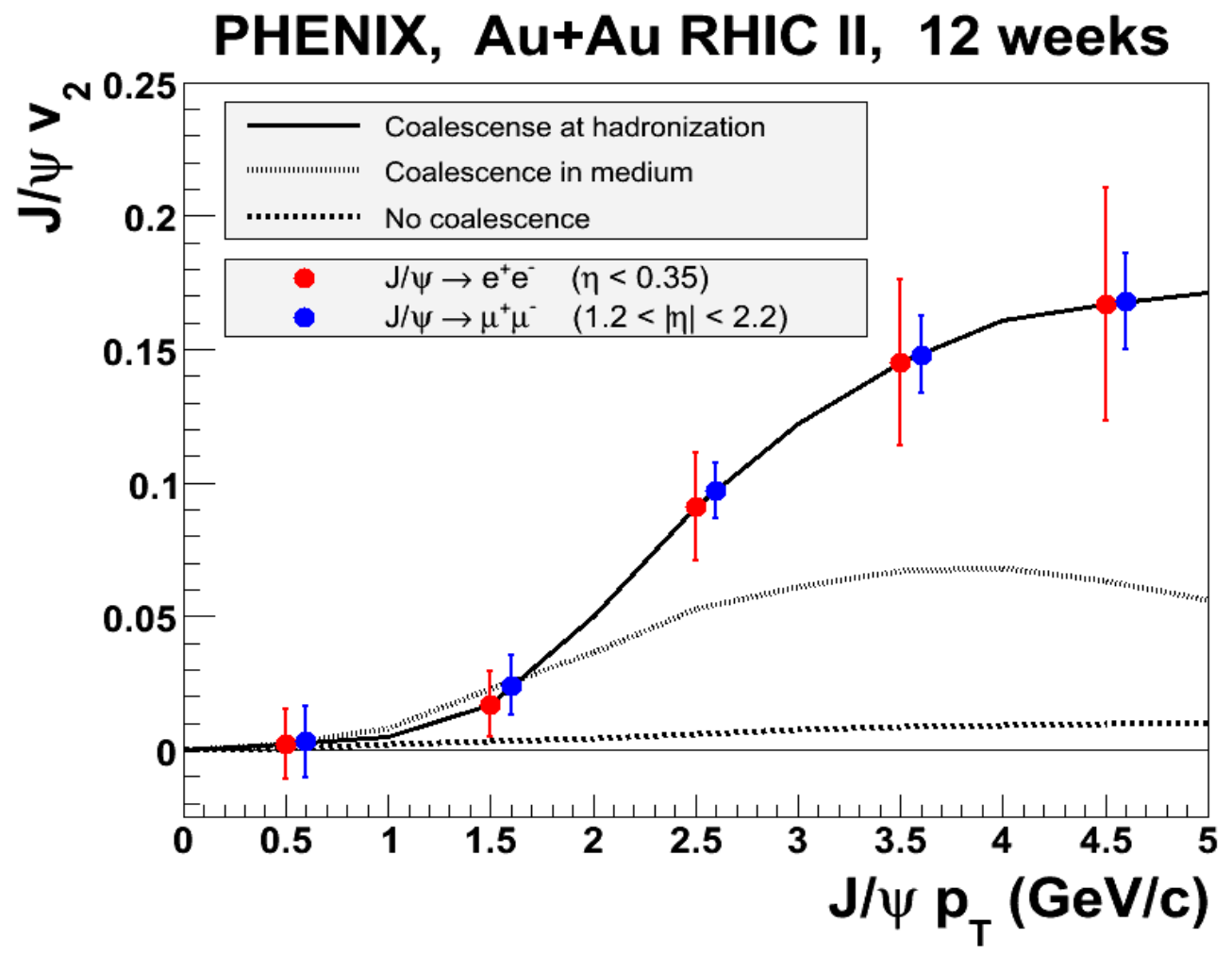

The figure shows the elliptic flow predicted for three extreme scenarios. The error bars denote the precision expected for measurements in the PHENIX detector for $\mathrm{J} / \psi$ decays to electron-pairs and muon-pairs for one year of $\mathrm{Au}+\mathrm{Au}$ running at RHIC II.

The largest elliptic flow is predicted if the $\mathrm{J} / \psi$ are formed from thermalized c quarks at the time when the plasma freezes out to form hadrons (see sidebar, p. 15). A smaller but still moderately large elliptic flow is predicted when the $\mathrm{J} / \psi$ form continuously in the plasma as the system expands, and a very small elliptic flow is predicted when there is no formation of $\mathrm{J} / \psi$ at all by coalescence. 


\subsubsection{Hadronization}

Hadronization is the process in which the partonic medium converts into hadrons. It includes both the dressing of quarks from their bare masses, thus breaking the approximate chiral symmetry, and the confinement of quarks into colorless hadrons. Due to its non-perturbative nature, the hadronization process is not understood at a fundamental level. Heavy ion reactions present an opportunity to study this process in the nucleus (using $\mathrm{d}+\mathrm{A}$ collisions) and in the dense QGP medium (A+A collisions). The detailed understanding of hadronization also plays a crucial role for isolating signatures sensitive to the evolution and properties of the QGP from those which are dominated by the later reaction stages. Conclusive experimental evidence for hadronization occurring from thermalized quark and gluon distributions can be interpreted as evidence for QGP formation.

The major RHIC discoveries related to hadronization are the enhanced baryon-to-meson ratios at intermediate $\mathrm{p}_{\mathrm{T}}$ that can not be attributed to parton fragmentation, the baryon/meson differences in the suppression patterns of hadron production (see Fig. 3) and the empirical scaling of elliptic flow strength with the number of quarks in the hadrons (see Fig. 4).

One of the theoretical achievements of the first several years of the RHIC program was the development of the recombination plus fragmentation model of hadronization. This is based on the observation that in an environment with a high density of partons recombination always dominates over fragmentation for an exponentially falling parton spectrum, but that fragmentation wins out eventually, when the spectrum takes the form of a power law. Thus hadron production in the intermediate $\mathrm{p}_{\mathrm{T}}$ region $(\sim 2-5 \mathrm{GeV} / \mathrm{c})$, is likely to be dominated by recombination. The success of recombination models in describing the observed baryon/meson differences gives strong evidence for the partonic nature of the medium formed at RHIC. Surprisingly, experimental studies of jet-correlations using identified baryon or meson triggers in the intermediate $\mathrm{p}_{\mathrm{T}}$ region show little or no particle species dependence, which rules out recombination from a pure thermal source. For the recombination picture to remain valid, at least one of the quarks in the baryon has to come from the fragmentation of a hard-scattered parton and thus preserve the jet-like correlations between the final hadrons.

A detailed study of the interplay of recombination and fragmentation will require high precision data on identified particle distributions covering $\mathrm{p}_{\mathrm{T}} \sim 8 \mathrm{GeV} / \mathrm{c}$. Of particular interest will be data on multi-strange hadrons and resonances. Resonances have been predicted to violate the elliptic flow scaling law, allowing for further insight into the strength of hadronic final state interactions. In addition, the measurement of dynamical two-particle correlations will help to constrain the recombination formalism and the transition to fragmentation at high $\mathrm{p}_{\mathrm{T}}$. Enhanced PID capabilities will become available after the planned STAR and PHENIX upgrades, while the RHIC II luminosity is needed to obtain statistically significant elliptic flow and correlation results involving rare particle species.

There have been attempts to describe the baryon/meson effects through the existence of gluon junctions. This approach poses a fundamental question about the nature of baryon number. Detailed baryon number versus charge transport measurements and the transverse momentum dependence of hadron chemistry are needed to enable the experimental test of whether gluons have 
a contribution to the baryon number. A full accounting for the baryon number transport requires the detection of neutrons and anti-neutrons, in addition to the already existing capabilities to detect charged and weakly decaying neutral hadrons. Such capabilities are not included in the present STAR or PHENIX upgrade programs. A spectroneter with a hadronic calorimeter combined with good particle identification for charged hadrons and good kinematic coverage would be needed to enable the measurements of baryon and charge transport over a broad kinematic range. Such a detector would presumably have small acceptance, and thus would require RHIC II luminosity for these measurements to be feasible. There are presently no plans for such a detector. 


\subsection{Gluon saturation}

Here we discuss the experimental program that will address the second of the fundamental questions, introduced in section 4.2, "what is the wave function of a nucleus?".

The gluon density in the nucleon has been measured at HERA over a very broad range in the momentum fraction $x$. At small $x$, parton splitting causes the gluon density to grow rapidly. This growth can not continue indefinitely due to unitarity limits. Thus, it has long been recognized that the gluon density in the nucleon must saturate at some scale, but the momentum fraction where this occurs is not yet experimentally well determined.

Heavy nuclei provide an ideal laboratory to explore gluon saturation. The gluon density per unit transverse area is amplified by a factor of $\sim \mathrm{A}^{1 / 3}$ relative to that in the nucleon, so saturation effects should become apparent at larger $x$ in nuclei than in the nucleon. This is best studied with $\mathrm{d}(\mathrm{p})+\mathrm{A}$ collisions at forward rapidity, where the low- $x$ gluons in the heavy nucleus are probed using the high- $x$ partons in the proton or deuteron. Data from all four RHIC experiments demonstrate that inclusive particle yields in $\mathrm{d}+\mathrm{Au}$ collisions are strongly suppressed at forward rapidity relative to those in $\mathrm{p}+\mathrm{p}$ collisions. The likely explanation for these results is that the onset of gluon saturation in the Au nucleus occurs in the region $x=0.001 \sim 0.01$ that is probed at RHIC. However, several other mechanisms have been proposed that are also consistent with the measurements to date. Thus, more detailed studies are required to elucidate the underlying dynamics. Multi-particle correlation measurements will be particularly informative. In conventional perturbative QCD, hard scattering processes are dominated by di-jets from $2 \rightarrow 2$ parton elastic scattering. In contrast, gluon saturation models predict that forward particle production will be dominated by multiple scattering of energetic partons off the gluon field of the nucleus, leading to the appearance of mono-jets. Detector upgrades to PHENIX and STAR will facilitate a search for mono-jets in $\mathrm{d}+\mathrm{Au}$ collisions and direct measurements of the gluon density in the Au nucleus within the next few years. Depending on what is learned, follow-up studies will include direct photon and di-lepton measurements. These rare probes, which will permit more precise, quantitative comparisons to theory, will require RHIC II luminosities.

It is essential for us to determine if gluon saturation is the correct explanation for the existing RHIC data. The saturation scale represents a fundamental property of hadrons and nuclei. Indeed, one saturation theory that has been quite successful, the Color Glass Condensate, predicts that there exists a universal wave function for hadronic matter at sufficiently high energy or small $x$ (see Fig. 12). Even if gluon saturation does not prove to be the correct explanation, it is nonetheless crucial to determine the gluon density at small $x$ in the Au nucleus if one hopes to understand the initial state in $\mathrm{Au}+\mathrm{Au}$ collisions at RHIC and its rapid evolution from a highly nonequilibrated system to a thermalized one.

RHIC is uniquely situated to explore this phenomenon. We know that the gluon density ultimately must saturate, but a fundamental question is: When does it occur? For example, the gluon density at the saturation momentum controls such basic properties of heavy-ion collisions as the multiplicity. To observe the onset of saturation, it's essential to be able to turn it on and off. This will likely be possible at RHIC. This would also make RHIC the ideal laboratory to explore the underlying mechanism for saturation. In contrast, particle production in heavy-ion collisions at the 
LHC may occur deep within the saturation regime over most of the accessible kinematic region. If that proves to be the case, then (a) it will be very difficult to turn saturation effects off at the LHC, and (b) it will be essential to quantify the saturation effects independently in order to distinguish between initial-state saturation effects vs. final-state plasma effects in $\mathrm{Pb}+\mathrm{Pb}$ collisions at the LHC.

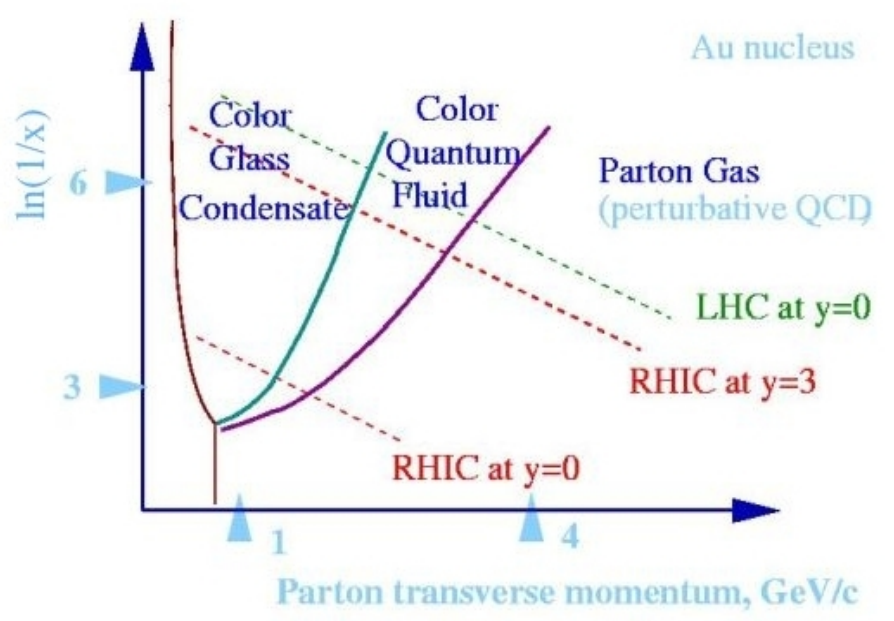

Fig. 12: A schematic picture of the high energy phase diagram of QCD within the Color Glass Condensate model. The vertical axis is a function of the parton momentum fraction, $\mathrm{x}$. 


\subsection{Spin structure of the nucleon}

The third fundamental question, introduced in section 4.3, "what is the wave function of the proton", is the motivation for the RHIC Spin program.

RHIC is the world's first - and only - polarized proton collider. Hence, RHIC has a unique role to play in the world-wide effort to elucidate the proton wave function through studies of its partonic spin structure. Deep-inelastic scattering of polarized leptons on polarized nucleon targets have shown that approximately $20-30 \%$ of the proton's spin can be attributed to the preferential alignment of the spins of the quarks and antiquarks. The rest of the proton's spin must arise from the preferential alignment of the gluon spins or from orbital motion of the quarks and gluons. The RHIC spin program goals include direct measurements of the gluon and flavor-separated quark and antiquark contributions to the proton's spin, and studies of the transverse spin and transverse motion preferences of the quarks and gluons in a transversely polarized proton.

Measurements are already underway at RHIC that are sensitive to the gluon helicity distribution $\Delta g(x)$ in a longitudinally polarized proton. First results are available from inclusive pion and jet production, and first measurements of direct photons and photon+jet coincidences will be performed over the next several years. The photon+jet channel is particularly important, as it provides direct access to the $x$-dependence of the gluon polarization. These efforts will be well advanced before RHIC II, but the subsequent increase in luminosity will make it practical to improve their precision and determine the integral gluon contribution to the proton spin to an uncertainty $(\sim \pm 0.1)$ comparable to the known contribution from quark and antiquark spin orientation. Around the end of this decade, a parallel effort will begin to exploit the flavordependence inherent in weak $\mathrm{W}^{ \pm}$boson production to determine the flavor-dependence of seaquark and antiquark polarizations in a polarized proton. But only with the integrated luminosities available from RHIC II will such studies reach their precision potential. Measurements of charmtagged $\mathrm{W}$ production to provide specific sensitivity to strange-quark polarization only become interesting with RHIC II luminosities. The same is true of transverse spin asymmetry measurements for Drell-Yan dilepton production, which will uniquely test our understanding of quark motional preferences (related to orbital angular momentum) transverse to the plane formed by proton spin and momentum. This suite of RHIC II spin measurements will significantly enhance our map of the proton's internal wave function in terms of its partonic constituents. At the same time, RHIC II will facilitate searches for possible physics beyond the Standard Model in spin observables for hard QCD processes.

The primary challenge that faces the RHIC spin program is to achieve the integrated luminosity necessary in order to satisfy its various complementary goals. The RHIC II luminosity upgrade will provide a factor $\sim 2-3$ improvement in the luminosity for pp collisions, which are not currently limited by intra-beam scattering. However, even this modest improvement, coupled with anticipated STAR and PHENIX detector upgrades, and possibly the addition of a relatively lowcost dedicated new detector, will extend the physics reach of the spin program in important ways.

Gluon Polarization: The key task for the first phase of the RHIC spin program is to explore the polarization of gluons, $\Delta g(x)=g^{+}(x)-g^{-}(x)$, in the proton. $\Delta g(x)$ counts the difference in numbers of gluons "spinning with" or "against" the proton, while carrying a fraction $x$ of the proton's momentum. Its measurement, therefore, sheds light on the question of how much of the proton spin 
is carried by gluons. This question has become a world-wide focus of the field ever since it was found in lepton scattering that the preferential orientation of quark and anti-quark spins carry unexpectedly little of the proton spin. A first determination of the gluon spin contribution is a DOE performance milestone for 2008, and initial information is now emerging from RHIC. At RHIC, a variety of probes of $\Delta g(x)$ are available, by observing photons, pions or QCD jets produced at large transverse momentum, or heavy quarks.

The cleanest probe for $\Delta g(x)$ - prompt-photon production - also happens to be the rarest. Integrated luminosities beyond those considered in the document "Research Plan for Spin Physics at RHIC" (submitted to DOE in February 2005) will clearly benefit the prompt-photon measurements, especially those involving correlations between a produced photon and jet. Such coincidence measurements can provide a direct determination of the $x$-dependence of the gluon polarization and important crosschecks on analysis assumptions (e.g., extracting information at the same $x$-values for quite different transverse momenta). In conjunction with planned detector upgrades, they will also extend the coverage in momentum fraction $x$. Depending on the shape of $\Delta g(x)$ to be found in the initial measurements at RHIC, such an extension may be crucial in order to determine the integrated gluon spin contribution to the proton spin with the desired precision: \pm 0.1 or $20 \%$ of the proton spin, the approximate size of the quark spin contribution.

Sea Quark and Antiquark Polarizations: Another focus at RHIC is the pattern of up, down, and strange quark and anti-quark polarizations in the nucleon's sea. Knowing these individually would likely give us much deeper insight into the question why the total proton spin contribution from quarks and anti-quarks is so small. For example, do the sea quarks and anti-quarks spin predominantly "against" the proton, thereby counteracting the contribution of valence quarks? The flavor-dependence of these parton polarizations is furthermore an important test for models and lattice-QCD predictions of nucleon spin structure and the origin of the sea from the distinct mechanisms of gluon splitting and virtual meson emission and reabsorption. For example, many models predict a basic sign difference between the polarizations of up vs. down anti-quarks. Definitive information addressing this prediction will begin emerging from RHIC several years from now, via studies of parity-violating single-spin asymmetries in $\mathrm{W}^{ \pm}$production. $\mathrm{W}^{+}\left(\mathrm{W}^{-}\right)$ bosons couple to left-handed up (down) quarks and right-handed down (up) anti-quarks and hence are natural, clean probes of the helicity and flavor structure in the proton's sea. A sufficient yield of W's in pp collisions at RHIC is only attainable with high-luminosity running at $\sqrt{s}_{s}=500 \mathrm{GeV}$. Upgrades of the STAR (forward tracking) and PHENIX (forward triggering) detectors are vital as well. The extractions of quark and anti-quark polarizations from $\mathrm{W}$ measurements constitute another DOE perfomance milestone, with initial results anticipated by 2013.

Integrated luminosities in excess of $1 \mathrm{fb}^{-1}$, as targeted for RHIC-II, would turn the inclusive W production measurements into high-precision studies of polarized quark and anti-quark densities, as illustrated in Fig. 13. Furthermore, the increased luminosity will make it feasible to extract new direct information on the polarization of strange $[\Delta s(x)]$ or anti-strange $[\Delta($ anti-s) $(\mathrm{x})]$ quarks in the proton. Many researchers in the field believe that these quarks play a critical role in accounting for the small observed net quark spin contribution to the proton spin. RHIC-II sensitivity to strange quarks would be provided by spin asymmetries in "charm-tagged W production": $p \mathrm{p} \rightarrow \mathrm{W}+($ anti- $)$ $\mathrm{c}+\mathrm{X}$. The underlying parton processes involve interaction of a strange (for $\mathrm{W}^{-}$) or anti-strange (for $\mathrm{W}^{+}$) quark from one proton with a gluon from the other, via one strong and one weak vertex. Competing sensitivities to gluon and to strange quark polarization could be unraveled by 
measurement in different kinematic regions, as illustrated in Fig. 14. Alternative sensitivity to $\Delta s(x)$ and $[\Delta($ anti-s $)(\mathrm{x})]$ could be provided by measuring the transfer of longitudinal polarization from colliding protons to $\Lambda$ and anti- $\Lambda$ baryons produced at high transverse momentum. The $\Lambda$ and anti- $\Lambda$ polarizations reflect primarily the spin orientation of their valence strange and antistrange quarks, respectively.
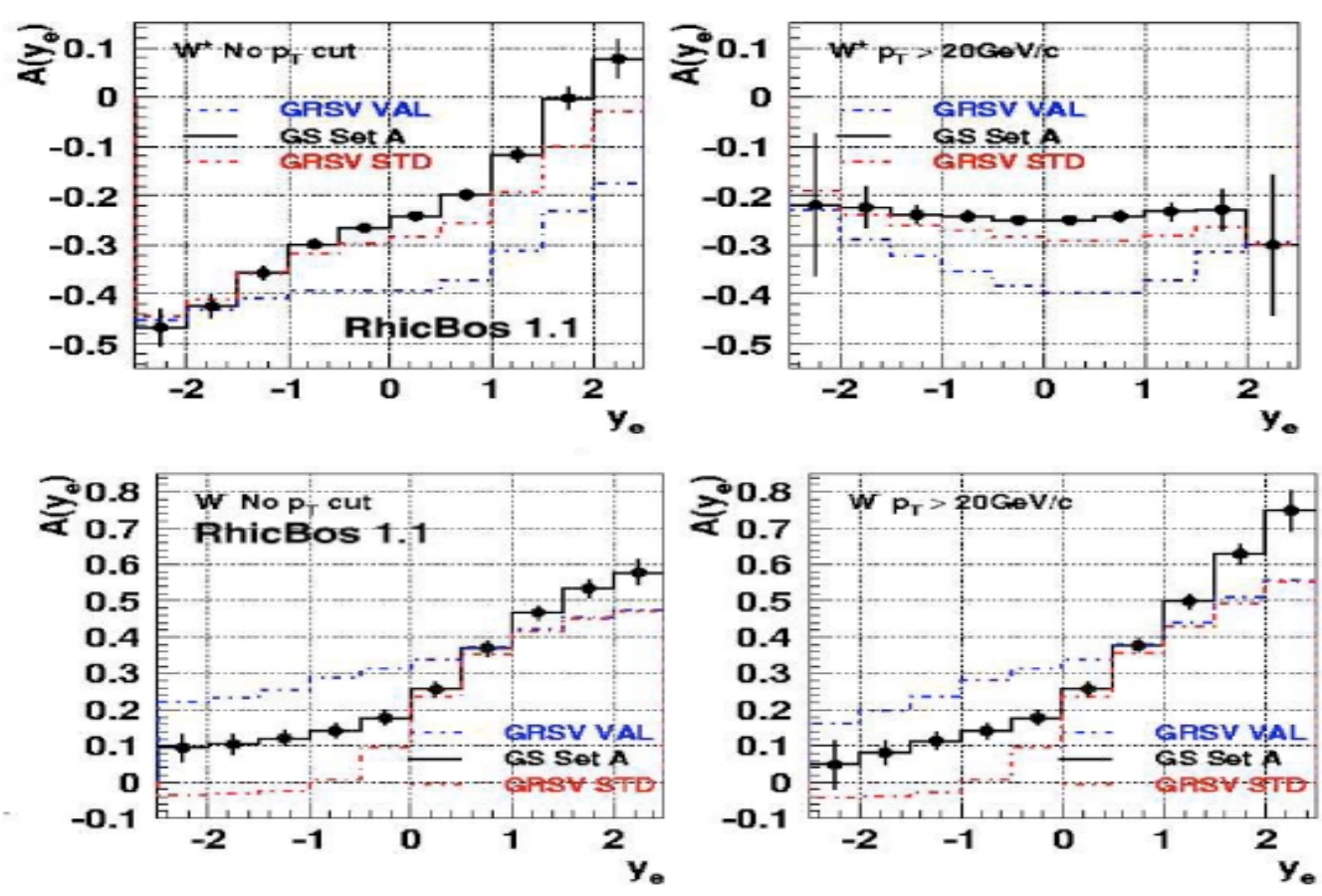

Figure 13. Single-spin parity-violating longitudinal asymmetry projections for inclusive $\mathrm{W}^{+}$and $\mathrm{W}^{-}$production attainable at RHIC-II with $800 \mathrm{pb}^{-1}$ of recorded luminosity and $70 \%$ beam polarization at $500 \mathrm{GeV}$ center-of-mass energy. Projected statistical uncertainties are plotted as a function of the rapidity of the detected daughter electron or positron, and are compared to predictions utilizing three different parton distribution sets to illustrate the sensitivity of the measurements.

Transverse Spin Measurements: The sizable single transverse spin asymmetries already observed in pp collisions at RHIC may arise from both transverse spin preferences ("transversity") and transverse motion preferences ("Sivers functions") of quarks and gluons in a proton polarized transversely to its momentum. Measurements intended to unravel these competing contributions, each of considerable interest in its own right, will be initiated at RHIC in the coming years, but may only come to full fruition with RHIC-II. An experimental map of transversity would yield the nucleon's tensor charge, one of its fundamental charges, and would complete the set of so-called "leading twist" parton distributions in the proton, which depend only on longitudinal momentum fraction $x$ and momentum transfer $Q^{2}$. Differences between the helicity and transversity densities give information about relativistic effects in the nucleon. Sivers functions describe correlations among proton spin and momentum and parton transverse momenta within the proton, and provide 
information related to parton orbital angular momenta.

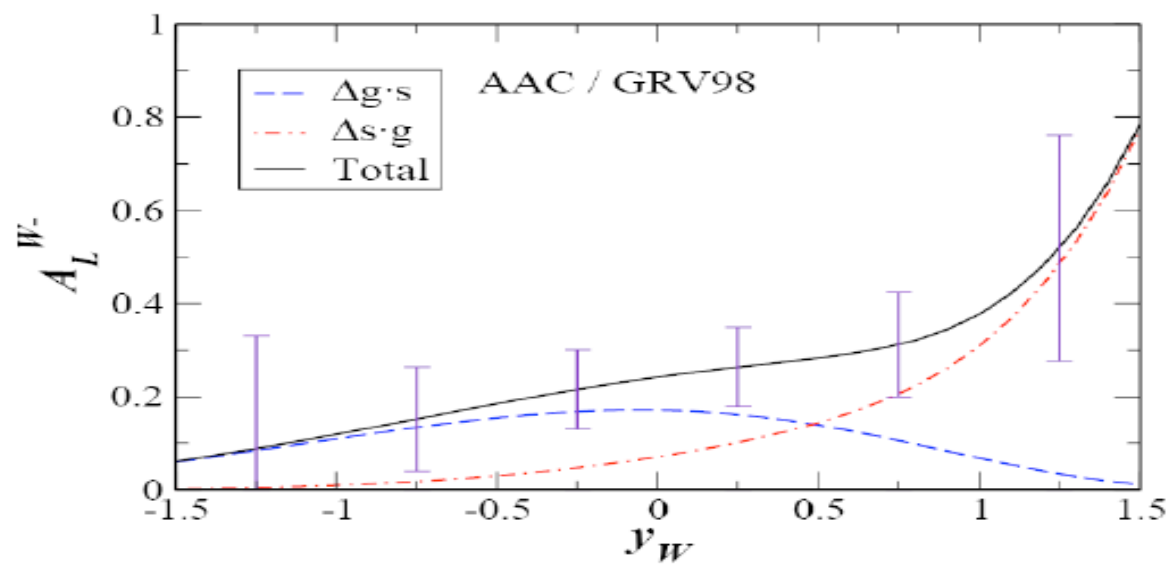

Figure 14. Leading-order contributions from gluon $(\Delta \mathrm{g})$ and strange-quark $(\Delta \mathrm{s})$ polarization to the parity-violating asymmetry for charm-tagged $\mathrm{W}^{-}$production, compared to statistical uncertainties achievable for $800 \mathrm{pb}^{-1}, 70 \%$ beam polarization, and 10\% detection efficiency for the electroncharm coincidence. The strange quark polarization dominates the asymmetry at positive rapidity of the $\mathrm{W}^{-}$, which leads to forward-going high- $\mathrm{p}_{\mathrm{T}}$ daughter electrons.
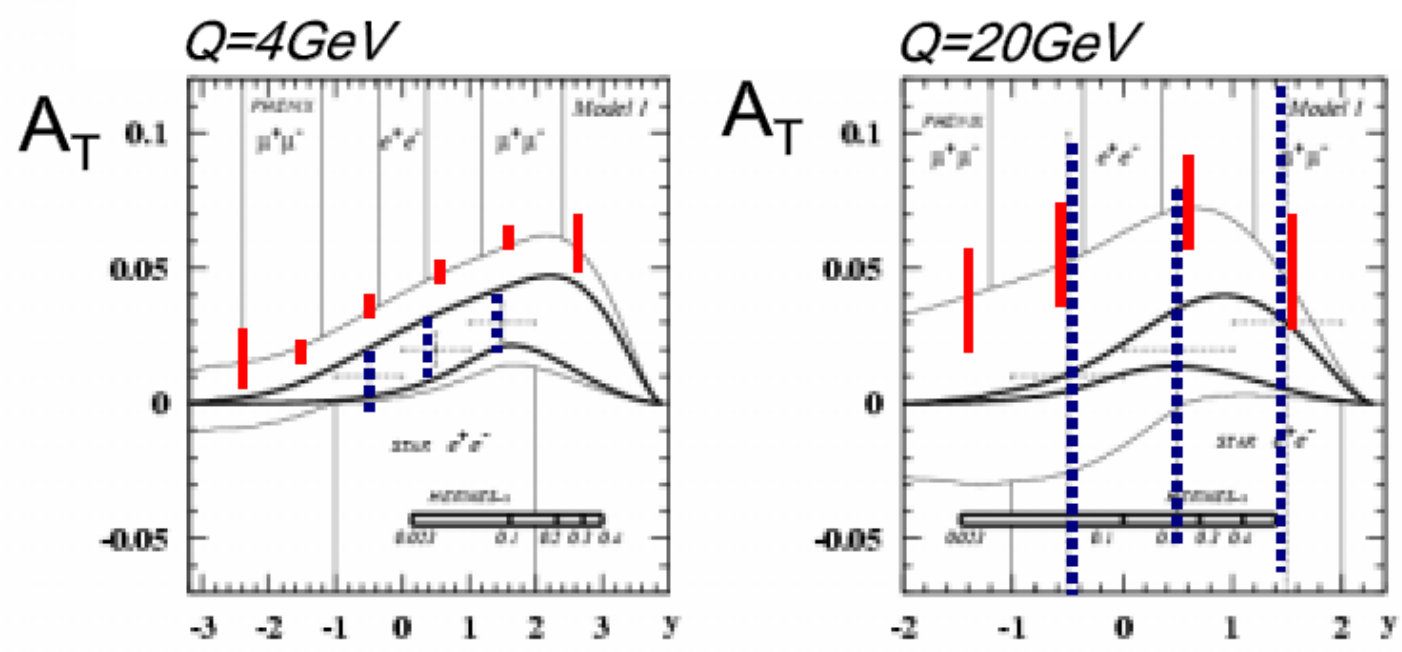

Figure 15. Single Spin asymmetries $A_{T}$ for Drell-Yan di-lepton production at RHIC II; projected errors are compared to theoretical projections by Menzel et al. in two invariant mass bins: $Q=4$ $\mathrm{GeV}$ (left) and $\mathrm{Q}=20 \mathrm{GeV}$ (right). The dashed lines show the errors expected from a measurement with the STAR detector, $\int \mathrm{Ldt}=125 \mathrm{pb}^{-1}$. The solid error bars correspond to a measurement with a future large acceptance experiment dedicated to Drell-Yan physics with $\int \mathrm{Ldt}=1250 \mathrm{pb}^{-1}$. It is anticipated that the new detector will take data with transverse spin continuously (factor 3-4 improvement over STAR and PHENIX) and that it will take advantage of special vertex focusing magnets to enhance the luminosity by an additional factor 3-5. 
The Sivers functions are associated with a rescattering of the struck parton in the color field of the nucleon remnant, a final-state interaction predicted to be attractive in the case of deep inelastic lepton scattering (such as studied in the HERMES and COMPASS experiments at DESY and CERN) but repulsive in Drell-Yan dilepton production. Therefore, the Sivers functions contribute with opposite signs to the single-spin asymmetries for these two processes. At RHIC-II luminosities, with a low-cost new detector dedicated to this purpose, it will be possible to measure transverse spin asymmetries for Drell-Yan processes with sufficient precision to verify this fundamental QCD prediction, which really tests all concepts we know of for analyzing hard hadronic processes. As a possible Drell-Yan detector we have studied a calorimeter experiment with large acceptance, $|\eta|<3$. The detector could be located at an interaction point without spin rotator magnets (that is $100 \%$ transverse spin) and will take advantage of an additional factor 3-5 in luminosity from new vertex focusing magnets. Projected error bars for RHIC II measurements of single spin asymmetries measurements in Drell-Yan are shown in Fig. 15.

Simultaneous measurement of the Drell-Yan sensitivity to the transverse spin orientations of both proton beams could allow an unraveling of transversity for anti-quarks vs quarks in the proton. 


\subsection{Non-equilibrium processes}

The fourth fundamental question, introduced in section 4.4, "what is the nature of non-equilibrium processes in a fundamental theory?" motivates the measurements discussed in this section.

The problem of CP invariance in strong interactions is of fundamental importance. It has farreaching implications for the cosmology of the Early Universe (for example, CP-odd effects at the deconfinement phase transition could help to understand the origin of the strong magnetic fields in space). The breaking of $\mathrm{CP}$ at the QCD phase transition and the associated generation of ChernSimons number is analogous to the generation of baryon number at the electro-weak phase transition. It is clear that experimental studies of $\mathrm{CP}$ violation at RHIC are important and would enrich our understanding of bulk non-equilibrium dynamics in QCD and related gauge theories.

The strong CP problem remains one of the most outstanding puzzles of the Standard Model. Even though several possible solutions have been put forward (for example, the axion scenario), at present it is still not clear why $\mathrm{P}$ and $\mathrm{CP}$ invariances are respected by strong interactions in spite of the possible presence of a CP-odd " $\theta$-term" in the QCD lagrangian ${ }^{1}$. Moreover, it was proposed that in the vicinity of the deconfinement phase transition the QCD vacuum can contain metastable domains possessing non-trivial topological Chern-Simons number and inducing $\mathrm{P}$ and $\mathrm{CP}$ violation. It was also suggested that this phenomenon would manifest itself in specific correlations of pion momenta. Such "P-odd bubbles" are a particular realization of an excited vacuum domain which may be produced in heavy ion collisions. Several dynamical scenarios for the decay of Podd bubbles have been considered, and numerical lattice calculations of the fluctuations of ChernSimons number in classical Yang-Mills fields have been performed.

The studies of P- and CP-odd correlations of pion momenta have shown that such measurements are feasible with large event samples. This also applies to the interesting idea of using $\Lambda$-hyperon spin correlations. Recently, it has been proposed that P- and CP-breaking in a domain of a highly excited vacuum state can manifest itself in heavy ion collisions through the charge asymmetry of the produced hadrons with respect to the reaction plane. In other words, a CP-odd domain would induce an electric dipole moment of the quark-gluon plasma, much like a non-zero $\theta$-term induces an electric dipole moment of the neutron. It should be noted that the most stringent limits on CPodd effects in "cold" QCD vacuum result from the neutron electric dipole moment measurements. The estimated magnitude of the expected charge asymmetry $(\sim 1 \%)$ makes the effect amenable to observation in existing and/or future heavy ion experiments. A method of measuring the charge asymmetry based on the "mixed harmonics" technique has been proposed. Very recently, the STAR Collaboration performed a preliminary analysis of the charge asymmetry in $\mathrm{Au}+\mathrm{Au}$ collisions at $\sqrt{\mathrm{s}}_{\mathrm{s}} \mathrm{A}=62 \mathrm{GeV}$. The results look promising, even though the systematic uncertainties have still to be carefully analyzed. All of the experimental studies described above will benefit from large event samples and from detector upgrades.

The mechanisms of entropy production, rapid thermalization and chemical equilibration in heavy

1 At zero temperature and density, a theorem by Vafa and Witten states that $\mathrm{P}$ and CP cannot be broken in the true ground state of QCD. However, this theorem does not apply to QCD matter at finite temperature and density. 
ion collisions at RHIC that lead from the initial state to thermalized matter are not yet understood, and the measurements needed to guide theoretical efforts are among the more difficult of the future RHIC measurements.

As discussed in section 5.1.1, comparison of data from RHIC with hydrodynamic models indicates strongly that thermalization of the new form of matter is very fast $(<1 \mathrm{fm} / \mathrm{c})$. The experimental program that is needed to understand the rapid thermalization mechanisms has strong overlaps with that needed to understand the properties of the thermalized medium. The thermalization process must be modeled using a microscopic description of the pre-equilibrium system dynamics, and the microscopic transport models need experimental input to constrain the transport coefficients in the medium. Heavy quarks are a good probe of the transport properties of the medium because, due to the large c-quark mass, the charm equilibration time is expected to be about a factor of 5 larger than the the light quark equilibration time. Theoretically, the charm diffusion coefficient has been related to the charm energy loss and momentum broadening. Utilizing transport theory, these quantities can then be used as an input for the calculation of charm elliptic flow, thus creating a relation between an experimentally accessible quantity and the diffusion coefficient.

Measurements of open charm production and flow data in heavy ion collisions are challenging due to the small production cross-sections and the experimental limitations on charm particle identification. While the $\mathrm{R}_{\mathrm{AA}}$ measurement from charm semileptonic decay data already has discriminative power, the statistical errors on the $\mathrm{v}_{2}$ results will have to be greatly improved to allow definitive tests of models. This will be achieved by a combination of detector upgrades and the RHIC II luminosity upgrade. Specifically, the vertex detector upgrades in PHENIX and STAR will make possible direct charm and beauty identification over a broad kinematic range with reduced backgrounds, and reaction plane resolution will improve by as much as a factor of two due to improved kinematic coverage.

As discussed in section 5.1.2.2, where the focus was on the properties of the thermalized medium, the composition of the pre-equilibrium and just thermalized matter and the chemical (ie. quark and gluon) equilibration mechanisms are also open questions. From the prevalence of gluons in the relevant $\mathrm{x}$-range of the incoming nuclei one might expect the early matter to be a gluon plasma (GP). However, recent calculations of quark-antiquark pair production within the classical fields generated by the incoming Au nuclei indicate a rather fast approach to chemical equilibrium. A way to test these questions is by dileptons, which maximally distinguish between gluons and quarks (with zero, $1 / 3$ or $2 / 3$ electric charges, respectively). The challenge will be to disentangle the contributions to the dilepton spectrum from thermal dileptons and non-thermal sources including Drell-Yan, correlated open-charm decays, the pre-equilibrium contribution, and jetplasma interactions. The goal will be to understand the composition of the early matter in terms of quark and gluon degrees of freedom. 


\section{RHIC II and other future heavy ion facilities}

RHIC II is expected to study heavy ion collisions and pursue the RHIC Spin program for a total of approximately 30 weeks per year. The initial temperature is $\sim 2 \mathrm{~T}_{\mathrm{C}}$ at RHIC maximum collision energy for $\mathrm{Au}+\mathrm{Au}$. RHIC can collide any combination of beams from $\mathrm{p}$ to $\mathrm{U}$ at the same energy, with rapid changes between species that enable the study of multiple species in one year. For heavy ions the collision energy can be varied from $\sqrt{\mathrm{s}}=5-200 \mathrm{GeV}$ and the baryo-chemical potential from $30-550 \mathrm{MeV}$ at central rapidity, allowing exploration of a large part of the QCD phase diagram. In addition, RHIC has the unique capability of being able to collide polarized protons at up to $\sqrt{\mathrm{s}}=500 \mathrm{GeV}$, in an energy region where pQCD can be used to describe the hard collision processes.

Two other heavy ion facilities are planned to operate in a similar time frame to RHIC II. These are the Large Hadron Collider (LHC) at CERN, which will devote 1 month per year to very high energy heavy ion collisions, and the Future Facility for Anti-proton and Ion Research (FAIR) at GSI, which will have a lower energy, fixed target facility for heavy ion collisions. In this section we will try to show how these facilities enrich the relativistic heavy ion field and complement the RHIC II program.

The LHC heavy ion program should start in 2009, and will receive 1 month of beam time per year. It will study $\sqrt{\mathrm{s}}=5500 \mathrm{GeV} / \mathrm{A}$ heavy ion collisions and $8800 \mathrm{GeV} / \mathrm{A} \mathrm{pA}$ collisions using a dedicated heavy ion detector (ALICE) and two detectors (ATLAS and CMS) that were designed primarily for pp collisions, but will work very well for some key heavy ion measurements. The pp reference data will be obtained, at least initially, using interpolated results from Fermilab and the $\sqrt{s}_{\mathrm{s}}=15000 \mathrm{GeV}$ pp running of the high energy physics program. Central $\mathrm{Pb}+\mathrm{Pb}$ collisions at the LHC are expected to produce initial temperatures of roughly $4 \mathrm{~T}_{\mathrm{C}}$, or about twice the estimated initial temperature at RHIC, at very low baryo-chemical potential. Jet cross sections will be much larger, and the transverse momentum reach of jets will be far larger than that at RHIC. Because of the very high collision energies, initial state gluon saturation effects, extremely interesting in their own right, are expected to be strong. Heavy flavor cross sections, particularly for bottom and bottomonium, are expected to be much larger than at RHIC II. This will roughly compensate for the shorter running time and lower luminosity of LHC.

The FAIR facility at GSI is planned to be completed in 2014. It will, among other things, provide up to $35 \mathrm{GeV} / \mathrm{A} \mathrm{U}$ beams for fixed target experiments using the CBM (Compressed Baryonic Matter) detector. For a $\mathrm{U}$ target, this is $\sqrt{\mathrm{s}}_{\mathrm{s}} 8 \mathrm{GeV}$. It will study the QCD phase diagram in the baryon rich region - where matter is at large baryo-chemical potential $(400-800 \mathrm{MeV})$ and moderate temperature. The major physics goals include studying in-medium properties of hadrons to look for evidence of the onset of chiral symmetry restoration, studying strangeness in dense baryonic matter, looking for evidence of deconfinement at high baryon densities, and searching for the QCD critical point using event-by-event fluctuations. As a fixed target facility, FAIR will provide much higher luminosities. As a collider facility, RHIC will provide detector systematics that are relatively independent of energy.

Figure 16 is an attempt to show schematically the relationship between the three future facilities by showing the regions of the QCD phase diagram accessed by each one. 


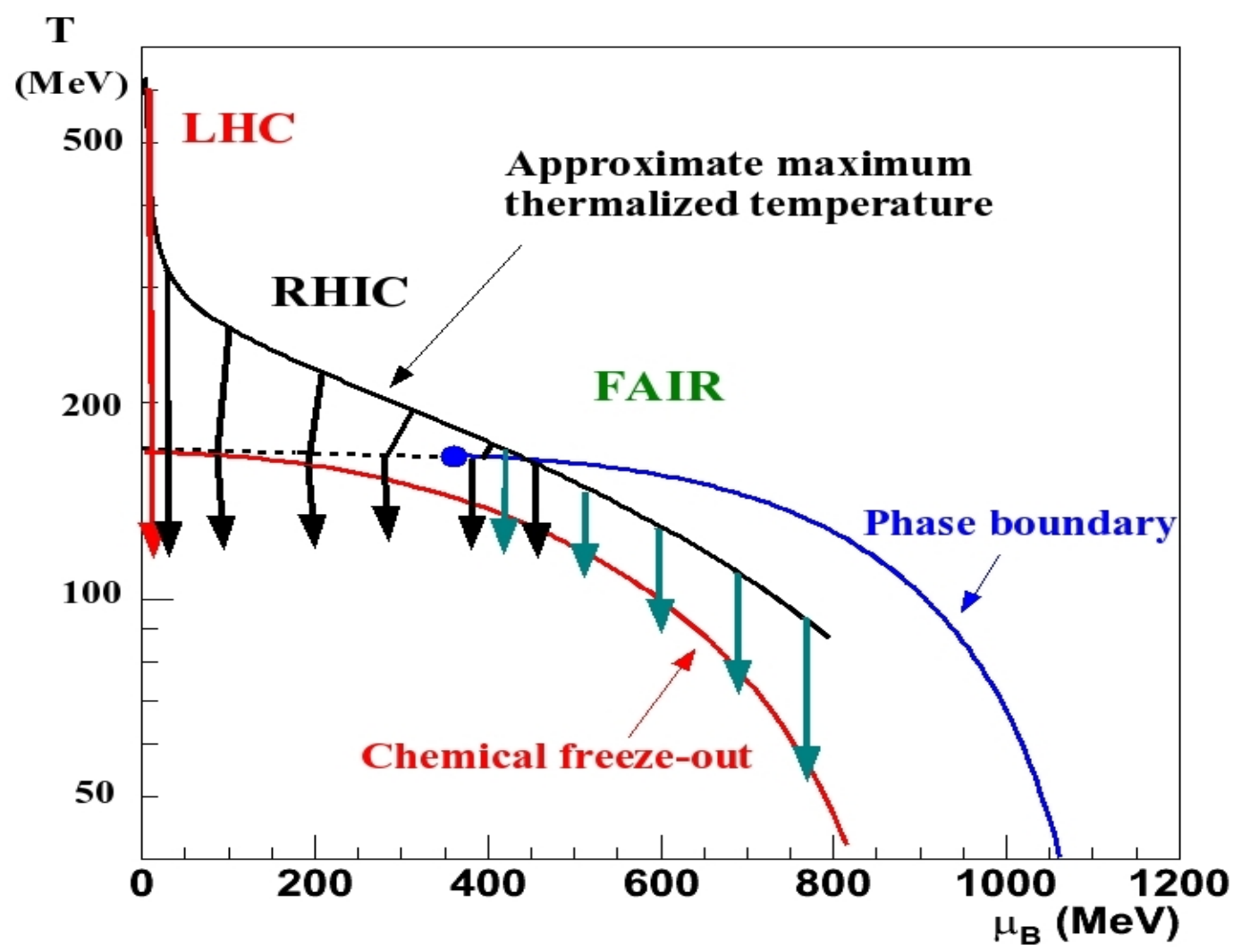

Figure 16. Schematic representation of the areas of the QCD phase diagram accessible at midrapidity to RHIC II at BNL, the LHC at CERN and FAIR at GSI. The location of the critical point, shown here as the point on the phase boundary curve between the line of first order phase transitions (solid) and the rapid crossover region (dashed), is presently unknown.

A brief summary of ways in which RHIC II, LHC and FAIR are complementary follows:

- The maximum initial temperatures at RHIC and LHC are different by a factor of $\sim 2$. This may result in weaker coupling strengths during the early development of the final state at LHC, and will certainly lead to a longer time ( $\sim$ twice as long) spent above the transition temperature. The two facilities together will place much stronger constraints on models of the final state medium than either one could alone.

- The heavy flavor programs at RHIC II and LHC will be strongly complementary. It will be possible to study charmonium and bottomonium production at initial temperatures well above the transition temperature, but under such different conditions of temperature and heavy quark multiplicity that different production mechanisms will dominate at the two facilities. Open heavy flavor energy loss can be studied at very different transverse momenta, very different energy densities of the medium, and possibly with significantly 
different energy loss mechanisms. Annual charm and bottom yields will be similar at RHIC and LHC because the higher luminosity at RHICoffsets the lower cross sections.

- The transverse momentum reach of jets at the LHC will extend far beyond that at RHIC, so that studies of the medium interactions of much higher energy jets will be possible at the LHC. Because of the much smaller background from multiple jets, RHIC will be very well suited to studies of lower momentum jets (which lose a large fraction of their energy) and of jet-medium recombination effects at intermediate $\mathrm{p}_{\mathrm{T}}$.

- While the LHC pushes to higher temperatures and lower baryo-chemical potential, RHIC II will have the ability to explore a large range of baryo-chemical potential, from 30 to 500 $\mathrm{MeV}$, by varying the collision energy from $\sqrt{\mathrm{s}}=5-200 \mathrm{GeV} / \mathrm{A}$. The ability to quickly change beam combinations and collision energies, combined with increased luminosity, will enable RHIC II to study a large portion of the QCD phase diagram. Of particular interest will be a search for the QCD critical point.

- RHIC II and FAIR will have different, slightly overlapping coverage in baryo-chemical potential. Together, they will be able to search for the QCD critical point over the baryochemical potential range from $30-800 \mathrm{MeV}$.

- RHIC II and FAIR will have complementary programs studying in-medium properties of hadrons via low mass dilepton pairs, with lower baryo-chemical potential at RHIC II and higher baryo-chemical potential at FAIR, while low mass dilepton pair measurements at the LHC would be very difficult because of the large background from open charm. This is a very important program that will, hopefully, provide information about the onset of chiral symmetry restoration in the region of the phase transition. 


\section{Relationship to other areas}

The physics of heavy ions and spin over the next decade at RHIC and RHIC II and new insights into some of our most fundamental questions are what drive the program. In addition, relationships to other areas of physics are providing new connections and ideas and expanding the scope and breadth of the field. Below we highlight some of these new connections.

6.1. Plasma physics: The evidence for formation of a strongly-coupled quark-gluon plasma at RHIC has generated great interest among physicists studying matter at high energy density. High energy density physics is a rapidly growing field that spans a wide range of physics areas including plasma physics, laser and particle beam physics, nuclear physics, astrophysics, atomic and molecular physics, materials science and condensed matter physics, intense radiation-matter interaction physics, fluid dynamics, and magnetohydrodynamics. These research areas all have common physics issues and intellectual challenges characteristic of matter under high energy density conditions. For example, understanding the fundamental properties of warm dense matter is an important thrust in plasma physics. Warm dense matter is an extreme state that is neither 'cold, condensed-matter', nor a 'hot, plasma' state, but rather somewhere intermediate. It is typically a strongly-coupled, many-body charged particle system with energy density in the $10^{11}$ $\mathrm{J} / \mathrm{m}^{3}$ range, conditions that are extremely difficult to study analytically and by numerical simulation. Many astrophysical systems (e.g., brown dwarfs, and giant planets) and laboratory experimental conditions fall into this regime. Dusty plasmas are strongly coupled and their properties and phase structure are being investigated by plasma and condensed matter physicists. Theoretical and experimental studies of the properties of the strongly coupled quark-gluon plasma address a system with similar underlying physics and even greater complexity due to the nonabelian nature of the interaction. There is, consequently, a unique opportunity for interdisciplinary collaboration. The field theory techniques required for non-perturbative QCD, along with experimental techniques such as elliptic flow measurements to study the time-integrated quark gluon plasma observables, may be applicabe to other high energy density systems.

6.2. Condensed matter physics: Recent advances in high-energy nuclear physics at RHIC and in condensed matter physics have focused attention on strongly correlated systems (systems in which the interaction energy of the constituents is large compared to their kinetic energy). At RHIC, the experiments suggest the existence of a strongly coupled quark-gluon plasma with unexpected properties. The quantitative theory of this novel phase of matter is still lacking, and to develop it would require invoking all of the expertise accumulated to date in the studies of strongly correlated condensed matter systems. Solving this complex and exciting problem requires a genuine interdisciplinary effort, involving professional researchers from both high energy nuclear physics and condensed matter physics. For example, one surprising property of the new matter discovered at RHIC is an extremely low viscosity, which makes it an almost perfect fluid. Fluids with small viscosity and "superfluids" have been a traditional area of research in condensed matter physics, and a detailed quantitative understanding of their properties has been achieved. Cold systems of atoms confined in magnetic - optical traps allow the study of the dependence of collective hydrodynamic behavior and viscosity on the coupling strength, which can be tuned at will via Feshbach resonances. This may help our understanding of the mechanisms responsible for the low viscosity of the QGP. On the other hand, the rich structure of QCD as a non-Abelian gauge theory allows for novel effects and phenomena which may still be unknown, so a study of quark-gluon 
matter may also stimulate new directions in condensed matter physics..

6.3 Cosmology: RHIC collisions create the energy density which characterized the Universe in the first few micro-seconds of its existence. Detailed information about the properties of the quarkgluon plasma and the dynamics of the quark-hadron phase transition will therefore advance our understanding of the cosmology of Early Universe. In particular, the fluctuations of the baryon number at the phase transition affect the spatial distribution of baryons and thus determine the initial conditions for nucleo-synthesis. Fluctuations of chromo-magnetic fields in the plasma may, through the coupling to charged quarks, have an effect on the "primordial" magnetic field of the Universe.

As discussed in more detail in section 5.4, the problem of CP invariance in strong interactions is of fundamental importance. It has far-reaching implications for the cosmology of the Early Universe (for example, CP-odd effects at the deconfinement phase transition could help to understand the origin of the strong magnetic fields in space). The breaking of CP at the QCD phase transition and the associated generation of Chern-Simons number is analogous to the generation of baryon number at the electro-weak phase transition. It is clear that experimental studies of CP violation at RHIC are important and would enrich our understanding of bulk non-equilibrium dynamics in QCD and related gauge theories.

6.4. String theory: Calculations in the non-perturbative regime of physical quantities in field theories are generally very challenging. This is certainly the case for quantum chromodynamics. Reliable results from lattice QCD are currently limited to those obtained in thermal equilibrium, while sustained efforts to extend these methodologies to dynamical properties such as shear viscosity or collective modes are in their infancy. Recently, important progress made in an alternative approach to such calculations based on perturbative methods in a dual quantum field theory involving black holes in anti-de Sitter (AdS) space has attracted great interest, not only within the heavy ion community but also among string theorists. The new developments are based on the insight by Maldacena that weakly coupled string theories of gravity in higher dimensions are dual to a strongly-coupled gauge theory defined on the boundary of the space. It should be emphasized that these AdS/CFT (conformal field theory) techniques presently have the limitation that there is no known higher-dimensional gravity or string theory which is precisely dual to QCD. However, recent results in both the thermal sector and in the calculation of hadronic structure and scattering processes from applying these methods to QCD analogs suggest that the identification is quite general and may lead to new insights into strongly coupled gauge theories. 


\section{Summary}

The first five years have resulted in a consensus that a new state of rapidly thermalized matter is being created in heavy ion collisions at RHIC. There is evidence that the extremely hot and dense medium is a strongly coupled plasma that behaves as an almost perfect liquid. Estimates of the initial energy density at thermalization are $\sim 15$ times the transition energy density predicted by Lattice QCD, with the temperature at thermalization about twice the predicted transition temperature. There are also strong indications that gluon saturation effects in the colliding nuclei play an important role in the early stages of heavy ion collisions at RHIC - the earliest results are consistent with the Color Glass Condensate model, which describes a high energy density, saturated, highly coherent universal form of matter. Finally, as the world's first polarized proton collider, RHIC has begun the task of investigating the spin structure of the proton.

We have described in this report four fundamental questions that summarize the physics opportunities at RHIC in the next 10 years, and we have discussed in some detail how these questions might be addressed in a future physics program using $A+A, p(d)+A$ and polarized $p+p$ collisions. The heavy ion physics program will study the equation of state of the new matter, explore the QCD phase diagram, attempt to understand the strong coupling mechanisms that are in play, search for direct evidence of deconfinement, search for effects on hadron properties as chiral symmetry restoration is approached, study how the medium reaches thermal and chemical equilibration so rapidly, and study the mechanism by which the partonic medium converts to hadrons. The $\mathrm{p}(\mathrm{d})+\mathrm{A}$ program will probe the low momentum-fraction region of the nuclear wave function to characterize the initial conditions that lead to rapid thermalization in $\mathrm{A}+\mathrm{A}$ collisions and to determine the conditions under which the gluon density saturates. RHIC may be very well situated for studying gluon saturation because of the ability to effectively turn saturation effects on and off by varying collision species, collision energies and kinematic properties. The polarized proton program is now beginning to tackle an enormously ambitious set of measurements to explore the polarization of gluons, sea quarks and antiquarks in the proton, and to make transverse spin measurements intended to unravel transverse spin preferences from transverse motion preferences.

Most of the future physics programs at RHIC have been shown to depend crucially on the RHIC luminosity upgrade, and on the completion of the ongoing detector upgrades to PHENIX and STAR. In the case of the heavy ion program, the luminosity upgrade is critical to gaining access to far more powerful probes that involve inherently low cross sections. But it is also the key to opening up a large area of the QCD phase diagram to investigation at RHIC, by making it feasible to vary collision energy and colliding beam species many times in a single year - while still accumulating large integrated luminosities. For the polarized proton program, the factor of three increase in luminosity is essential for reaching the full potential of the sea quark polarization and transverse spin measurements, and will improve the precision of the gluon polarization measurements, which will already be well advanced by then.

Where relevant, we have commented on the relationship of the future program at RHIC II to the heavy ion physics program at the LHC, which will start up in the next few years. The field will be greatly enhanced by the advent of higher energy beams at the LHC, allowing measurements from the lowest RHIC energies to the top LHC energies, with many overlapping and many unique measurements at each. The initial temperature reached at the $\mathrm{LHC}$ will be about $4 \mathrm{~T}_{\mathrm{c}}$, instead of $2 \mathrm{~T}_{\mathrm{c}}$ at RHIC, raising the possibility of new physics phenomena being observed at the LHC. The jet 
yields and heavy quark cross sections will be much larger at the LHC because of the higher collision energy. On the other hand, the heavy quark yields at RHIC II will be similar to those at the LHC because of the higher luminosity and longer running times per year, while the initial conditions of temperature and heavy quark multiplicity will be so different that different quarkonium production mechanisms are predicted to dominate at the two facilities. Open heavy flavor energy loss can be studied at very different transverse momenta, very different energy densities of the medium, and possibly with significantly different energy loss mechanisms, making the programs strongly complementary. In the case of jet measurements, the much higher jet yields and higher transverse momentum reach at the LHC will enable studies of the medium interactions of much higher energy jets in a denser medium. Because of the much smaller background from multiple jets, RHIC will be very well suited to studies of lower momentum jets (which lose a large fraction of their energy) and of jet-medium recombination effects at intermediate $\mathrm{p}_{\mathrm{T}}$. Again, the programs will be strongly complementary. It must be emphasized that some very important aspects of the future physics program at RHIC are unique. The measurements at RHIC to study medium effects on the properties of hadrons described in this report are feasible at RHIC only because of the relatively small background from open charm. The exploration of the QCD phase diagram and search for the QCD critical point rely heavily on the collision energy range available at RHIC II, on the ability to rapidly change colliding beams, and on the electron cooling. And, of course, the RHIC polarized proton program is unique.

We have also tried to show the complementarity between the program at RHIC II to explore the QCD phase diagram to large baryon chemical potential and the lower energy fixed target program of the future FAIR facility, which will be constructed at GSI. RHIC II will explore the QCD phase diagram at higher temperature and in the range of baryonic chemical potential from $50-500 \mathrm{MeV}$, while FAIR will be able to access lower temperatures and the baryo-chemical potential range from 400-800 MeV. The two facilities will have strongly complementary programs studying in-medium properties of hadrons using low mass dilepton pairs, and searching for the QCD critical point.

Finally, we have briefly outlined ways in which the future physics program at RHIC connects to the study of $\mathrm{CP}$ violation and the fields of plasma physics, condensed matter physics, cosmology and string theory. 


\section{Appendix 1}

\section{RHIC II science workshops}

This series of workshops began in November 2004, at which time seven Working Groups were initiated. These groups met in workshops through 2005, with the purpose of providing an organized forum for the community to address and describe quantitatively the most important science issues for the proposed RHIC II luminosity upgrade, and corresponding detector upgrades. Each Working Group was led by three convenors representing theory and experiment.

The organization of the workshops is listed below. Each of the Working Groups has produced a detailed report (except for the "New Directions" group, which provided a sounding board and input to the other groups). The Working Group reports can be found at www.bnl.gov/physics/rhicIIscience. This summary document is based on these reports, and was prepared by a Writing Committee that included at least one convenor from each of the Working Groups.

\section{Writing committee:}

Mark Baker, Tony Frawley (co-Chair), Carl Gagliardi (co-Chair), Ulrich Heinz, Matthias GrossePerdekamp, Tim Hallman, John Harris, Larry McLerran, Saskia Mioduszewski, Berndt Mueller, Jamie Nagle, Julia Velkovska, Steve Vigdor, Werner Vogelsang, Xin-Nian Wang, Zhangbu Xu, Bill Zajc

\section{Workshop organization:}

Organizers: Sam Aronson, Tom Ludlam

Steering Committee: U. Heinz (Ohio State), B. Jacak (Stony Brook), D. Kharzeev (BNL), D. Morrison (BNL), G. Roland (MIT), E. Shuryak (Stony Brook), T. Ullrich (BNL), W. Vogelsang (BNL), Z. Xu (BNL)

\section{Working Group convenors:}

Electromagnetic Probes: Gabor David, Ralf Rapp, Zhangbu Xu

Heavy Flavor: Tony Frawley, Thomas Ullrich, Ramona Vogt

High $P_{T}$ : Kirill Filimonov, Saskia Mioduszewski, Denes Molnar

Equation of State: Steffen Bass, Helen Caines, Julia Velkovska

Forward Physics and pA: Carl Gagliardi, Mike Leitch, Kirill Tuchin

Spin and pp: Matthias Grosse-Perdekamp, Enst Sichtermann, Marco Stratmann

New Directions: Berndt Mueller, Jamie Nagle, Peter Steinberg 